Publ. RIMS, Kyoto Univ.

41 (2005), 599-681

\title{
Higher Arithmetic $K$-Theory
}

By

\author{
Yuichiro TAKEDA*
}

\begin{abstract}
A concrete definition of higher $K$-theory in Arakelov geometry is given. The $K$-theory defined in this paper is a higher extension of the arithmetic $K_{0}$-group of an arithmetic variety defined by Gillet and Soulé. Products and direct images in this $K$-theory are discussed.
\end{abstract}

\section{$\S 1 . \quad$ Introduction}

The aim of this paper is to provide a new definition of higher $K$-theory in Arakelov geometry and to show that it enjoys the same formal properties as the higher algebraic $K$-theory of schemes.

Let $X$ be a proper arithmetic variety, namely, a regular scheme which is flat and proper over $\mathbb{Z}$, the ring of integers. In the research on the arithmetic Chern character of a hermitian vector bundle on $X$, Gillet and Soule defined the arithmetic $K_{0}$-group $\widehat{K}_{0}(X)$ of $X[9]$. It can be viewed as an analogue in Arakelov geometry of the $K_{0}$-group of vector bundles on a scheme.

After the advent of $\widehat{K}_{0}(X)$, its higher extension was discussed in $[6,7,14]$. In these papers one common thing was suggested that higher arithmetic $K$ theory should be obtained as the homotopy group of the homotopy fiber of the Beilinson's regulator map. To be more precise, there should exist a group $K M_{n}(X)$ for each $n \geq 0$ fitting into the long exact sequence

$$
\cdots \rightarrow K_{n+1}(X) \stackrel{\rho}{\rightarrow} \underset{p}{\oplus} H_{\mathcal{D}}^{2 p-n-1}(X, \mathbb{R}(p)) \rightarrow K M_{n}(X) \rightarrow K_{n}(X) \rightarrow \cdots,
$$

Communicated by S. Mochizuki. Received April 25, 2003. Revised June 10, 2004, August 10, 2004.

2000 Mathematics Subject Classification(s): Primary 14G40; Secondary 19E08.

*Faculty of Mathematics, Kyushu University, 6-10-1 Hakozaki, Higashi-Ku, Fukuoka, 8128581, Japan.

e-mail: yutakeda@math.kyushu-u.ac.jp

(C) 2005 Research Institute for Mathematical Sciences, Kyoto University. All rights reserved. 
where $H_{\mathcal{D}}^{n}(X, \mathbb{R}(p))$ is the real Deligne cohomology of $X$ and $\rho$ is the Beilinson's regulator map.

To get the homotopy fiber, a simplicial description of the regulator map is necessary. And it has already been given by Burgos and Wang in [6]. For a compact complex manifold $M$, they introduced an exact cube of hermitian vector bundles on $M$ and associated with it a differential form called a higher Bott-Chern form. This gives a homomorphism of complexes

$$
\operatorname{ch}: \mathbb{Z} \widehat{S}_{*}(M) \rightarrow \mathcal{D}^{*}(M, p)[2 p+1]
$$

from the homology complex $\mathbb{Z} \widehat{S}_{*}(M)$ of the S-construction of the category of hermitian vector bundles on $M$ to the complex $\mathcal{D}^{*}(M, p)$ computing the real Deligne cohomology of $M$ defined in [4]. It is the main theorem of [6] that the following map coincides with the Beilinson's regulator map:

$$
\rho: K_{n}(M) \simeq \pi_{n+1}(\widehat{S}(M)) \stackrel{\text { Hurewicz }}{\longrightarrow} H_{n+1}\left(\mathbb{Z} \widehat{S}_{*}(M)\right) \stackrel{H(\mathrm{ch})}{\longrightarrow} H_{\mathcal{D}}^{2 p-n}(M, \mathbb{R}(p)) .
$$

Applying this to the complex manifold $X(\mathbb{C})$ associated with $X$, we can obtain a simplicial description of the regulator map for $X$.

In this paper, we will give another definition of higher arithmetic $K$-theory for a proper arithmetic variety. One of remarkable features of our arithmetic $K$-theory is that it is given as an extension of the algebraic $K$-theory by the cokernel of the regulator map.

Before explaining our method, let us recall the definition of $\widehat{K}_{0}(X)$ by Gillet and Soulé [9]. For a proper arithmetic variety $X$, let $\mathcal{A}^{p, p}(X)$ be the space of real $(p, p)$-forms $\omega$ on $X(\mathbb{C})$ such that $\bar{F}_{\infty}^{*} \omega=(-1)^{p} \omega$ for the complex conjugation $F_{\infty}$ on $X(\mathbb{C})$ and let $\widetilde{\mathcal{A}}(X)=\oplus_{p} \mathcal{A}^{p, p}(X) /(\operatorname{Im} \partial+\operatorname{Im} \bar{\partial})$. Then $\widehat{K}_{0}(X)$ is defined as a factor group of the free abelian group generated by pairs $(\bar{E}, \omega)$ where $\bar{E}$ is a hermitian vector bundle on $X$ and $\omega \in \widetilde{\mathcal{A}}(X)$. Relations on pairs are given by each short exact sequence $\mathcal{E}: 0 \rightarrow \overline{E^{\prime}} \rightarrow \bar{E} \rightarrow \overline{E^{\prime \prime}} \rightarrow 0$ and $\omega^{\prime}, \omega^{\prime \prime} \in \widetilde{\mathcal{A}}(X)$ as follows:

$$
\left(\overline{E^{\prime}}, \omega^{\prime}\right)+\left(\overline{E^{\prime \prime}}, \omega^{\prime \prime}\right)=\left(\bar{E}, \omega^{\prime}+\omega^{\prime \prime}+\widetilde{c h}(\mathcal{E})\right),
$$

where $\widetilde{\operatorname{ch}}(\mathcal{E})$ is the Bott-Chern secondary characteristic class of $\mathcal{E}$.

The above definition of $\widehat{K}_{0}(X)$ can be rephrased in terms of loops and homotopies on $|\widehat{S}(X)|$, the topological realization of the $S$-construction of the category of hermitian vector bundles on $X$. Consider a pair $(l, \omega)$, where $l$ is a pointed simplicial loop on $|\widehat{S}(X)|$ and $\omega \in \widetilde{\mathcal{A}}(X)$. Two pairs $(l, \omega)$ and $\left(l^{\prime}, \omega^{\prime}\right)$ are said to be homotopy equivalent if there is a cellular homotopy $H$ : $\left(S^{1} \times I\right) /(\{*\} \times I) \rightarrow|\widehat{S}(X)|$ from $l$ to $l^{\prime}$ such that the Bott-Chern secondary 
characteristic class $\widetilde{\operatorname{ch}}(H)$ of $H$, which is defined in a natural way, is equal to $\omega^{\prime}-\omega$. Let $\widehat{\pi}_{1}(|\widehat{S}(X)|, \widetilde{c h})$ denote the set of all equivalence classes of such pairs. Then it carries the structure of an abelian group and the map

$$
\widehat{K}_{0}(X) \rightarrow \widehat{\pi}_{1}(|\widehat{S}(X)|, \tilde{\mathrm{ch}})
$$

given by $(\bar{E}, \omega) \mapsto\left(l_{\bar{E}},-\omega\right)$, where $l_{\bar{E}}$ is the simplicial loop on $|\widehat{S}(X)|$ determined by $\bar{E}$, is proved to be bijective.

Let us generalize this observation to higher homotopy groups in a general setting. Take a pointed CW-complex $T$ and a homomorphism

$$
\rho: C_{*}(T) \rightarrow W_{*}
$$

from the reduced homology complex of $T$ to a chain complex of abelian groups $W_{*}$. Let $S^{n}$ denote the $n$-dimensional sphere and consider a pair $(f, \omega)$ of a pointed cellular map $f: S^{n} \rightarrow T$ and $\omega \in \widetilde{W}_{n}=W_{n} / \operatorname{Im} \partial$. Two pairs $(f, \omega)$ and $\left(f^{\prime}, \omega^{\prime}\right)$ are said to be homotopy equivalent if there is a pointed cellular homotopy $H:\left(S^{n} \times I\right) /(\{*\} \times I) \rightarrow T$ from $f$ to $f^{\prime}$ such that the image of the fundamental chain of $H$ by $\rho$ is equal to $(-1)^{n+1}\left(\omega^{\prime}-\omega\right)$. This actually gives an equivalence relation on the set of such pairs. The set of homotopy equivalence classes has the structure of a group and it becomes an abelian group when $n \geq 2$. This group is denoted by $\widehat{\pi}_{n}(T, \rho)$ and called the $n$-th homotopy group of $T$ modified by $\rho$. We define the $n$-th arithmetic $K$-theory of a proper arithmetic variety $X$ as the $(n+1)$-th homotopy group of $|\widehat{S}(X)|$ modified by the higher Bott-Chern form:

$$
\widehat{K}_{n}(X)=\widehat{\pi}_{n+1}(|\widehat{S}(X)|, \mathrm{ch}) .
$$

We will show that $\widehat{K}_{n}(X)$ possesses the same properties as the usual higher $K$-theory of schemes. More precisely, we will show the following:

(1) Fundamental exact sequence:

$$
K_{n+1}(X) \rightarrow \widetilde{\mathcal{D}}_{n+1}(X) \rightarrow \widehat{K}_{n}(X) \rightarrow K_{n}(X) \rightarrow 0,
$$

where $\widetilde{\mathcal{D}}_{n+1}(X)=\mathcal{D}_{n+1}(X) / \operatorname{Im} d_{\mathcal{D}}$. In the case of $\widehat{K}_{0}(X)$, this exact sequence has been obtained in [9].

(2) Chern class map:

$$
\operatorname{ch}_{n}: \widehat{K}_{n}(X) \rightarrow \mathcal{D}_{n}(X) .
$$

If we denote $K M_{n}(X)=\operatorname{Ker}_{n}$, then we can obtain the long exact sequence

$$
\cdots \rightarrow K_{n+1}(X) \stackrel{\rho}{\rightarrow} \underset{p}{\oplus} H_{\mathcal{D}}^{2 p-n-1}(X, \mathbb{R}(p)) \rightarrow K M_{n}(X) \rightarrow K_{n}(X) \stackrel{\rho}{\rightarrow} \cdots
$$


We show that $K M_{n}(X)$ is canonically isomorphic to the homotopy fiber of the Bott-Chern form.

(3) Arakelov $K$-theory: Fix an $F_{\infty}$-invariant Kähler metric $h_{X}$ on $X(\mathbb{C})$. The pair $\bar{X}=\left(X, h_{X}\right)$ is called an Arakelov variety. We can define Arakelov $K$-group of $\bar{X}$ as

$K_{n}(\bar{X})=\left\{x \in \widehat{K}_{n}(X) ; \operatorname{ch}_{n}(x)\right.$ is a harmonic form with respect to $\left.h_{X}\right\}$.

We have the exact sequence

$$
K_{n+1}(X) \stackrel{\rho}{\rightarrow} \underset{p}{\oplus} H_{\mathcal{D}}^{2 p-n-1}(X, \mathbb{R}(p)) \rightarrow K_{n}(\bar{X}) \rightarrow K_{n}(X) \rightarrow 0 .
$$

(4) Products: $\widehat{K}_{*}(X)$ has a product

$$
\widehat{K}_{n}(X) \times \widehat{K}_{m}(X) \rightarrow \widehat{K}_{n+m}(X) .
$$

It does not admit the associative law. But if we restrict this to $K_{*}(\bar{X})$, it becomes associative. It is shown that the product is graded commutative up to 2-torsion.

(5) Functoriality: For arbitrary morphism $f: X \rightarrow Y$, we can define pull back map

$$
\widehat{f}^{*}: \widehat{K}_{n}(Y) \rightarrow \widehat{K}_{n}(X) .
$$

It is compatible with product. Suppose that $f$ is smooth and projective, and fix a Kähler metric on the relative tangent bundle of $f(\mathbb{C}): X(\mathbb{C}) \rightarrow$ $Y(\mathbb{C})$. Then we can define direct image homomorphism

$$
\widehat{f}_{*}: \widehat{K}_{n}(X) \rightarrow \widehat{K}_{n}(Y) .
$$

The projection formula for $\widehat{f}_{*}$ and $\widehat{f}^{*}$ holds.

From the above properties, we can obtain a non-canonical decomposition of $\widehat{K}_{n}(X)$ into three summands:

$$
\widehat{K}_{n}(X) \simeq K_{n}(X) \oplus\left(\mathcal{D}_{n+1}(X) / \operatorname{Ker} d_{\mathcal{D}}\right) \oplus\left({\underset{p}{p}}_{\mathcal{D}}^{2 p-n-1}(X, \mathbb{R}(p)) / \operatorname{Im} \rho\right) .
$$

The Bass' conjecture says that the first summand is a finitely generated abelian group. The second one is an infinite dimensional $\mathbb{R}$-vector space, and the Beilinson's conjectures imply that the third one becomes a real torus.

Let us describe the organization of the paper: In $\S 2$ we introduce some materials used in the paper, such as $S$-construction, exact cubes and higher 
Bott-Chern forms. In $\S 3$ we propose the notion of modified homotopy groups. In $\S 4$ we give the definition of the higher arithmetic $K$-group $\widehat{K}_{*}(X)$ and deduce the fundamental exact sequence. We also define the Arakelov $K$-group. In $\S 5$ we prove a product formula for higher Bott-Chern forms. It provides an alternative proof of the fact that the regulator map respects the products. In $\S 6$, we discuss product in higher arithmetic $K$-theory. In $\S 7$, we define a direct image homomorphism in higher arithmetic $K$-theory. To do this we employ the higher analytic torsion form of an exact hermitian cube defined by Roessler [13]. Moreover we establish the projection formula.

\section{§2. Preliminaries}

\section{§2.1. Conventions on complexes}

Let us first settle some conventions on complexes. By complex of an abelian category $\mathfrak{A}$, we mean a family of objects $\left\{A^{k}\right\}_{k \in \mathbb{Z}}$ with differential $d_{A}: A^{k} \rightarrow$ $A^{k+1}$. For a complex $A^{*}$ and $n \in \mathbb{Z}$, the $n$-th translation $A[n]^{*}$ is defined as $A[n]^{k}=A^{n+k}$ and $d_{A[n]}=(-1)^{n} d_{A}$.

By chain complex we mean a family of objects $\left\{A_{k}\right\}_{k \geq 0}$ with boundary $\partial_{A}: A_{k} \rightarrow A_{k-1}$. For a complex $A^{*}=\left(A^{k}, d_{A}\right)$ such that $A^{k}=0$ for $k>0$, we can define a chain complex $A_{*}$ as $A_{k}=A^{-k}$ and $d_{A}=\partial_{A}$. The $n$-th translation $A[n]_{*}$ of a chain complex $A_{*}$ for $n \geq 0$ is defined as $A[n]_{k}=A_{k-n}$ and $\partial_{A[n]}=(-1)^{n} \partial_{A}$.

\section{$\S 2.2 . \quad S$-construction}

In this subsection we recall the $S$-construction developed by Waldhausen [15]. Throughout this paper, we assume that any small exact category has a distinguished zero object denoted by 0 . Let $[n]$ be the finite ordered set $\{0,1, \ldots, n\}$ and $\operatorname{Ar}[n]$ the category of arrows of $[n]$. For a small exact category $\mathfrak{A}$, let $S_{n} \mathfrak{A}$ be the set of functors $E: \operatorname{Ar}[n] \rightarrow \mathfrak{A}$ satisfying the following conditions for $E_{i, j}=E(i \leq j)$ :

(1) $E_{i, i}=0$ for any $0 \leq i \leq n$.

(2) For any $i \leq j \leq k, E_{i, j} \rightarrow E_{i, k} \rightarrow E_{j, k}$ is a short exact sequence of $\mathfrak{A}$.

For example, $S_{0} \mathfrak{A}=\{0\}, S_{1} \mathfrak{A}$ is the set of objects of $\mathfrak{A}$ and $S_{2} \mathfrak{A}$ is the set of short exact sequences of $\mathfrak{A}$. The functor $S \mathfrak{A}:[n] \mapsto S_{n} \mathfrak{A}$ becomes a simplicial set with the base point given by $0 \in S_{0} \mathfrak{A}$. It is shown in [15] that $S \mathfrak{A}$ is homotopy equivalent to the Quillen's Q-construction of $\mathfrak{A}$. Therefore the $(n+$ 
1)-th homotopy group $\pi_{n+1}(S \mathfrak{A}, 0)$ is isomorphic to $K_{i}(\mathfrak{A})$, the algebraic $K$ theory of $\mathfrak{A}$.

\section{§2.3. $\quad$ Exact $n$-cubes}

Let us recall the notion of an exact $n$-cube. For more details, see $[5,6]$. Let $\langle-1,0,1\rangle$ be the ordered set consisting of three elements. An $n$-cube of a small exact category $\mathfrak{A}$ is a covariant functor from the $n$-th power of $\langle-1,0,1\rangle$ to $\mathfrak{A}$. For an $n$-cube $\mathcal{F}$, we denote by $\mathcal{F}_{\alpha_{1}, \ldots, \alpha_{n}}$ the image of an object $\left(\alpha_{1}, \ldots, \alpha_{n}\right)$ of $\langle-1,0,1\rangle^{n}$. For integers $i$ and $j$ satisfying $1 \leq i \leq$ $n$ and $-1 \leq j \leq 1$, an $(n-1)$-cube $\partial_{i}^{j} \mathcal{F}$ is given by $\left(\partial_{i}^{j} \mathcal{F}\right)_{\alpha_{1}, \ldots, \alpha_{n-1}}=$ $\mathcal{F}_{\alpha_{1}, \ldots, \alpha_{i-1}, j, \alpha_{i}, \ldots, \alpha_{n-1}}$. It is called a face of $\mathcal{F}$. For an object $\alpha$ of $\langle-1,0$, $1\rangle^{n-1}$ and an integer $i$ satisfying $1 \leq i \leq n$, a 1-cube $\partial_{i^{c}}^{\alpha} \mathcal{F}$ called an edge of $\mathcal{F}$ is

$$
\mathcal{F}_{\alpha_{1}, \ldots, \alpha_{i-1},-1, \alpha_{i}, \ldots, \alpha_{n-1}} \rightarrow \mathcal{F}_{\alpha_{1}, \ldots, \alpha_{i-1}, 0, \alpha_{i}, \ldots, \alpha_{n-1}} \rightarrow \mathcal{F}_{\alpha_{1}, \ldots, \alpha_{i-1}, 1, \alpha_{i}, \ldots, \alpha_{n-1}} .
$$

An $n$-cube $\mathcal{F}$ is said to be exact if all edges of $\mathcal{F}$ are short exact sequences.

Let $C_{n} \mathfrak{A}$ denote the set of all exact $n$-cubes of $\mathfrak{A}$. If $\mathcal{F}$ is an exact $n$-cube, then any face $\partial_{i}^{j} \mathcal{F}$ is also exact. Hence $\partial_{i}^{j}$ induces a map

$$
\partial_{i}^{j}: C_{n} \mathfrak{A} \rightarrow C_{n-1} \mathfrak{A} .
$$

Let $\mathcal{F}$ be an exact $n$-cube of $\mathfrak{A}$. For an integer $i$ satisfying $1 \leq i \leq n+1$, let $s_{i}^{1} \mathcal{F}$ be an exact $(n+1)$-cube such that its edge $\partial_{i^{c}}^{\alpha}\left(s_{i}^{1} \mathcal{F}\right)$ is $\mathcal{F}_{\alpha} \stackrel{\text { id }}{\rightarrow} \mathcal{F}_{\alpha} \rightarrow 0$. Similarly, let $s_{i}^{-1} \mathcal{F}$ be an exact $(n+1)$-cube such that $\partial_{i^{c}}^{\alpha}\left(s_{i}^{-1} \mathcal{F}\right)$ is $0 \rightarrow \mathcal{F}_{\alpha} \stackrel{\text { id }}{\rightarrow}$ $\mathcal{F}_{\alpha}$. An exact cube written as $s_{i}^{j} \mathcal{F}$ is said to be degenerate.

Let $\mathbb{Z} C_{n} \mathfrak{A}$ be the free abelian group generated by $C_{n} \mathfrak{A}$ and $D_{n} \subset \mathbb{Z} C_{n} \mathfrak{A}$ the subgroup generated by all degenerate exact $n$-cubes. Let $\widetilde{\mathbb{Z}} C_{n} \mathfrak{A}=\mathbb{Z} C_{n} \mathfrak{A} / D_{n}$ and

$$
\partial=\sum_{i=1}^{n} \sum_{j=-1}^{1}(-1)^{i+j+1} \partial_{i}^{j}: \widetilde{\mathbb{Z}} C_{n} \mathfrak{A} \rightarrow \widetilde{\mathbb{Z}} C_{n-1} \mathfrak{A} .
$$

Then $\widetilde{\mathbb{Z}} C_{*} \mathfrak{A}=\left(\widetilde{\mathbb{Z}} C_{n} \mathfrak{A}, \partial\right)$ becomes a chain complex.

In $[6, \S 4.4]$, an exact $(n-1)$-cube $\operatorname{Cub}(E)$ for any $E \in S_{n} \mathfrak{A}$ is constructed and it is shown that $E \mapsto \operatorname{Cub}(E)$ induces a homomorphism of complexes

$$
\mathrm{Cub}: \mathbb{Z} S_{*} \mathfrak{A}[1] \rightarrow \widetilde{\mathbb{Z}} C_{*} \mathfrak{A} .
$$




\section{§2.4. Higher Bott-Chern forms}

In this subsection we recall higher Bott-Chern forms developed by Burgos and Wang. For more details, see [5,6]. First we introduce the recipient of higher Bott-Chern forms. Let $M$ be a compact complex algebraic manifold, namely, the analytic space consisting of all $\mathbb{C}$-valued points of a smooth proper algebraic variety over $\mathbb{C}$. Let $\mathcal{E}_{\mathbb{R}}^{p}(M)$ be the space of real smooth differential forms of degree $p$ on $M$ and $\mathcal{E}^{p}(M)=\mathcal{E}_{\mathbb{R}}^{p}(M) \otimes_{\mathbb{R}} \mathbb{C}$. Let $\mathcal{E}^{p, q}(M)$ be the space of complex differential forms of type $(p, q)$ on $M$. Set

$$
\mathcal{D}^{n}(M, p)= \begin{cases}\mathcal{E}_{\mathbb{R}}^{n-1}(M)(p-1) \cap \underset{\substack{p^{\prime}+q^{\prime}=n-1 \\ p^{\prime}<p, q^{\prime}<p}}{\oplus \mathcal{E}^{p^{\prime}, q^{\prime}}(M),} & n<2 p, \\ \mathcal{E}_{\mathbb{R}}^{2 p}(M)(p) \cap \mathcal{E}^{p, p}(M) \cap \operatorname{Ker} d, & n=2 p, \\ 0, & n>2 p\end{cases}
$$

and define a differential $d_{\mathcal{D}}: \mathcal{D}^{n}(M, p) \rightarrow \mathcal{D}^{n+1}(M, p)$ by

$$
d_{\mathcal{D}}(\omega)= \begin{cases}-\pi(d \omega), & n<2 p-1 \\ -2 \partial \bar{\partial} \omega, & n=2 p-1 \\ 0, & n>2 p-1\end{cases}
$$

where $\pi: \mathcal{E}^{n}(M) \rightarrow \mathcal{D}^{n}(M, p)$ is the canonical projection. Then it is shown in $\left[4\right.$, Thm. 2.6] that the pair $\left(\mathcal{D}^{*}(M, p), d_{\mathcal{D}}\right)$ is a complex of $\mathbb{R}$-vector spaces with

$$
H^{n}\left(\mathcal{D}^{*}(M, p), d_{\mathcal{D}}\right) \simeq H_{\mathcal{D}}^{n}(M, \mathbb{R}(p))
$$

for $n \leq 2 p$.

By a hermitian vector bundle $\bar{E}=(E, h)$ on $M$ we mean an algebraic vector bundle $E$ on $M$ with a smooth hermitian metric $h$. Let $K_{\bar{E}}$ denote the curvature form of the unique connection on $\bar{E}$ that is compatible with both the metric and the complex structure. Let us write

$$
\operatorname{ch}_{0}(\bar{E})=\operatorname{Tr}\left(\exp \left(-K_{\bar{E}}\right)\right) \in \underset{p}{\oplus} \mathcal{D}^{2 p}(M, p) .
$$

An exact hermitian $n$-cube on $M$ is an exact $n$-cube made of hermitian vector bundles on $M$. Let $\mathcal{F}=\left\{\bar{E}_{\alpha}\right\}$ be an exact hermitian $n$-cube on $M$. We call $\mathcal{F}$ an emi-n-cube if the metric on any $\bar{E}_{\alpha}$ with $\alpha_{i}=1$ coincides with the metric induced from $\bar{E}_{\alpha_{1}, \ldots, \alpha_{i-1}, 0, \alpha_{i+1}, \ldots, \alpha_{n}}$ for the surjection $\bar{E}_{\alpha_{1}, \ldots, \alpha_{i-1}, 0, \alpha_{i+1}, \ldots, \alpha_{n}} \rightarrow \bar{E}_{\alpha}$.

For an emi-1-cube $\mathcal{E}: \bar{E}_{-1} \rightarrow \bar{E}_{0} \rightarrow \bar{E}_{1}$, a canonical way of constructing a hermitian vector bundle $\operatorname{tr}_{1} \mathcal{E}$ on $M \times \mathbb{P}^{1}$ connecting $\bar{E}_{0}$ with $\bar{E}_{-1} \oplus \bar{E}_{1}$ is 
given in [6]. More precisely, if $(x: y)$ denotes the homogeneous coordinate of $\mathbb{P}^{1}$ and $z=x / y$, then $\operatorname{tr}_{1} \mathcal{E}$ is a hermitian vector bundle on $M \times \mathbb{P}^{1}$ satisfying the following conditions:

$$
\left.\operatorname{tr}_{1} \varepsilon\right|_{z=0} \simeq \bar{E}_{0},\left.\operatorname{tr}_{1} \varepsilon\right|_{z=\infty} \simeq \bar{E}_{-1} \oplus \bar{E}_{1}
$$

For an emi- $n$-cube $\mathcal{F}$, let $\operatorname{tr}_{1}(\mathcal{F})$ be an emi- $(n-1)$-cube on $M \times \mathbb{P}^{1}$ given by $\operatorname{tr}_{1}(\mathcal{F})_{\alpha}=\operatorname{tr}_{1}\left(\partial_{n^{c}}^{\alpha}(\mathcal{F})\right)$ for $\alpha \in\langle-1,0,1\rangle^{n-1}$, and $\operatorname{tr}_{n}(\mathcal{F})$ a hermitian vector bundle on $M \times\left(\mathbb{P}^{1}\right)^{n}$ given by

$$
\operatorname{tr}_{n}(\mathcal{F})=\overbrace{\operatorname{tr}_{1} \operatorname{tr}_{1} \ldots \operatorname{tr}_{1}}^{n \text { times }}(\mathcal{F}) .
$$

Let $\pi_{i}:\left(\mathbb{P}^{1}\right)^{n} \rightarrow \mathbb{P}^{1}$ be the $i$-th projection and $z_{i}=\pi_{i}^{*} z$. For an integer $i$ satisfying $1 \leq i \leq n$,

$$
S_{n}^{i}=\sum_{\sigma \in \mathfrak{S}_{n}}(-1)^{\sigma} \log \left|z_{\sigma(1)}\right|^{2} \frac{d z_{\sigma(2)}}{z_{\sigma(2)}} \wedge \cdots \wedge \frac{d z_{\sigma(i)}}{z_{\sigma(i)}} \wedge \frac{d \bar{z}_{\sigma(i+1)}}{\bar{z}_{\sigma(i+1)}} \wedge \cdots \wedge \frac{d \bar{z}_{\sigma(n)}}{\bar{z}_{\sigma(n)}},
$$

which is a differential form with logarithmic poles on $\left(\mathbb{P}^{1}\right)^{n}$. The Bott-Chern form of an emi- $n$-cube $\mathcal{F}$ is

$$
\operatorname{ch}_{n}(\mathcal{F})=\frac{1}{(2 \pi \sqrt{-1})^{n}} \int_{\left(\mathbb{P}^{1}\right)^{n}} \operatorname{ch}_{0}\left(\operatorname{tr}_{n}(\mathcal{F})\right) \wedge T_{n} \in \underset{p}{\oplus} \mathcal{D}^{2 p-n}(M, p),
$$

where

$$
T_{n}=\frac{(-1)^{n}}{2 n !} \sum_{i=1}^{n}(-1)^{i} S_{n}^{i} .
$$

A process to produce an emi- $n$-cube $\lambda \mathcal{F}$ from an arbitrary exact hermitian $n$-cube $\mathcal{F}$ is given in [6]. By virtue of this process, we can extend the definition of the Bott-Chern form to an arbitrary exact hermitian $n$-cube.

Definition 2.1. The Bott-Chern form of an exact hermitian $n$-cube $\mathcal{F}$ is an element of $\oplus_{p} \mathcal{D}^{2 p-n}(M, p)$ given as follows:

$$
\operatorname{ch}_{n}(\mathcal{F})=\frac{1}{(2 \pi \sqrt{-1})^{n}} \int_{\left(\mathbb{P}^{1}\right)^{n}} \operatorname{ch}_{0}\left(\operatorname{tr}_{n}(\lambda \mathcal{F})\right) \wedge T_{n}
$$

Theorem $2.2([6])$. Let $\widehat{\mathcal{P}}(M)$ denote the category of hermitian vector bundles on $M$ and let $\widetilde{\mathbb{Z}} \widehat{C}_{*}(M)=\widetilde{\mathbb{Z}} C_{*} \widehat{\mathcal{P}}(M)$. Then $\mathcal{F} \mapsto \operatorname{ch}_{n}(\mathcal{F})$ induces a homomorphism

$$
\operatorname{ch}: \widetilde{\mathbb{Z}} \widehat{C}_{*}(M) \rightarrow \underset{p}{\oplus} \mathcal{D}^{*}(M, p)[2 p] .
$$


Moreover, the following map

$$
\begin{aligned}
K_{n}(M)= & \pi_{n+1}(\widehat{S}(M)) \stackrel{\text { Hurewicz }}{\longrightarrow} H_{n+1}\left(\mathbb{Z} \widehat{S}_{*}(M)\right) \\
& \stackrel{\text { Cub }}{\longrightarrow} H_{n}\left(\widetilde{\mathbb{Z}} \widehat{C}_{*}(M)\right) \stackrel{\text { ch }}{\longrightarrow} \underset{p}{\oplus} H_{\mathcal{D}}^{2 p-n}(M, \mathbb{R}(p))
\end{aligned}
$$

coincides with the Beilinson's regulator map.

\section{$\S 3 . \quad$ Modified Homotopy Groups}

\section{§3.1. Definition of modified homotopy groups}

In this section we develop a general framework used later in this paper. Let $I$ be the closed interval $[0,1]$ equipped with the usual CW-complex structure. Throughout this paper we identify the $n$-dimensional sphere $S^{n}$ with $I^{n} / \partial I^{n}$. Therefore $S^{n}$ consists of two cells and any point of $S^{n}$ except the base point is expressed by an $n$-tuple of real numbers $\left(t_{1}, \ldots, t_{n}\right)$ with $0<t_{i}<1$.

Let $T$ be a pointed $\mathrm{CW}$-complex and $* \in T$ the base point. Let $\operatorname{sk}_{n}(T)$ be the $n$-th skeleton of $T$ when $n \geq 0$ and $\operatorname{sk}_{-1}(T)=\{*\}$. For $n \geq 0$, let us write $C_{n}(T)=H_{n}\left(\operatorname{sk}_{n}(T), \mathrm{sk}_{n-1}(T) ; \mathbb{Z}\right)$, the $n$-th relative homology group of the pair $\left(\operatorname{sk}_{n}(T), \mathrm{sk}_{n-1}(T)\right)$. Let $\partial: C_{n}(T) \rightarrow C_{n-1}(T)$ be the connecting homomorphism for the triple $\left(\operatorname{sk}_{n}(T), \mathrm{sk}_{n-1}(T), \mathrm{sk}_{n-2}(T)\right)$. Then $\left(C_{*}(T), \partial\right)$ is a chain complex whose homology group is isomorphic to the reduced homology group of $T$.

Suppose that a chain complex of abelian groups $\left(W_{*}, \partial\right)$ and a homomorphism of chain complexes $\rho: C_{*}(T) \rightarrow W_{*}$ are given. Let $\widetilde{W}_{n}=W_{n} / \operatorname{Im} \partial$. Let us consider a pair $(f, \omega)$ of a pointed cellular map $f: S^{n} \rightarrow T$ and $\omega \in \widetilde{W}_{n+1}$. A cellular homotopy from one pair $(f, \omega)$ to another pair $\left(f^{\prime}, \omega^{\prime}\right)$ is a pointed cellular map $H:\left(S^{n} \times I\right) /(\{*\} \times I) \rightarrow T$ satisfying the following:

(1) $H(x, 0)=f(x)$ and $H(x, 1)=f^{\prime}(x)$.

(2) Let $\left[S^{n} \times I\right] \in C_{n+1}\left(S^{n} \times I\right)$ denote the fundamental chain of $S^{n} \times I$, where the orientation on $S^{n} \times I$ is inherited from the canonical orientation of the interval $I$. Then

$$
\omega^{\prime}-\omega=(-1)^{n+1} \rho H_{*}\left(\left[S^{n} \times I\right]\right) .
$$

It can be shown that the cellular homotopy gives an equivalence relation on the set of pairs. Two pairs are said to be homotopy equivalent if there exists a cellular homotopy between them. We denote by $\widehat{\pi}_{n}(T, \rho)$ the set of all homotopy equivalence classes of pairs. 
Let us define a multiplication on the set $\widehat{\pi}_{n}(T, \rho)$. Let $T \vee T=\{(x, y) \in$ $T \times T ; x=*$ or $y=*\}$. Then we can define a natural map $T \vee T \rightarrow T$ by $(x, *) \mapsto x$ and $(*, y) \mapsto y$. A comultiplication map $\mu: S^{n} \rightarrow S^{n} \vee S^{n}$ is given by

$$
\mu\left(t_{1}, \ldots, t_{n}\right)= \begin{cases}\left(\left(t_{1}, t_{2}, \ldots, 2 t_{n}\right), *\right), & 0<t_{n} \leq \frac{1}{2}, \\ \left(*,\left(t_{1}, t_{2}, \ldots, 2 t_{n}-1\right)\right), & \frac{1}{2} \leq t_{n}<1\end{cases}
$$

and a homotopy inverse map $\nu: S^{n} \rightarrow S^{n}$ by $\nu\left(t_{1}, \ldots, t_{n-1}, t_{n}\right)=\left(t_{1}, \ldots, t_{n-1}\right.$, $\left.1-t_{n}\right)$. For two pointed cellular maps $f, g: S^{n} \rightarrow T$, let us write

$$
f \cdot g: S^{n} \stackrel{\mu}{\longrightarrow} S^{n} \vee S^{n} \stackrel{f \vee g}{\longrightarrow} T \vee T \rightarrow T,
$$

and

$$
f^{-1}: S^{n} \stackrel{\nu}{\rightarrow} S^{n} \stackrel{f}{\rightarrow} T .
$$

A multiplication of two pairs $(f, \omega)$ and $(g, \tau)$ is

$$
(f, \omega) \cdot(g, \tau)=(f \cdot g, \omega+\tau) .
$$

It is easy to show that the multiplication - is compatible with the homotopy equivalence relation on pairs. Hence it gives rise to a multiplication on $\widehat{\pi}_{n}(T, \rho)$.

Let us next verify the associativity of the multiplication. For three pointed cellular maps $f, g, h: S^{n} \rightarrow T$, a cellular homotopy $H_{1}:\left(S^{n} \times I\right) /(\{*\} \times I) \rightarrow T$ from $(f \cdot g) \cdot h$ to $f \cdot(g \cdot h)$ is given as follows:

$$
H_{1}\left(t_{1}, \ldots, t_{n-1}, t_{n}, u\right)= \begin{cases}f\left(t_{1}, \ldots, t_{n-1}, \frac{4 t_{n}}{u+1}\right), & 0<t_{n} \leq \frac{u+1}{4} \\ g\left(t_{1}, \ldots, t_{n-1}, 4 t_{n}-u-1\right), & \frac{u+1}{4} \leq t_{n} \leq \frac{u+2}{4} \\ h\left(t_{1}, \ldots, t_{n-1}, \frac{4 t_{n}-2-u}{2-u}\right), & \frac{u+2}{4} \leq t_{n}<1\end{cases}
$$

Since the image of $H_{1}$ is contained in $s k_{n}(T)$, we have $\left(H_{1}\right)_{*}\left(\left[S^{n} \times I\right]\right)=0$ in $C_{n+1}(T)$. Hence $H_{1}$ becomes a cellular homotopy from $((f, \omega) \cdot(g, \tau)) \cdot(h, \eta)$ to $(f, \omega) \cdot((g, \tau) \cdot(h, \eta))$ for any $\omega, \tau, \eta \in \widetilde{W}_{n+1}$.

Finally we show the existence of unit and inverse with respect to the multiplication $\cdot$ Let $0: S^{n} \rightarrow T$ be the map given by $0\left(S^{n}\right)=\{*\}$. For a pointed cellular map $f: S^{n} \rightarrow T$, a homotopy $H_{2}$ from $f \cdot 0$ to $f$ is given as follows:

$$
H_{2}\left(t_{1}, \ldots, t_{n-1}, t_{n}, u\right)= \begin{cases}f\left(t_{1}, \ldots, t_{n-1}, \frac{2 t_{n}}{u+1}\right), & 0<t_{n} \leq \frac{u+1}{2} \\ *, & \frac{u+1}{2} \leq t_{n}<1\end{cases}
$$


A homotopy $H_{3}$ from $0 \cdot f$ to $f$ can be given in a similar form. Moreover, a homotopy $H_{4}$ from $f \cdot f^{-1}$ to 0 is given as follows:

$$
H_{4}\left(t_{1}, \ldots, t_{n-1}, t_{n}, u\right)= \begin{cases}f\left(t_{1}, \ldots, t_{n-1}, \frac{2 t_{n}}{1-u}\right), & 0<t_{n} \leq \frac{1-u}{2} \\ *, & \frac{1-u}{2} \leq t_{n} \leq \frac{1+u}{2} \\ f\left(t_{1}, \ldots, t_{n-1}, \frac{-2 t_{n}+2}{1-u}\right), & \frac{u+1}{2} \leq t_{n}<1 .\end{cases}
$$

A homotopy $H_{5}$ from $f^{-1} \cdot f$ to 0 can be given in a similar form. These homotopies are all cellular and their images are contained in $s k_{n}(T)$. Hence $(f, \omega) \cdot(0,0)$ and $(0,0) \cdot(f, \omega)$ are homotopy equivalent to $(f, \omega)$, and $(f, \omega)$. $\left(f^{-1},-\omega\right)$ and $\left(f^{-1},-\omega\right) \cdot(f, \omega)$ are homotopy equivalent to $(0,0)$.

Theorem 3.1. For $n \geq 1$, the multiplication $\cdot$ gives the structure of a group on $\widehat{\pi}_{n}(T, \rho)$ and when $n \geq 2$, it becomes commutative.

Proof. The former part has already been proved. When $n \geq 2$, for two pointed cellular maps $f, g: S^{n} \rightarrow T, f \cdot g$ is homotopy equivalent to $g \cdot f$. A homotopy between them is described in every textbook of homotopy theory, and it is easy to see that the image of this homotopy is also contained in $s k_{n}(T)$. Hence $(f \cdot g, 0)$ is homotopy equivalent to $(g \cdot f, 0)$.

Definition 3.2. The group $\widehat{\pi}_{n}(T, \rho)$ is called the $n$-th homotopy group of $T$ modified by the homomorphism $\rho$.

Let $\zeta: \widehat{\pi}_{n}(T, \rho) \rightarrow \pi_{n}(T)$ denote the surjection obtained by forgetting elements of $\widetilde{W}_{n+1}$. Then we have the following:

Theorem 3.3. $\quad$ There is an exact sequence

$$
\pi_{n+1}(T) \stackrel{\widetilde{\rho}}{\rightarrow} \widetilde{W}_{n+1} \stackrel{a}{\rightarrow} \widehat{\pi}_{n}(T, \rho) \stackrel{\zeta}{\rightarrow} \pi_{n}(T) \rightarrow 0,
$$

where the map $\widetilde{\rho}$ is given by

$$
\widetilde{\rho}: \pi_{n+1}(T) \stackrel{\text { Hurewicz }}{\longrightarrow} H_{n+1}(T) \stackrel{H_{n+1}(\rho)}{\longrightarrow} H_{n+1}\left(W_{*}\right) \subset \widetilde{W}_{n+1}
$$

and the map a by $a(\omega)=[(0, \omega)] \in \widehat{\pi}_{n}(T, \rho)$.

Proof. The cellular approximation theorem implies that $\operatorname{Im} a=\operatorname{Ker} \zeta$. Hence we have only to show that $\operatorname{Ker} a=\operatorname{Im} \widetilde{\rho}$. For $\omega \in \widetilde{W}_{n+1}$, the pair $(0, \omega)$ is homotopy equivalent to $(0,0)$ if and only if there is a cellular homotopy $H:\left(S^{n} \times I\right) /(\{*\} \times I) \rightarrow T$ from 0 to 0 such that $(-1)^{n+1} \rho H_{*}\left(\left[S^{n} \times I\right]\right)=\omega$. 
Since $H\left(S^{n} \times \partial I\right)=\{*\}, H$ gives a pointed cellular map $H^{\prime}: S^{n+1} \rightarrow T$. Then $\omega$ is equal to the image of $(-1)^{n+1}\left[H^{\prime}\right] \in \pi_{n+1}(T)$ by $\widetilde{\rho}$, therefore Ker $a \subset \operatorname{Im} \widetilde{\rho}$. The opposite inclusion $\operatorname{Im} \widetilde{\rho} \subset$ Ker $a$ can be verified by regarding a pointed cellular map $S^{n+1} \rightarrow T$ as a cellular homotopy from 0 to 0 .

\section{§3.2. A homomorphism from a modified homotopy group}

For a pair $(f, \omega)$ as in the previous subsection, let $\rho(f, \omega)=\rho f_{*}\left(\left[S^{n}\right]\right)+$ $\partial \omega \in W_{n}$.

Proposition 3.4. The above $\rho(f, \omega)$ gives rise to a homomorphism

$$
\rho: \widehat{\pi}_{n}(T, \rho) \rightarrow W_{n}
$$

and $\operatorname{Im} \rho$ is contained in $\operatorname{Ker}\left(\partial: W_{n} \rightarrow W_{n-1}\right)$.

Proof. If $H:\left(S^{n} \times I\right) /(\{*\} \times I) \rightarrow T$ is a cellular homotopy from $(f, \omega)$ to $\left(f^{\prime}, \omega^{\prime}\right)$, then

$$
\partial H_{*}\left(\left[S^{n} \times I\right]\right)=(-1)^{n}\left(f_{*}^{\prime}\left(\left[S^{n}\right]\right)-f_{*}\left(\left[S^{n}\right]\right)\right)
$$

in $C_{n}(T)$ and $\rho H_{*}\left(\left[S^{n} \times I\right]\right)=(-1)^{n+1}\left(\omega^{\prime}-\omega\right)$. Hence we have

$$
\begin{aligned}
\rho(f, \omega) & =\rho f_{*}\left(\left[S^{n}\right]\right)+\partial \omega \\
& \left.=\rho f_{*}^{\prime}\left(\left[S^{n}\right]\right)+(-1)^{n+1} \partial \rho H_{*}\left(\left[S^{n} \times I\right]\right)\right)+\partial \omega \\
& =\rho f_{*}^{\prime}\left(\left[S^{n}\right]\right)+\partial\left(\omega^{\prime}-\omega\right)+\partial \omega \\
& =\rho\left(f^{\prime}, \omega^{\prime}\right),
\end{aligned}
$$

therefore $\rho(f, \omega)$ gives rise to a homomorphism from $\widehat{\pi}_{n}(T, \rho)$. The inclusion $\operatorname{Im} \rho \subset \operatorname{Ker}\left(\partial: W_{n} \rightarrow W_{n-1}\right)$ is obvious.

The exact sequence in Theorem 3.3 implies the following corollaries:

Corollary 3.5. There is an exact sequence

$$
\pi_{n+1}(T) \stackrel{\widetilde{\rho}}{\rightarrow} H_{n+1}\left(W_{*}\right) \stackrel{a}{\rightarrow} \widehat{\pi}_{n}(T, \rho) \stackrel{\zeta \oplus \rho}{\longrightarrow} \pi_{n}(T) \oplus \operatorname{Ker} \partial \stackrel{c l}{\longrightarrow} H_{n}\left(W_{*}\right) \rightarrow 0,
$$

where $\operatorname{Ker} \partial=\operatorname{Ker}\left(\partial: W_{n} \rightarrow W_{n-1}\right)$ and $\operatorname{cl}(x, \omega)=\widetilde{\rho}(x)-[\omega]$.

Corollary 3.6. For $n \geq 1$, let

$$
\widehat{\pi}_{n}(T, \rho)_{0}=\operatorname{Ker}\left(\rho: \widehat{\pi}_{n}(T, \rho) \rightarrow W_{n}\right) .
$$

Then there is a long exact sequence

$$
\cdots \stackrel{\zeta}{\rightarrow} \pi_{n+1}(T) \stackrel{\widetilde{\rho}}{\rightarrow} H_{n+1}\left(W_{*}\right) \stackrel{a}{\rightarrow} \widehat{\pi}_{n}(T, \rho)_{0} \stackrel{\zeta}{\rightarrow} \pi_{n}(T) \stackrel{\widetilde{\rho}}{\rightarrow} \cdots .
$$




\section{§3.3. Comparison to the homotopy group of the homotopy fiber of $\rho$}

In this subsection we show that $\widehat{\pi}_{n}(T, \rho)_{0}$ is canonically isomorphic to the $n$-th homotopy group of the homotopy fiber of the map $\rho$. Here we work with the category of simplicial sets, not with the category of CW-complexes. Let us first recall Dold-Kan correspondence. See [11] for a concrete account.

Let $A$ be a simplicial abelian group. Then we obtain the chain complex associated to $A$ by $A_{*}=\left(A_{n}, \partial=\sum_{i}(-1)^{i} \partial_{i}\right)$. We can define another chain complex $N A_{*}$ called the normalized chain complex of $A$. It is a subcomplex of $A_{*}$ such that the inclusion is a quasi-isomorphism.

For a chain complex $W_{*}$ of abelian groups, we can construct a simplicial abelian group $\Gamma\left(W_{*}\right)$. The group of $n$-th simplexes of $\Gamma\left(W_{*}\right)$ is the direct sum of $W_{n}$ with the subgroup generated by degenerate simplexes, and the canonical projection

$$
\varphi: \Gamma\left(W_{*}\right)_{*} \rightarrow W_{*}
$$

is a homomorphism of chain complexes. The homotopy group of $\Gamma\left(W_{*}\right)$ is canonically isomorphic to the homology group of $W_{*}$ :

$$
\pi_{n}\left(\Gamma\left(W_{*}\right)\right) \simeq H_{n}\left(W_{*}\right) .
$$

Dold-Kan correspondence [11, Cor. III. 2.3] says that the functors $N$ and $\Gamma$ are mutually inverse.

Suppose $T=|K|$, the topological realization of a pointed simplicial set $K$. Let $\mathbb{Z} K$ denote the simplicial abelian group spanned by $K$ and $\mathbb{Z} K_{*}$ denote the chain complex associated to $\mathbb{Z} K$. We can regard $\rho$ as the homomorphism from $\mathbb{Z} K_{*}:$

$$
\rho: \mathbb{Z} K_{*} \rightarrow C_{*}(|K|) \rightarrow W_{*} .
$$

Then we have the map of simplicial sets

$$
\rho^{\sharp}: K \rightarrow \mathbb{Z} K=\Gamma\left(N \mathbb{Z} K_{*}\right) \hookrightarrow \Gamma\left(\mathbb{Z} K_{*}\right) \stackrel{\Gamma(\rho)}{\longrightarrow} \Gamma\left(W_{*}\right) .
$$

Lemma 3.7. The map

$$
\mathbb{Z} K_{*} \stackrel{\rho_{*}^{\sharp}}{\longrightarrow} \Gamma\left(W_{*}\right)_{*} \stackrel{\varphi}{\longrightarrow} W_{*}
$$

coincides with $\rho$.

Proof. We have $\mathbb{Z} K_{*}=N \mathbb{Z} K_{*} \oplus D_{*}$ and $\Gamma\left(W_{*}\right)_{*}=W_{*} \oplus D_{*}^{\prime}$ where $D_{*}$ and $D_{*}^{\prime}$ are subcomplexes generated by degenerate elements. Since $\rho_{*}^{\sharp}$ comes 
from the map of simplicial sets, it is described as

$$
\mathbb{Z} K_{*}=N \mathbb{Z} K_{*} \oplus D_{*} \stackrel{\rho \oplus \psi}{\longrightarrow} W_{*} \oplus D_{*}^{\prime}=\Gamma\left(W_{*}\right)_{*},
$$

where $\psi=\left.\rho_{*}^{\sharp}\right|_{D_{*}}$.

For $x \in \mathbb{Z} K_{*}$, take the decomposition $x=x_{N}+x_{D}$, where $x_{N} \in N \mathbb{Z} K_{*}$ and $x_{D} \in D_{*}$. Then $\varphi \rho_{*}^{\sharp}(x)=\rho\left(x_{N}\right)=\rho(x)-\rho\left(x_{D}\right)$. Since $\rho$ factors through $C_{*}(|K|)$, we have $\rho\left(x_{D}\right)=0$, hence $\varphi \rho_{*}^{\sharp}(x)=\rho(x)$.

Theorem 3.8. Let $\mathrm{Fib}_{\rho^{\sharp}}$ denote the homotopy fiber of $\rho^{\sharp}$. Then for $n \geq 1$ there exists a canonical isomorphism

$$
\pi_{n}\left(\mathrm{Fib}_{\rho^{\sharp}}\right) \simeq \widehat{\pi}_{n}(|K|, \rho)_{0}
$$

making the following diagram commutative up to multiplication by \pm 1 :

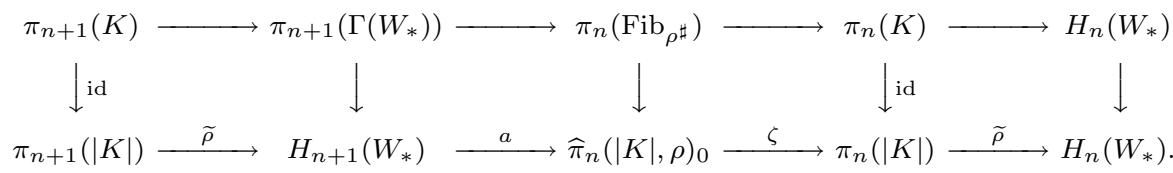

Proof. Take a Kan complex $K^{\prime}$ with an anodyne extension $K \rightarrow K^{\prime}$. Since $\Gamma\left(W_{*}\right)$ is also a Kan complex by [11, Lem. I. 3.4], there is an extension $\rho^{\prime \sharp}: K^{\prime} \rightarrow \Gamma\left(W_{*}\right)$ of $\rho^{\sharp}$. Let $\rho^{\prime}$ be the homomorphism of chain complexes given as follows:

$$
\mathbb{Z} K_{*}^{\prime} \stackrel{\rho^{\prime \sharp}}{\longrightarrow} \Gamma\left(W_{*}\right)_{*} \stackrel{\varphi}{\longrightarrow} W_{*} .
$$

It is easily shown that the image of any degenerate simplex of $K^{\prime}$ by $\rho^{\prime}$ is zero. Hence $\rho^{\prime}$ gives the homomorphism $C_{*}\left(\left|K^{\prime}\right|\right) \rightarrow W_{*}$. By Lemma 3.7 we have the commutative diagram

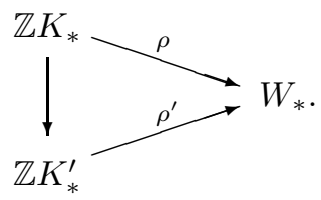

Therefore we may assume that $K$ itself is a Kan complex.

Let $\Delta[1]$ be the simplicial set represented by [1] and fix $\{0\} \in \Delta[1]_{0}=$ $\operatorname{Hom}([0],[1])$ as the base point. Let $\mathcal{H}_{\bullet}\left(\Delta[1], \Gamma\left(W_{*}\right)\right)$ denote the function complex from $\Delta[1]$ to $\Gamma\left(W_{*}\right)$ preserving the base point. Define $F$ to be the 
cartesian product of the following diagram:

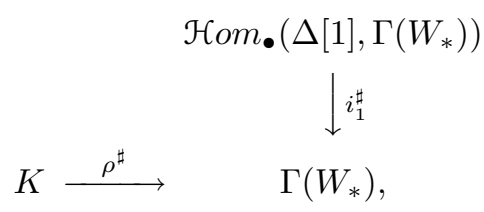

where $i_{1}^{\sharp}$ is the map taking composite with the injection $i_{1}:\{1\} \rightarrow \Delta[1]$. Then the topological realization of $F$ is homotopy equivalent to $\mathrm{Fib}_{\rho^{\sharp}}$.

Let $\Delta[n]$ be the simplicial set represented by $[n]$ and $\partial \Delta[n]$ its boundary. Note that the topological realization of $\Delta[n] / \partial \Delta[n]$ is the $n$-dimensional sphere $S^{n}$ with the usual cellular decomposition into two cells. Since $F$ is a Kan complex, the homotopy group of $F$ is given by the set of all maps from $\Delta[n] / \partial \Delta[n]$ to $F$ modulo simplicial homotopy.

Take a map of pointed simplicial sets $f: \Delta[n] / \partial \Delta[n] \rightarrow F$. Then we have two maps:

$$
\begin{aligned}
& f_{1}: \Delta[n] / \partial \Delta[n] \stackrel{f}{\longrightarrow} F \stackrel{\pi_{1}}{\longrightarrow} K, \\
& f_{2}: \Delta[n] / \partial \Delta[n] \stackrel{f}{\longrightarrow} F \stackrel{\pi_{2}}{\longrightarrow} \mathcal{H o m} \bullet\left(\Delta[1], \Gamma\left(W_{*}\right)\right),
\end{aligned}
$$

where $\pi_{1}$ and $\pi_{2}$ are the projections. Let

$$
\left|f_{1}\right|: S^{n}=|\Delta[n] / \partial \Delta[n]| \rightarrow|K|
$$

be the topological realization of $f_{1}$ and

$$
f_{2}^{\sharp}:(\Delta[n] \times \Delta[1]) /(\partial \Delta[n] \times \Delta[1]) \cup(\Delta[n] \times\{0\}) \rightarrow \Gamma\left(W_{*}\right)
$$

be the map corresponding to $f_{2}$. Let $[\Delta[n] \times \Delta[1]]$ be the fundamental chain and $\omega_{f_{2}}=\varphi f_{2 *}^{\sharp}([\Delta[n] \times \Delta[1]]) \in W_{n+1}$. Then

$$
\begin{aligned}
\partial \omega_{f_{2}} & =\varphi f_{2 *}^{\sharp}(\partial[\Delta[n] \times \Delta[1]]) \\
& =(-1)^{n} \varphi f_{2 *}^{\sharp}([\Delta[n] \times\{1\}]) \\
& =(-1)^{n} \varphi \rho^{\sharp} f_{1 *}([\Delta[n]]) \\
& =(-1)^{n} \rho f_{1 *}([\Delta[n]]) .
\end{aligned}
$$

Hence the pair $\left(\left|f_{1}\right|,(-1)^{n+1} \omega_{f_{2}}\right)$ gives an element of $\widehat{\pi}_{n}(|K|, \rho)_{0}$.

Let $f, g: \Delta[n] / \partial \Delta[n] \rightarrow F$ be maps of pointed simplicial sets and

$$
H:(\Delta[n] \times \Delta[1]) /(\partial \Delta[n] \times \Delta[1]) \rightarrow F
$$


a homotopy from $f$ to $g$. Let

$$
\begin{aligned}
& H_{1}:(\Delta[n] \times \Delta[1]) /(\partial \Delta[n] \times \Delta[1]) \stackrel{H}{\longrightarrow} F \stackrel{\pi_{1}}{\longrightarrow} K, \\
& H_{2}:(\Delta[n] \times \Delta[1]) /(\partial \Delta[n] \times \Delta[1]) \stackrel{H}{\longrightarrow} F \stackrel{\pi_{2}}{\longrightarrow} \mathcal{H o m} \bullet\left(\Delta[1], \Gamma\left(W_{*}\right)\right) .
\end{aligned}
$$

The map $H_{1}$ is a homotopy from $f_{1}$ to $g_{1}$. Let

$H_{2}^{\sharp}:(\Delta[n] \times \Delta[1] \times \Delta[1]) /(\partial \Delta[n] \times \Delta[1] \times \Delta[1]) \cup(\Delta[n] \times \Delta[1] \times\{0\}) \rightarrow \Gamma\left(W_{*}\right)$

the map corresponding to $H_{2}$. If we denote $\omega_{H_{2}}=\varphi H_{2 *}^{\sharp}([\Delta[n] \times \Delta[1] \times \Delta[1]]) \in$ $W_{n+2}$, then

$$
\begin{aligned}
\partial \omega_{H_{2}}= & (-1)^{n} \varphi H_{2 *}^{\sharp}([\Delta[n] \times\{1\} \times \Delta[1]]-[\Delta[n] \times\{0\} \times \Delta[1]] \\
& \quad-[\Delta[n] \times \Delta[1] \times\{1\}]) \\
= & (-1)^{n}\left(\omega_{g_{2}}-\omega_{f_{2}}\right)+(-1)^{n+1} \rho H_{1 *}([\Delta[n] \times \Delta[1]]) .
\end{aligned}
$$

Hence

$$
\left[\left(\left|f_{1}\right|,(-1)^{n+1} \omega_{f_{2}}\right)\right]=\left[\left(\left|g_{1}\right|,(-1)^{n+1} \omega_{g_{2}}\right)\right],
$$

which tells that $f \mapsto\left[\left(\left|f_{1}\right|,(-1)^{n+1} \omega_{f_{2}}\right)\right]$ gives rise to a map

$$
\pi_{n}(F) \rightarrow \widehat{\pi}_{n}(|K|, \rho)_{0} .
$$

Next we show that the above map is a homomorphism of groups. Let $f, g, h: \Delta[n] / \partial \Delta[n] \rightarrow F$ be maps of pointed simplicial sets and $\sigma: \Delta[n+1] \rightarrow$ $F$ a map such that $\partial_{n-1} \sigma=f, \partial_{n+1} \sigma=g, \partial_{n} \sigma=h$ and $\partial_{j} \sigma$ is the map collapsing to the base point for $j \leq n-2$. Then $[f]+[g]=[h]$ in $\pi_{n}(F)$ and any sum in $\pi_{n}(F)$ is described in this way. Let

$$
\begin{aligned}
\sigma_{1} & : \Delta[n+1] \stackrel{\sigma}{\longrightarrow} F \stackrel{\pi_{1}}{\longrightarrow} K, \\
\sigma_{2} & : \Delta[n+1] \stackrel{\sigma}{\longrightarrow} F \stackrel{\pi_{2}}{\longrightarrow} \mathcal{H o m} \bullet\left(\Delta[1], \Gamma\left(W_{*}\right)\right)
\end{aligned}
$$

and

$$
\sigma_{2}^{\sharp}:(\Delta[n+1] \times \Delta[1]) /(\Delta[n+1] \times\{0\}) \rightarrow \Gamma\left(W_{*}\right)
$$

the map corresponding to $\sigma_{2}$. If we denote $\omega_{\sigma_{2}}=\varphi \sigma_{2 *}^{\sharp}([\Delta[n+1] \times \Delta[1]]) \in$ $W_{n+2}$, then

$$
\begin{aligned}
\partial \omega_{\sigma_{2}} & =\varphi \sigma_{2 *}^{\sharp}\left([\partial \Delta[n+1] \times \Delta[1]]+(-1)^{n+1}[\Delta[n+1] \times\{1\}]\right) \\
& =(-1)^{n-1}\left(\omega_{f_{2}}+\omega_{g_{2}}-\omega_{h_{2}}\right)+(-1)^{n+1} \rho \sigma_{1 *}([\Delta[n+1]]) .
\end{aligned}
$$

Hence $\sigma_{1}$ is a homotopy from $f_{1} \cdot g_{1}$ to $h_{1}$ such that

$$
\rho \sigma_{1 *}([\Delta[n+1]])=\omega_{h_{2}}-\omega_{f_{2}}-\omega_{g_{2}}+(-1)^{n+1} \partial \omega_{\sigma_{2}} .
$$


Hence

$$
\left[\left(\left|h_{1}\right|,(-1)^{n+1} \omega_{h_{2}}\right)\right]=\left[\left(\left|f_{1}\right|,(-1)^{n+1} \omega_{f_{2}}\right)\right]+\left[\left(\left|g_{1}\right|,(-1)^{n+1} \omega_{g_{2}}\right)\right],
$$

which tells that the map $\pi_{n}(F) \rightarrow \widehat{\pi}_{n}(|K|, \rho)_{0}$ is a homomorphism of groups.

It is obvious that the diagram

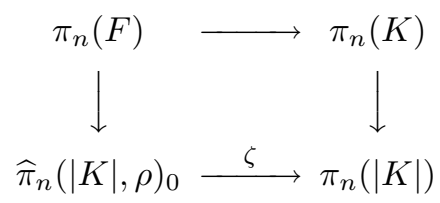

is commutative. Consider the following diagram:

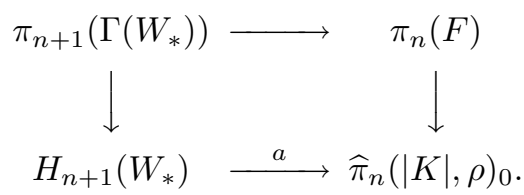

In the above, the upper horizontal arrow is obtained from the map of simplicial sets

$$
\mathcal{H}_{\bullet} \bullet\left(\Delta[1] / \partial \Delta[1], \Gamma\left(W_{*}\right)\right) \longrightarrow F=K \underset{\Gamma\left(W_{*}\right)}{\times} \mathcal{H o m} \bullet\left(\Delta[1], \Gamma\left(W_{*}\right)\right)
$$

given by $\gamma \mapsto(*, \widetilde{\gamma})$, where $*$ is the base point of $K$ and $\widetilde{\gamma}$ is the element of $\mathcal{H} \operatorname{Hom}_{\bullet}\left(\Delta[1], \Gamma\left(W_{*}\right)\right)$ given by $\gamma$. Take a map of pointed simplicial sets

$$
\omega: \Delta[n] / \partial \Delta[n] \rightarrow \mathcal{H}_{\bullet}\left(\Delta[1] / \partial \Delta[1], \Gamma\left(W_{*}\right)\right)
$$

and let

$$
\omega^{\sharp}: \Delta[n] \times \Delta[1] / \partial(\Delta[n] \times \Delta[1]) \rightarrow \Gamma\left(W_{*}\right)
$$

be the map corresponding to $\omega$. Then the image of

$$
\left[\omega^{\sharp}\right] \in \pi_{n+1}\left(\Gamma\left(W_{*}\right)\right) \simeq \pi_{n}\left(\mathcal{H o m} \bullet\left(\Delta[1] / \partial \Delta[1], \Gamma\left(W_{*}\right)\right)\right)
$$

by the map

$$
\pi_{n+1}\left(\Gamma\left(W_{*}\right)\right) \rightarrow \pi_{n}(F) \rightarrow \widehat{\pi}_{n}(|K|, \rho)_{0}
$$

is $\left[\left(0,(-1)^{n+1} \varphi \omega_{*}^{\sharp}([\Delta[n] \times \Delta[1]])\right)\right]$. This shows that the above diagram is commutative up to multiplication by $(-1)^{n+1}$.

It is obvious from the five lemma that $\pi_{n}(F) \rightarrow \widehat{\pi}_{n}(|K|, \rho)_{0}$ is an isomorphism. 


\section{§3.4. A functoriality of modified homotopy groups}

Let $T$ and $T^{\prime}$ be pointed CW-complexes and let $W_{*}$ and $W_{*}^{\prime}$ be chain complexes. Let $\alpha: T \rightarrow T^{\prime}$ be a pointed cellular map and let $\rho: C_{*}(T) \rightarrow W_{*}$, $\rho^{\prime}: C_{*}\left(T^{\prime}\right) \rightarrow W_{*}^{\prime}$ and $\beta: W_{*} \rightarrow W_{*}^{\prime}$ be homomorphisms of chain complexes that make the diagram

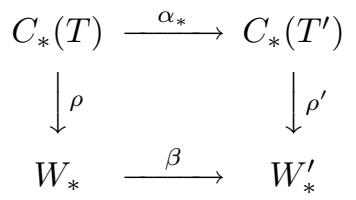

commutative up to a homotopy $\Phi$. In other words, there is a homomorphism $\Phi: C_{*}(T) \rightarrow W_{*+1}^{\prime}$ satisfying $\rho^{\prime} \alpha_{*}-\beta \rho=\partial \Phi+\Phi \partial$.

Proposition 3.9. $\quad$ Under the above notations, we can define a homomorphism

$$
(\alpha, \beta, \Phi)_{*}: \widehat{\pi}_{n}(T, \rho) \rightarrow \widehat{\pi}_{n}\left(T^{\prime}, \rho^{\prime}\right)
$$

by $[(f, \omega)] \mapsto\left[\left(\alpha f, \beta(\omega)-\Phi f_{*}\left(\left[S^{n}\right]\right)\right)\right]$. This homomorphism enjoys the following functorial property: Let $\alpha: T \rightarrow T^{\prime}$ and $\alpha^{\prime}: T^{\prime} \rightarrow T^{\prime \prime}$ be pointed cellular maps and let $\beta: W_{*} \rightarrow W_{*}^{\prime}$ and $\beta^{\prime}: W_{*}^{\prime} \rightarrow W_{*}^{\prime \prime}$ be homomorphisms of chain complexes. We assume that the squares

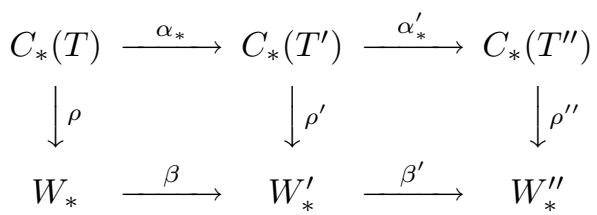

are commutative up to homotopies $\Phi$ and $\Phi^{\prime}$ respectively. Then

$$
\left(\alpha^{\prime}, \beta^{\prime}, \Phi^{\prime}\right)_{*}(\alpha, \beta, \Phi)_{*}=\left(\alpha^{\prime} \alpha, \beta^{\prime} \beta, \beta^{\prime} \Phi+\Phi^{\prime} \alpha_{*}\right)_{*}: \widehat{\pi}_{n}(T, \rho) \rightarrow \widehat{\pi}_{n}\left(T^{\prime \prime}, \rho^{\prime \prime}\right) .
$$

Proof. Let $f, f^{\prime}: S^{n} \rightarrow T$ be pointed cellular maps and $\omega, \omega^{\prime} \in \widetilde{W}_{n+1}$. If $H:\left(S^{n} \times I\right) /(\{*\} \times I) \rightarrow T$ is a cellular homotopy from $(f, \omega)$ to $\left(f^{\prime}, \omega^{\prime}\right)$, then

$$
\begin{aligned}
(-1)^{n+1} \rho^{\prime} \alpha_{*} & H_{*}\left(\left[S^{n} \times I\right]\right) \\
= & (-1)^{n+1} \beta \rho H_{*}\left(\left[S^{n} \times I\right]\right)+(-1)^{n+1} \partial \Phi H_{*}\left(\left[S^{n} \times I\right]\right) \\
& +(-1)^{n+1} \Phi \partial H_{*}\left(\left[S^{n} \times I\right]\right) \\
\equiv & \left(\beta\left(\omega^{\prime}\right)-\Phi f_{*}^{\prime}\left(\left[S^{n}\right]\right)\right)-\left(\beta(\omega)-\Phi f_{*}\left(\left[S^{n}\right]\right)\right)
\end{aligned}
$$

modulo Im $\partial$. This tells that the map $\alpha H:\left(S^{n} \times I\right) /(\{*\} \times I) \rightarrow T^{\prime}$ is a cellular homotopy from $\left(\alpha f, \beta(\omega)-\Phi f_{*}\left(\left[S^{n}\right]\right)\right)$ to $\left(\alpha f^{\prime}, \beta\left(\omega^{\prime}\right)-\Phi f_{*}^{\prime}\left(\left[S^{n}\right]\right)\right)$. 
Hence $(\alpha, \beta, \Phi)_{*}$ is well-defined. The functorial property can be shown by an easy calculation.

Proposition 3.10. Under the above notations, we have a commutative diagram

$$
\begin{array}{ccc}
\widehat{\pi}_{n}(T, \rho) \stackrel{\rho}{\longrightarrow} & W_{n} \\
\downarrow(\alpha, \beta, \Phi)_{*} & & \downarrow \beta \\
\widehat{\pi}_{n}\left(T^{\prime}, \rho^{\prime}\right) \stackrel{\rho^{\prime}}{\longrightarrow} & W_{n}^{\prime} .
\end{array}
$$

Proof. For a pointed cellular map $f: S^{n} \rightarrow T$,

$$
\rho^{\prime} \alpha_{*} f_{*}\left(\left[S^{n}\right]\right)-\beta \rho f_{*}\left(\left[S^{n}\right]\right)=\partial \Phi f_{*}\left(\left[S^{n}\right]\right) .
$$

Hence

$$
\begin{aligned}
\rho^{\prime}(\alpha, \beta, \Phi)_{*}([(f, \omega)]) & =\rho^{\prime}\left(\left[\left(\alpha f, \beta(\omega)-\Phi f_{*}\left(\left[S^{n}\right]\right)\right)\right]\right) \\
& =\rho^{\prime} \alpha_{*} f_{*}\left(\left[S^{n}\right]\right)+\partial\left(\beta(\omega)-\Phi f_{*}\left(\left[S^{n}\right]\right)\right) \\
& =\beta\left(\rho f_{*}\left(\left[S^{n}\right]\right)+\partial \omega\right) \\
& =\beta \rho([(f, \omega)]) .
\end{aligned}
$$

\section{$\S 4$. Definition of Arithmetic $K$-groups}

\section{§4.1. Triviality of the Bott-Chern form of a degenerate element}

In this subsection we prove that the Bott-Chern form of a degenerate element of $S_{*} \mathfrak{A}$ vanishes. We begin with the following lemma:

Lemma 4.1. For any $E \in S_{n} \mathfrak{A}$, we have

$$
\begin{aligned}
& \operatorname{Cub}\left(s_{0} E\right)=s_{1}^{-1} \operatorname{Cub}(E), \\
& \operatorname{Cub}\left(s_{n} E\right)=s_{n}^{1} \operatorname{Cub}(E)
\end{aligned}
$$

and if $1 \leq i \leq n-1$, then we have

$$
\operatorname{Cub}\left(s_{i} E\right)=\tau_{i} \operatorname{Cub}\left(s_{i} E\right),
$$

where $\tau_{i} \in \mathfrak{S}_{n}$ is the transposition of $i$ and $i+1$. 
Proof. In order to prove the lemma, we use [6, Prop. 4.5], in which all faces of $\operatorname{Cub}(E)$ for $E \in S_{n} \mathfrak{A}$ are described. Using this proposition, we can show that

$$
\begin{gathered}
\partial_{1}^{-1} \operatorname{Cub}\left(s_{0} E\right)=0, \\
\partial_{1}^{0} \operatorname{Cub}\left(s_{0} E\right)=\partial_{1}^{1} \operatorname{Cub}\left(s_{0} E\right)=\operatorname{Cub}(E) .
\end{gathered}
$$

Hence $\operatorname{Cub}\left(s_{0} E\right)=s_{1}^{-1} \operatorname{Cub}(E)$. The second identity can be shown in a similar way. The last identity follows from

$$
\partial_{i}^{j} \operatorname{Cub}\left(s_{i} E\right)=\partial_{i+1}^{j} \operatorname{Cub}\left(s_{i} E\right)
$$

for $1 \leq i \leq n-1$ and $-1 \leq j \leq 1$, which can be shown also by using [6, Prop. 4.5].

Let $\mathfrak{S}_{n}$ denote the $n$-th symmetric group. For $\sigma \in \mathfrak{S}_{n}$ and an exact $n$ cube $\mathcal{F}$ of a small exact category $\mathfrak{A}$, let $\sigma \mathcal{F}$ be an exact $n$-cube defined by $(\sigma \mathcal{F})_{\alpha_{1}, \ldots, \alpha_{n}}=\mathcal{F}_{\alpha_{\sigma(1)}, \alpha_{\sigma(2)}, \ldots, \alpha_{\sigma(n)}}$. Let $S_{n} \subset \mathbb{Z} C_{n} \mathfrak{A}$ be the subgroup generated by exact $n$-cubes $\mathcal{F}$ such that $\tau_{i} \mathcal{F}=\mathcal{F}$ for some integer $i$ with $1 \leq i \leq n-1$. Set

$$
\operatorname{Cub}_{n}(\mathfrak{A})=\mathbb{Z} C_{n} \mathfrak{A} /\left(D_{n}+S_{n}\right) .
$$

Lemma 4.2. We have $\partial S_{n} \subset S_{n-1}$. Hence $\operatorname{Cub}_{*}(\mathfrak{A})=\left(\operatorname{Cub}_{n}(\mathfrak{A}), \partial\right)$ becomes a chain complex.

Proof. Let $\mathcal{F}$ be an exact $n$-cube satisfying $\tau_{i} \mathcal{F}=\mathcal{F}$. If $k<i$, then $\partial_{k}^{j} \mathcal{F}=\partial_{k}^{j} \tau_{i} \mathcal{F}=\tau_{i-1} \partial_{k}^{j} \mathcal{F}$ and if $k>i+1$, then $\partial_{k}^{j} \mathcal{F}=\partial_{k}^{j} \tau_{i} \mathcal{F}=\tau_{i} \partial_{k}^{j} \mathcal{F}$. Furthermore, $\tau_{i} \mathcal{F}=\mathcal{F}$ implies that $\partial_{i}^{j} \mathcal{F}=\partial_{i+1}^{j} \mathcal{F}$. Hence

$$
\partial \mathcal{F}=\sum_{k \neq i, i+1} \sum_{j=-1}^{1}(-1)^{k+j+1} \partial_{k}^{j} \mathcal{F} \in S_{n-1} .
$$

Lemma 4.3. Let $\mathcal{F}$ be an exact hermitian $n$-cube on a complex algebraic manifold $M$. For any $\sigma \in \mathfrak{S}_{n}$, there is a canonical isometry $\sigma(\lambda \mathcal{F}) \simeq \lambda(\sigma \mathcal{F})$.

Proof. As seen in [6, §3], the emi- $n$-cube $\lambda \mathcal{F}$ is written as $\lambda_{n} \cdots \lambda_{2} \lambda_{1} \mathcal{F}$, where each $\lambda_{i}$ is an endomorphism of the chain complex $\widetilde{\mathbb{Z}} \widehat{C}_{*}(M)$, and it is easy to see that $\sigma\left(\lambda_{i} \mathcal{F}\right)=\lambda_{\sigma(i)}(\sigma \mathcal{F})$. Hence it is sufficient to show the existence of a canonical isometry $\lambda_{i} \lambda_{j} \simeq \lambda_{j} \lambda_{i}$. For simplicity, we prove it only in the case of $n=2$. 
For an exact hermitian 2-cube $\mathcal{F}=\left\{\overline{E_{i, j}}\right\}, \lambda_{2} \lambda_{1} \mathcal{F}$ is given as follows:

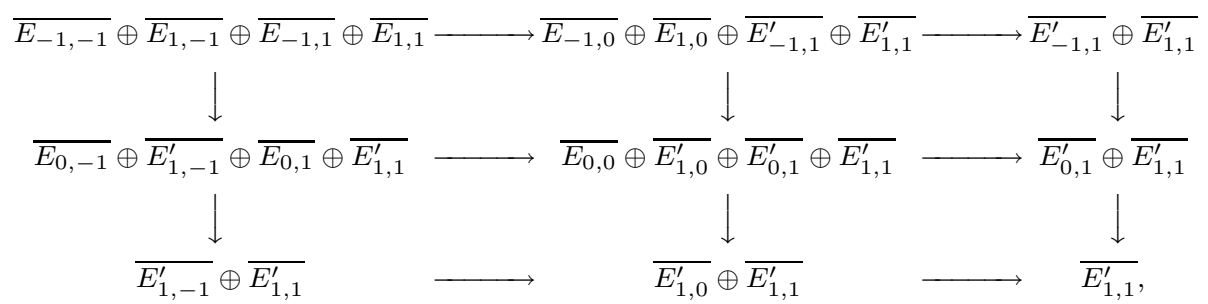

where $\overline{E_{i, j}^{\prime}}$ is the same vector bundle as $\overline{E_{i, j}}$ equipped with the metric induced from $\overline{E_{0,0}}$. On the other hand, $\lambda_{1} \lambda_{2} \mathcal{F}$ is given as follows:

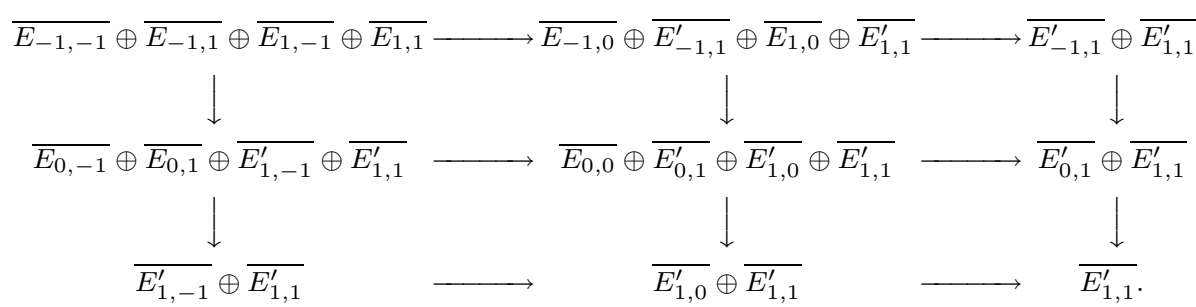

Hence an isometry $\lambda_{2} \lambda_{1} \mathcal{F} \simeq \lambda_{1} \lambda_{2} \mathcal{F}$ is given by appropriate permutations of direct summands.

Theorem 4.4. The Bott-Chern form of a degenerate element of $\widehat{S}_{n}(M)$ is zero.

Proof. For an integer $i$ with $1 \leq i \leq n-1$, let $t_{i}:\left(\mathbb{P}^{1}\right)^{n} \rightarrow\left(\mathbb{P}^{1}\right)^{n}$ denote the involution interchanging the $i$-th and the $(i+1)$-th components. Then by [13, Prop. 2.1] and Lemma 4.3, there is an isometry $t_{i}^{*} \operatorname{tr}_{n}(\lambda \mathcal{F}) \simeq \operatorname{tr}_{n}\left(\lambda \tau_{i} \mathcal{F}\right)$. Furthermore, it follows from the definition of $T_{n}$ that $t_{i}^{*} T_{n}=-T_{n}$. Hence if $\tau_{i} \mathcal{F}=\mathcal{F}$, then

$$
\begin{aligned}
\operatorname{ch}_{n}(\mathcal{F}) & =\int_{\left(\mathbb{P}^{1}\right)^{n}} \operatorname{ch}_{0}\left(\operatorname{tr}_{n}(\lambda \mathcal{F})\right) \wedge T_{n} \\
& =\int_{\left(\mathbb{P}^{1}\right)^{n}} t_{i}^{*}\left(\operatorname{ch}_{0}\left(\operatorname{tr}_{n}(\lambda \mathcal{F})\right) \wedge T_{n}\right)=-\operatorname{ch}_{n}(\mathcal{F}),
\end{aligned}
$$

therefore $\operatorname{ch}_{n}(\mathcal{F})=0$.

By Lemma 4.1, the cube $\operatorname{Cub}(E)$ associated with a degenerate element $E \in \widehat{S}_{n}(X)$ is either a degenerate cube or a cube satisfying $\tau_{i} \mathcal{F}=\mathcal{F}$ for some $1 \leq i \leq n-2$. Hence we can say that $\operatorname{ch}_{n-1}(E)=0$. 


\section{§4.2. Definition of higher arithmetic $K$-theory}

Let $X$ be a proper arithmetic variety. Let $X(\mathbb{C})$ denote the compact complex manifold consisting of $\mathbb{C}$-valued points on $X$ and $F_{\infty}: X(\mathbb{C}) \rightarrow X(\mathbb{C})$ the complex conjugation. The real Deligne cohomology of $X$ is the $\bar{F}_{\infty}^{*}$-invariant part of that of $X(\mathbb{C})$ :

$$
H_{\mathcal{D}}^{n}(X, \mathbb{R}(p))=H_{\mathcal{D}}^{n}(X(\mathbb{C}), \mathbb{R}(p))^{\bar{F}_{\infty}^{*}=\mathrm{id}} .
$$

Hence if we set

$$
\mathcal{D}^{n}(X, p)=\mathcal{D}^{n}(X(\mathbb{C}), p)^{\bar{F}_{\infty}^{*}=\mathrm{id}},
$$

then we have an isomorphism

$$
H^{n}\left(\mathcal{D}^{*}(X, p), d_{\mathcal{D}}\right) \simeq H_{\mathcal{D}}^{n}(X, \mathbb{R}(p))
$$

By a hermitian vector bundle on $X$ we mean a pair $\bar{E}=(E, h)$ of a vector bundle $E$ on $X$ and an $F_{\infty}$-invariant smooth hermitian metric $h$ on $E(\mathbb{C})$. An exact hermitian $n$-cube on $X$ is an exact $n$-cube made of hermitian vector bundles on $X$. Since the Chern form $\operatorname{ch}_{0}(\bar{E})$ is contained in $\oplus_{p} \mathcal{D}^{2 p}(X, p)$, the BottChern form of an exact hermitian $n$-cube on $X$ is contained in $\oplus_{p} \mathcal{D}^{2 p-n}(X, p)$.

Let $\widehat{\mathcal{P}}(X)$ be the category of hermitian vector bundles on $X, \widehat{S}(X)$ the $S$-construction of $\widehat{\mathcal{P}}(X)$, and $\widehat{\mathrm{Cub}}_{*}(X)=\operatorname{Cub}_{*}(\widehat{\mathcal{P}}(X))$. If we set $\mathcal{D}_{n}(X)=$ $\oplus_{p} \mathcal{D}^{2 p-n}(X, p)$, then by Theorem 4.4 we can obtain a homomorphism of chain complexes

$$
\operatorname{ch}: C_{*}(|\widehat{S}(X)|) \stackrel{\mathrm{Cub}}{\longrightarrow} \widehat{\mathrm{Cub}}_{*}(X)[1] \stackrel{\mathrm{ch}}{\longrightarrow} \mathcal{D}_{*}(X)[1] .
$$

Definition 4.5. The $n$-th arithmetic $K$-group $\widehat{K}_{n}(X)$ of $X$ is the $(n+$ 1)-th homotopy group of $|\widehat{S}(X)|$ modified by ch:

$$
\widehat{K}_{n}(X)=\widehat{\pi}_{n+1}(|\widehat{S}(X)|, \mathrm{ch}) .
$$

Applying Theorem 3.3 to the present context, we can obtain the following:

Theorem 4.6. $\quad$ There is an exact sequence

$$
K_{n+1}(X) \rightarrow \widetilde{\mathcal{D}}_{n+1}(X) \rightarrow \widehat{K}_{n}(X) \rightarrow K_{n}(X) \rightarrow 0,
$$

where $\widetilde{\mathcal{D}}_{n+1}(X)=\mathcal{D}_{n+1}(X) / \operatorname{Im} d_{\mathcal{D}}$.

As we mentioned in $\S 1$, the 0 -th arithmetic $K$-group has already been defined by Gillet and Soulé in [9]. Let us recall their definition again. 
Consider a pair $(\bar{E}, \omega)$ of a hermitian vector bundle $\bar{E}$ on $X$ and $\omega \epsilon$ $\widetilde{\mathcal{D}}_{1}(X)$. Let $\widehat{\mathcal{K}}_{0}(X)$ be the abelian group generated by all such pairs modulo the subgroup generated by

$$
\left(\overline{E^{\prime}}, \omega^{\prime}\right)+\left(\overline{E^{\prime \prime}}, \omega^{\prime \prime}\right)-\left(\bar{E}, \omega^{\prime}+\omega^{\prime \prime}-\operatorname{ch}_{1}(\varepsilon)\right)
$$

for all short exact sequences $\mathcal{E}: 0 \rightarrow \overline{E^{\prime}} \rightarrow \bar{E} \rightarrow \overline{E^{\prime \prime}} \rightarrow 0$ and $\omega^{\prime}, \omega^{\prime \prime} \in \widetilde{\mathcal{D}}_{1}(X)$. We denote by $[(\bar{E}, \omega)]$ the element of $\widehat{\mathcal{K}}_{0}(X)$ represented by a pair $(\bar{E}, \omega)$.

Strictly speaking, the group $\widehat{\mathcal{K}}_{0}(X)$ is different from the one defined by Gillet and Soulé up to a constant factor. This results from a difference of Chern-Weil forms. In fact, they think of the Chern-Weil form of $\bar{E}$ as the following real form:

$$
\operatorname{Tr}\left(\exp \left(\frac{-K_{\bar{E}}}{2 \pi \sqrt{-1}}\right)\right)
$$

Hence the $(p, p)$-part of the above form is equal to $\frac{1}{(2 \pi \sqrt{-1})^{p}} \operatorname{ch}_{0}(\bar{E})^{(p, p)}$. For $\omega=\sum_{p} \omega_{p} \in \oplus_{p} \mathcal{D}^{2 p-1}(X, p)=\mathcal{D}_{1}(X)$, let

$$
\Theta(\omega)=\sum_{p} \frac{2}{(2 \pi \sqrt{-1})^{p-1}} \omega_{p},
$$

then $\Theta(\omega)$ is a real form such that $-\Theta\left(\operatorname{ch}_{1}(\varepsilon)\right)$ modulo $\operatorname{Im} \partial+\operatorname{Im} \bar{\partial}$ is the BottChern secondary characteristic class of $\mathcal{E}$. Hence $(\bar{E}, \omega) \mapsto(\bar{E}, \Theta(\omega))$ gives an isomorphism from $\widehat{\mathcal{K}}_{0}(X)$ to Gillet and Soulé's arithmetic $K_{0}$-group of $X$.

Theorem 4.7. There is a canonical isomorphism

$$
\widehat{\alpha}: \widehat{\mathcal{K}}_{0}(X) \simeq \widehat{\pi}_{1}(|\widehat{S}(X)|, \operatorname{ch})=\widehat{K}_{0}(X) .
$$

Proof. Since $\widehat{S}_{1}(X)$ is the set of all hermitian vector bundles on $X$ and $\widehat{S}_{0}(X)=\{*\}$, any hermitian vector bundle $\bar{E}$ on $X$ gives a pointed simplicial loop $l_{\bar{E}}: S^{1} \rightarrow|\widehat{S}(X)|$. Moreover, any short exact sequence $\mathcal{E}: 0 \rightarrow \overline{E^{\prime}} \rightarrow \bar{E} \rightarrow$ $\overline{E^{\prime \prime}} \rightarrow 0$ gives a 2 -simplex $\Delta_{\varepsilon}$ in $\widehat{S}(X)$ whose faces are $\partial_{0} \Delta_{\varepsilon}=\overline{E^{\prime \prime}}, \partial_{1} \Delta_{\varepsilon}=\bar{E}$ and $\partial_{2} \Delta_{\varepsilon}=\overline{E^{\prime}}$. If we regard $\Delta_{\mathcal{E}}$ as a cellular homotopy from $l_{\overline{E^{\prime}}} \cdot l_{\overline{E^{\prime \prime}}}$ to $l_{\bar{E}}$, then $\operatorname{ch}_{1}\left(\left(\Delta_{\mathcal{E}}\right)_{*}\left[S^{1} \times I\right]\right)=\operatorname{ch}_{1}(\mathcal{E})$. Hence we have

$$
\left[\left(l_{\overline{E^{\prime}}} \cdot l_{\overline{E^{\prime \prime}}}, 0\right)\right]=\left[\left(l_{\bar{E}}, \operatorname{ch}_{1}(\mathcal{E})\right)\right]
$$

in $\widehat{\pi}_{1}(|\widehat{S}(X)|, \mathrm{ch})$. This tells that $(\bar{E}, \omega) \mapsto\left(l_{\bar{E}},-\omega\right)$ gives rise to a homomorphism of groups

$$
\widehat{\alpha}: \widehat{\mathcal{K}}_{0}(X) \rightarrow \widehat{\pi}_{1}(|\widehat{S}(X)|, \operatorname{ch})=\widehat{K}_{0}(X) .
$$


Consider the following commutative diagram:

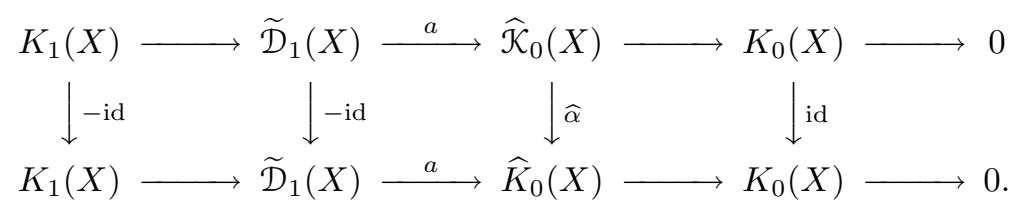

The upper sequence is exact by [9, Theorem 6.2$]$ and the lower one is exact by Theorem 4.6. Hence $\widehat{\alpha}$ is bijective by the five lemma.

Let $\varphi: X \rightarrow Y$ be a morphism of proper arithmetic varieties. Then we have a commutative diagram

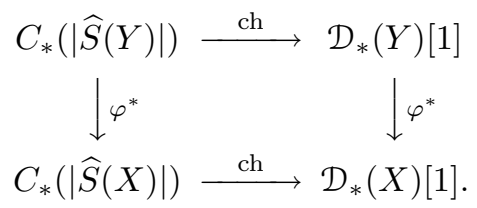

Hence we obtain a pull back homomorphism

$$
\widehat{\varphi}^{*}: \widehat{K}_{n}(Y) \rightarrow \widehat{K}_{n}(X)
$$

by $\widehat{\varphi}^{*}([(f, \omega)])=\left[\left(\varphi^{*} f, \varphi^{*} \omega\right)\right]$.

Let

$$
\operatorname{ch}_{n}: \widehat{K}_{n}(X) \rightarrow \mathcal{D}_{n}(X)
$$

be the map introduced in $\S 3.2$, that is,

$$
\operatorname{ch}_{n}([(f, \omega)])=\operatorname{ch}_{n}(f)-d_{\mathcal{D}} \omega
$$

where $\operatorname{ch}_{n}(f)=\operatorname{ch}\left(f_{*}\left(\left[S^{n+1}\right]\right)\right) \in \mathcal{D}_{n}(X)$. We call it the Chern form map. Applying Corollary 3.5, Corollary 3.6 and Theorem 3.8 to the present situation, we can obtain the following corollaries:

Corollary 4.8. There is an exact sequence

$$
\begin{aligned}
K_{n+1}(X) \stackrel{\rho}{\rightarrow} \oplus_{p} H_{\mathcal{D}}^{2 p-n-1}(X, \mathbb{R}(p)) \rightarrow \widehat{K}_{n}(X) \\
\quad \stackrel{\left(\zeta, \operatorname{ch}_{n}\right)}{\longrightarrow} K_{n}(X) \oplus \operatorname{Ker} d_{\mathcal{D}} \stackrel{c l}{\longrightarrow} \underset{p}{\oplus} H_{\mathcal{D}}^{2 p-n}(X, \mathbb{R}(p)) \rightarrow 0,
\end{aligned}
$$

where $\operatorname{cl}(x, \omega)=\rho(x)-[\omega]$ and $\rho$ is the Beilinson's regulator map. 
Corollary 4.9. Let $K M_{n}(X)$ denote the kernel of the Chern form map. Then there is a long exact sequence

$$
\cdots \rightarrow K_{n+1}(X) \stackrel{\rho}{\rightarrow} \underset{p}{\oplus} H_{\mathcal{D}}^{2 p-n-1}(X, \mathbb{R}(p)) \rightarrow K M_{n}(X) \rightarrow K_{n}(X) \stackrel{\rho}{\rightarrow} \cdots .
$$

Moreover, $K M_{n}(X)$ is canonically isomorphic to the $(n+1)$-th homotopy group of the homotopy fiber of

$$
\operatorname{ch}^{\sharp}: \widehat{S}(X) \rightarrow \Gamma\left(\mathcal{D}_{*}(X)[1]\right),
$$

where $\mathrm{ch}^{\sharp}$ is the map of pointed simplicial sets constructed from ch in the way as shown in $\S 3.3$.

We conclude this subsection by calculating the higher arithmetic $K$-theory of the ring of integers. Let $K$ be an algebraic number field and $\mathcal{O}_{K}$ its ring of integers. Let $X=\operatorname{Spec} \mathcal{O}_{K}$. Since $X(\mathbb{C})$ is zero-dimensional, $\mathcal{D}_{2 n}(X)=0$ if $n>0$ and $\mathcal{D}_{2 n+1}(X)$ is the recipient of the regulator map for $K_{2 n+1}\left(\mathcal{O}_{K}\right)$. Hence the exact sequence of Theorem 4.6 implies

$$
\widehat{K}_{2 n+1}\left(\mathcal{O}_{K}\right) \simeq K_{2 n+1}\left(\mathcal{O}_{K}\right)
$$

and

$$
\begin{aligned}
0 & \rightarrow \operatorname{Coker}\left(\rho: K_{2 n+1}\left(\mathcal{O}_{K}\right) \rightarrow(\underset{\sigma: K \hookrightarrow \mathbb{C}}{\oplus} \mathbb{R}(n))^{\bar{F}_{\infty}=\mathrm{id}}\right) \\
& \rightarrow \widehat{K}_{2 n}\left(\mathcal{O}_{K}\right) \rightarrow K_{2 n}\left(\mathcal{O}_{K}\right) \rightarrow 0
\end{aligned}
$$

where $K_{2 n}\left(\mathcal{O}_{K}\right)$ is a finite abelian group and the Borel's theorem [3] says that Coker $\rho$ is a quotient of a finite dimensional $\mathbb{R}$-vector space by a lattice.

\section{§4.3. Arakelov $K$-theory}

Let $M$ be a compact algebraic Kähler manifold with a Kähler metric $h_{M}$. Let $\mathcal{H}_{\mathbb{R}}^{n}(M)$ be the space of real harmonic forms on $M$ with respect to $h_{M}$ and $\mathcal{H}^{p, q}(M)$ the space of harmonic forms of type $(p, q)$. Set

$$
\mathcal{H}_{\mathcal{D}}^{n}(M, p)= \begin{cases}\mathcal{H}_{\mathbb{R}}^{n-1}(M)(p-1) \cap \underset{\substack{p^{\prime}+q^{\prime}=n-1 \\ p^{\prime}<p, q^{\prime}<p}}{\oplus} \mathcal{H}^{p^{\prime}, q^{\prime}}(M), & n<2 p, \\ \mathcal{H}_{\mathbb{R}}^{2 p}(M)(p) \cap \mathcal{H}^{p, p}(M), & n=2 p .\end{cases}
$$

Then the short exact sequence

$$
0 \rightarrow F^{p} H^{n-1}(M, \mathbb{C}) \rightarrow H^{n-1}(M, \mathbb{R}(p-1)) \rightarrow H_{\mathcal{D}}^{n}(M, \mathbb{R}(p)) \rightarrow 0
$$


for $n<2 p$ or the short exact sequence

$$
0 \rightarrow H_{\mathcal{D}}^{2 p}(M, \mathbb{R}(p)) \rightarrow F^{p} H^{2 p}(M, \mathbb{C}) \rightarrow H^{2 p}(M, \mathbb{R}(p-1)) \rightarrow 0
$$

yields an isomorphism

$$
H_{\mathcal{D}}^{n}(M, \mathbb{R}(p)) \simeq \mathcal{H}_{\mathcal{D}}^{n}(M, p)
$$

for $n \leq 2 p$.

Let us return to the arithmetic situation. An Arakelov variety is a pair $\bar{X}=\left(X, h_{X}\right)$ of an arithmetic variety $X$ and an $F_{\infty}$-invariant Kähler metric $h_{X}$ on $X(\mathbb{C})$. We now assume that $X$ is proper over $\mathbb{Z}$. Let $\mathcal{H}_{n}(X)$ denote the space of harmonic forms with respect to $h_{X}$ in $\mathcal{D}_{n}(X)$, that is,

$$
\mathcal{H}_{n}(X)=\underset{p}{\oplus} \mathcal{H}_{\mathcal{D}}^{2 p-n}(X(\mathbb{C}), p)^{\bar{F}_{\infty}^{*}=\mathrm{id}} .
$$

Then there is an isomorphism $H_{n}\left(\mathcal{D}_{*}(X), d_{\mathcal{D}}\right) \simeq \mathcal{H}_{n}(X)$, which implies the following:

Proposition 4.10. There is an orthogonal decomposition

$$
\operatorname{Ker} d_{\mathcal{D}}=\operatorname{Im} d_{\mathcal{D}} \oplus \mathcal{H}_{n}(X)
$$

in $\mathcal{D}_{n}(X)$.

Definition 4.11. The subgroup $K_{n}(\bar{X})=\left(\operatorname{ch}_{n}\right)^{-1}\left(\mathcal{H}_{n}(X)\right)$ of $\widehat{K}_{n}(X)$ is called the $n$-th Arakelov $K$-group of $\bar{X}=\left(X, h_{X}\right)$.

Theorem 4.12. There is an exact sequence

$$
K_{n+1}(X) \stackrel{\rho}{\rightarrow} \oplus_{p} H_{\mathcal{D}}^{2 p-n-1}(X, \mathbb{R}(p)) \rightarrow K_{n}(\bar{X}) \rightarrow K_{n}(X) \rightarrow 0 .
$$

Proof. This is derived from the fact that $[(0, \omega)] \in K_{n}(\bar{X})$ if and only if $d_{\mathcal{D}} \omega=0$, which follows from Proposition 4.10.

Künnemann has constructed a section of the inclusion from the Arakelov Chow group to the arithmetic Chow group in [12]. We adapt his method to the inclusion $K_{n}(\bar{X}) \hookrightarrow \widehat{K}_{n}(X)$. Let $\mathcal{H}: \mathcal{D}_{n}(X) \rightarrow \mathcal{H}_{n}(X)$ be the orthogonal projection with respect to the $L_{2}$-inner product. Let $(f, \omega)$ be a pair of a pointed cellular map $f: S^{n+1} \rightarrow|\widehat{S}(X)|$ and $\omega \in \widetilde{\mathcal{D}}_{n+1}(X)$. Then we can take $\omega_{\sharp} \in \widetilde{\mathcal{D}}_{n+1}(X)$ such that $\operatorname{ch}_{n}(f)-d_{\mathcal{D}} \omega_{\sharp}$ is harmonic and $\mathcal{H}\left(\omega_{\sharp}\right)=\mathcal{H}(\omega)$. Existence and uniqueness of $\omega_{\sharp}$ follow from Proposition 4.10. 
If $(f, \omega)$ is homotopy equivalent to $\left(f^{\prime}, \omega^{\prime}\right)$, then

$$
\operatorname{ch}_{n}\left(f^{\prime}\right)-d_{\mathcal{D}}\left(\omega^{\prime}-\omega_{\sharp}-\omega\right)=\operatorname{ch}_{n}(f)-d_{\mathcal{D}} \omega_{\sharp}
$$

and $\mathcal{H}\left(\omega^{\prime}-\omega_{\sharp}-\omega\right)=\mathcal{H}\left(\omega^{\prime}\right)$. Hence $\omega_{\sharp}^{\prime}=\omega^{\prime}-\omega_{\sharp}-\omega$, therefore $\left(f, \omega_{\sharp}\right)$ is homotopy equivalent to $\left(f^{\prime}, \omega_{\sharp}^{\prime}\right)$. Hence we can obtain a section

$$
\sigma: \widehat{K}_{n}(X) \rightarrow K_{n}(\bar{X})
$$

by $\sigma([(f, \omega)])=\left[\left(f, \omega_{\sharp}\right)\right]$. The map $\sigma$ is called the harmonic projection of $\widehat{K}_{n}(X)$.

\section{§5. A Product Formula for Higher Bott-Chern Forms}

\section{§5.1. A product formula}

We begin this section by recalling the multiplicative structure on $\mathcal{D}^{n}(M, p)$ for a compact complex algebraic manifold $M$ introduced in [4]. Let

$$
\text { - : } \mathcal{D}^{n}(M, p) \otimes \mathcal{D}^{m}(M, q) \rightarrow \mathcal{D}^{m+n}(M, p+q)
$$

be a homomorphism given by

$x \bullet y=(-1)^{n}\left(\partial x^{(p-1, n-p)}-\bar{\partial} x^{(n-p, p-1)}\right) \wedge y+x \wedge\left(\partial y^{(q-1, m-q)}-\bar{\partial} y^{(m-q, q-1)}\right)$

if $n<2 p$ and $m<2 q$ and $x \bullet y=x \wedge y$ if $n=2 p$ or $m=2 q$. Here $x^{(\alpha, \beta)}$ is the $(\alpha, \beta)$-part of the differential form $x$. Then it satisfies $d_{\mathcal{D}}(x \bullet y)=$ $d_{\mathcal{D}} x \bullet y+(-1)^{n} x \bullet d_{\mathcal{D}} y$ and $x \bullet y=(-1)^{n m} y \bullet x$. Moreover, it induces the product in the real Deligne cohomology defined in [1].

The higher Bott-Chern forms are not compatible with products, that is, $\operatorname{ch}_{n+m}(\mathcal{F} \otimes \mathcal{G})$ is not equal to $\operatorname{ch}_{n}(\mathcal{F}) \bullet \operatorname{ch}_{m}(\mathcal{G})$ in general. But since the Beilinson's regulator $K_{n}(M) \rightarrow H_{\mathcal{D}}^{2 p-n}(M, \mathbb{R}(p))$ respects the products, it is quite natural to expect that the difference $\operatorname{ch}_{n+m}(\mathcal{F} \otimes \mathcal{G})-\operatorname{ch}_{n}(\mathcal{F}) \bullet \operatorname{ch}_{m}(\mathcal{G})$ is written in terms of exact forms.

Let us introduce another operation on $\mathcal{D}^{n}(M, p)$. For integers $i$ and $j$ satisfying $1 \leq i \leq n$ and $1 \leq j \leq m$, let

$$
a_{i, j}^{n, m}=1-2\left(\begin{array}{c}
n+m \\
n
\end{array}\right)^{-1} \sum_{\alpha=0}^{i-1}\left(\begin{array}{c}
n+m-i-j+1 \\
n-\alpha
\end{array}\right)\left(\begin{array}{c}
i+j-1 \\
\alpha
\end{array}\right),
$$

where $\left(\begin{array}{l}a \\ b\end{array}\right)=\frac{(a+b) !}{a ! b !}$. When $b<0$ or $a<b,\left(\begin{array}{l}a \\ b\end{array}\right)$ is assumed to be zero. 
Lemma 5.1. We have $a_{i, j}^{n, m}=-a_{n-i+1, m-j+1}^{n, m}$ and $a_{i, j}^{n, m}=-a_{j, i}^{m, n}$.

Proof. Let us recall the following formula on binomial coefficients:

$$
\sum_{\alpha=0}^{a}\left(\begin{array}{c}
b \\
a-\alpha
\end{array}\right)\left(\begin{array}{c}
c \\
\alpha
\end{array}\right)=\left(\begin{array}{c}
b+c \\
a
\end{array}\right) .
$$

Using this identity, we have

$$
\begin{aligned}
a_{i, j}^{n, m}+a_{n-i+1, m-j+1}^{n, m}= & 2-2\left(\begin{array}{c}
n+m \\
n
\end{array}\right)-1 \sum_{\alpha=0}^{i-1}\left(\begin{array}{c}
n+m-i-j+1 \\
n-\alpha
\end{array}\right)\left(\begin{array}{c}
i+j-1 \\
\alpha
\end{array}\right) \\
& -2\left(\begin{array}{c}
n+m \\
n
\end{array}\right) \sum^{-1} \sum_{\alpha=0}^{n-i}\left(\begin{array}{c}
i+j-1 \\
n-\alpha
\end{array}\right)\left(\begin{array}{c}
n+m-i-j+1 \\
\alpha
\end{array}\right) \\
= & 2-2\left(\begin{array}{c}
n+m \\
n
\end{array}\right)^{-1} \sum_{\alpha=0}^{n}\left(\begin{array}{c}
n+m-i-j+1 \\
n-\alpha
\end{array}\right)\left(\begin{array}{c}
i+j-1 \\
\alpha
\end{array}\right) \\
= & 0 .
\end{aligned}
$$

Furthermore,

$$
\begin{aligned}
a_{j, i}^{m, n} & =1-2\left(\begin{array}{c}
n+m \\
m
\end{array}\right)-1 \\
-1 & \sum_{\alpha=0}^{j-1}\left(\begin{array}{c}
m+n-j-i+1 \\
m-\alpha
\end{array}\right)\left(\begin{array}{c}
j+i-1 \\
\alpha
\end{array}\right) \\
& =-1+2\left(\begin{array}{c}
n+m \\
m
\end{array}\right)^{-1} \sum_{\alpha=j}^{m}\left(\begin{array}{c}
m+n-j-i+1 \\
m-\alpha
\end{array}\right)\left(\begin{array}{c}
j+i-1 \\
\alpha
\end{array}\right) \\
& =-1+2\left(\begin{array}{c}
n+m \\
m
\end{array}\right)^{-1} \sum_{\alpha=j}^{m}\left(\begin{array}{c}
m+n-j-i+1 \\
n-j-i+1+\alpha
\end{array}\right)\left(\begin{array}{c}
j+i-1 \\
j+i-1-\alpha
\end{array}\right) .
\end{aligned}
$$

If we put $\beta=i+j-1-\alpha$, then

$$
\begin{aligned}
a_{j, i}^{m, n} & =-1+2\left(\begin{array}{c}
n+m \\
n
\end{array}\right)-1 \\
& =-a_{i, j}^{n, m} .
\end{aligned}
$$

For $x \in \mathcal{D}^{2 p-n}(M, p)$ and $y \in \mathcal{D}^{2 q-m}(M, q)$ with $n, m \geq 1$, we define another operation $x \Delta y$ as follows:

$$
x \Delta y=\sum_{\substack{1 \leq i \leq n \\ 1 \leq j \leq m}} a_{i, j}^{n, m} x^{(p-n+i-1, p-i)} \wedge y^{(q-m+j-1, q-j)} .
$$


If $n=0$ or $m=0$, then $x \Delta y$ is defined to be zero. The first claim of Lemma 5.1 implies that $x \Delta y \in \mathcal{D}^{2(p+q)-n-m-1}(M, p+q)$ and the second claim implies that $x \Delta y=(-1)^{n m+n+m} y \Delta x$.

Theorem 5.2. Let $\mathcal{F}($ resp. $\mathcal{G})$ be an exact hermitian $n$-cube (resp. $m$ cube) on $M$, then

$$
\begin{aligned}
\operatorname{ch}_{n+m}(\mathcal{F} \otimes \mathcal{G}) & -\operatorname{ch}_{n}(\mathcal{F}) \bullet \operatorname{ch}_{m}(\mathcal{G})=(-1)^{n+1} d_{\mathcal{D}}\left(\operatorname{ch}_{n}(\mathcal{F}) \Delta \operatorname{ch}_{m}(\mathcal{G})\right) \\
& +(-1)^{n} \operatorname{ch}_{n-1}(\partial \mathcal{F}) \Delta \operatorname{ch}_{m}(\mathcal{G})-\operatorname{ch}_{n}(\mathcal{F}) \Delta \operatorname{ch}_{m-1}(\partial \mathcal{G})
\end{aligned}
$$

\section{§5.2. Proof of Theorem 5.2}

Let us first prepare some notations. For differential forms $u_{1}, \ldots, u_{n}$ on $M$, let $\left(u_{1}, \ldots, u_{n}\right)^{(\alpha, \beta)}$ be the $(\alpha, \beta)$-part of $d u_{1} \wedge \cdots \wedge d u_{n}$. When $u_{i}$ is a $\left(p_{i}, p_{i}\right)$-form, let

$$
\left(u_{1}, \ldots, u_{n}\right)^{(i)}=\sum_{p}\left(u_{1}, \ldots, u_{n}\right)^{(p+i, p+n-i)}
$$

and

$$
S_{n}^{i}\left(u_{1}, \ldots, u_{n}\right)=(i-1) !(n-i) ! \sum_{\alpha=1}^{n}(-1)^{\alpha+1} u_{\alpha}\left(u_{1}, \ldots, \widehat{u_{\alpha}}, \ldots, u_{n}\right)^{(i-1)} .
$$

Then $S_{n}^{i}\left(u_{1}, \ldots, u_{n}\right) \in \mathcal{D}_{n}(M)$ if $u_{i} \in \mathcal{D}_{1}(M)$. If we take $u_{i}$ as $\log \left|z_{i}\right|^{2}$, $S_{n}^{i}\left(\log \left|z_{1}\right|^{2}, \ldots, \log \left|z_{n}\right|^{2}\right)$ is nothing but $S_{n}^{i}$ introduced in $\S 2.4$.

Lemma 5.3. If $u_{i}$ is a $\left(p_{i}, p_{i}\right)$-form on $M$, then

$$
\begin{aligned}
\partial S_{n}^{i}\left(u_{1}, \ldots, u_{n}\right)= & i !(n-i) !\left(u_{1}, \ldots, u_{n}\right)^{(i)} \\
& +(n-i) \sum_{\alpha=1}^{n}(-1)^{\alpha} \partial \bar{\partial} u_{\alpha} S_{n-1}^{i}\left(u_{1}, \ldots, \widehat{u_{\alpha}}, \ldots, u_{n}\right)
\end{aligned}
$$

and

$$
\begin{aligned}
\bar{\partial} S_{n}^{i}\left(u_{1}, \ldots, u_{n}\right)= & (i-1) !(n-i+1) !\left(u_{1}, \ldots, u_{n}\right)^{(i-1)} \\
& -(i-1) \sum_{\alpha=1}^{n}(-1)^{\alpha} \partial \bar{\partial} u_{\alpha} S_{n-1}^{i-1}\left(u_{1}, \ldots, \widehat{u_{\alpha}}, \ldots, u_{n}\right) .
\end{aligned}
$$

Proof. We have 


$$
\begin{aligned}
& \partial S_{n}^{i}\left(u_{1}, \ldots, u_{n}\right)=(i-1) !(n-i) ! \sum_{\alpha=1}^{n}(-1)^{\alpha+1} \partial\left(u_{\alpha}\left(u_{1}, \ldots, \widehat{u_{\alpha}}, \ldots, u_{n}\right)^{(i-1)}\right) \\
& =(i-1) !(n-i) ! \sum_{\alpha=1}^{n}(-1)^{\alpha+1} \partial u_{\alpha}\left(u_{1}, \ldots, \widehat{u_{\alpha}}, \ldots, u_{n}\right)^{(i-1)} \\
& +(i-1) !(n-i) ! \sum_{\alpha=1}^{n}(-1)^{\alpha+1} u_{\alpha} \\
& \quad \times\left(\sum_{\beta<\alpha}(-1)^{\beta-1} \partial \bar{\partial} u_{\beta}\left(u_{1}, \ldots, \widehat{u_{\beta}}, \ldots, \widehat{u_{\alpha}}, \ldots, u_{n}\right)^{(i-1)}\right. \\
& \left.\quad+\sum_{\alpha<\beta}(-1)^{\beta} \partial \bar{\partial} u_{\beta}\left(u_{1}, \ldots, \widehat{u_{\alpha}}, \ldots, \widehat{u_{\beta}}, \ldots, u_{n}\right)^{(i-1)}\right) \\
& +(i-1) !(n-i) ! \sum_{\beta=1}^{n}(-1)^{\beta} \partial \bar{\partial} u_{\beta} \\
& \quad \times\left(\sum_{\alpha<\beta}(-1)^{\alpha+1} u_{\alpha}\left(u_{1}, \ldots, \widehat{u_{\alpha}}, \ldots, \widehat{u_{\beta}}, \ldots, u_{n}\right)^{(i-1)}\right. \\
& +i !(n-i) !\left(u_{1}, \ldots, u_{n}\right)^{(i)}+(n-i) \sum_{\beta=1}^{n}(-1)^{\beta} \partial \bar{\partial} u_{\beta} S_{n-1}^{i}\left(u_{1}, \ldots, \widehat{u_{\beta}}, \ldots, u_{n}\right) . \\
& \left.+\sum_{\beta<\alpha}(-1)^{\alpha} u_{\alpha}\left(u_{1}, \ldots, \widehat{u_{\beta}}, \ldots, \widehat{u_{\alpha}}, \ldots, u_{n}\right)^{(i-1)}\right)
\end{aligned}
$$

The second identity can be proved in a similar way.

Lemma 5.4. For $\left(p_{i}, p_{i}\right)$-forms $u_{i}$ and $\left(q_{j}, q_{j}\right)$-forms $v_{j}$,

$$
\begin{aligned}
S_{n+m}^{k} & \left(u_{1}, \ldots, u_{n}, v_{1}, \ldots, v_{m}\right) \\
= & \sum_{i=1}^{k} \frac{(k-1) !(n+m-k) !}{(n-i) !(i-1) !} S_{n}^{i}\left(u_{1}, \ldots, u_{n}\right) \wedge\left(v_{1}, \ldots, v_{m}\right)^{(k-i)} \\
& \quad+(-1)^{n} \sum_{j=1}^{k} \frac{(k-1) !(n+m-k) !}{(m-j) !(j-1) !}\left(u_{1}, \ldots, u_{n}\right)^{(k-j)} \wedge S_{m}^{j}\left(v_{1}, \ldots, v_{m}\right) .
\end{aligned}
$$




\section{Hence}

$$
\begin{aligned}
& \sum_{k=1}^{n+m}(-1)^{k} S_{n+m}^{k}\left(u_{1}, \ldots, u_{n}, v_{1}, \ldots, v_{m}\right) \\
& \quad=\sum_{\substack{1 \leq i \leq n \\
0 \leq j \leq m}}(-1)^{i+j} \frac{(n+m-i-j) !(i+j-1) !}{(n-i) !(i-1) !} S_{n}^{i}\left(u_{1}, \ldots, u_{n}\right) \wedge\left(v_{1}, \ldots, v_{m}\right)^{(j)} \\
& \quad+\sum_{\substack{0 \leq i \leq n \\
1 \leq j \leq m}}(-1)^{n+i+j} \frac{(n+m-i-j) !(i+j-1) !}{(m-j) !(j-1) !}\left(v_{1}, \ldots, v_{n}\right)^{(i)} \wedge S_{m}^{j}\left(v_{1}, \ldots, v_{m}\right) .
\end{aligned}
$$

Proof. We have

$$
\begin{aligned}
S_{n+m}^{k}\left(u_{1}, \ldots, u_{n}, v_{1}, \ldots, v_{m}\right) & \\
= & (k-1) !(n+m-k) ! \sum_{\alpha=1}^{n}(-1)^{\alpha+1} u_{\alpha}\left(u_{1}, \ldots, \widehat{u_{\alpha}}, \ldots, u_{n}, v_{1}, \ldots, v_{m}\right)^{(k-1)} \\
& +(-1)^{n}(k-1) !(n+m-k) ! \sum_{\beta=1}^{n}(-1)^{\beta+1} v_{\beta} \\
& \times\left(u_{1}, \ldots, u_{n}, v_{1}, \ldots, \widehat{v_{\beta}}, \ldots, v_{m}\right)^{(k-1)} \\
= & (k-1) !(n+m-k) ! \sum_{\alpha=1}^{n}(-1)^{\alpha+1} u_{\alpha} \\
& \times\left(\sum_{i=1}^{k}\left(u_{1}, \ldots, \widehat{u_{\alpha}}, \ldots, u_{n}\right)^{(i-1)} \wedge\left(v_{1}, \ldots, v_{m}\right)^{(k-i)}\right) \\
& +(-1)^{n}(k-1) !(n+m-k) ! \sum_{\beta=1}^{n}(-1)^{\beta+1} v_{\beta} \\
& \quad \times\left(\sum_{j=1}^{k}\left(u_{1}, \ldots, u_{n}\right)^{(k-j)} \wedge\left(v_{1}, \ldots, \widehat{v_{\beta}}, \ldots, v_{m}\right)^{(j-1)}\right) \\
& +(-1)^{n} \sum_{j=1}^{k} \frac{(k-1) !(n+m-k) !}{(j-1) !(m-j) !}\left(u_{1}, \ldots, u_{n}\right)^{(k-j)} \wedge S_{m}^{j}\left(v_{1}, \ldots, v_{m}\right) . \\
= & \sum_{i=1}^{k} \frac{(k-1) !(n+m-k) !}{(i-1) !(n-i) !} S_{n}^{i}\left(u_{1}, \ldots, u_{n}\right) \wedge\left(v_{1}, \ldots, v_{m}\right)^{(k-i)} \\
& \\
& \\
& \\
& \\
& \\
& \\
&
\end{aligned}
$$


If we assume that $u_{i}$ and $v_{j}$ are in $\mathcal{D}_{1}(M)$, then by Lemma 5.3 we have

$$
\begin{aligned}
& d\left(\sum_{i=1}^{n}(-1)^{i} S_{n}^{i}\left(u_{1}, \ldots, u_{n}\right) \Delta \sum_{j=1}^{m}(-1)^{j} S_{m}^{j}\left(v_{1}, \ldots, v_{m}\right)\right) \\
& =\sum_{\substack{1 \leq i \leq n \\
1 \leq j \leq m}}(-1)^{i+j} a_{i, j}^{n, m} d S_{n}^{i}\left(u_{1}, \ldots, u_{n}\right) \wedge S_{m}^{j}\left(v_{1}, \ldots, v_{m}\right) \\
& +(-1)^{n+1} \sum_{\substack{1 \leq i \leq n \\
1 \leq j \leq m}}(-1)^{i+j} a_{i, j}^{n, m} S_{n}^{i}\left(u_{1}, \ldots, u_{n}\right) \wedge d S_{m}^{j}\left(v_{1}, \ldots, v_{m}\right) \\
& =\sum_{\substack{0 \leq i \leq n \\
1 \leq j \leq m}}(-1)^{i+j} i !(n-i) !\left(a_{i, j}^{n, m}-a_{i+1, j}^{n, m}\right)\left(u_{1}, \ldots, u_{n}\right)^{(i)} \wedge S_{m}^{j}\left(v_{1}, \ldots, v_{m}\right) \\
& +\sum_{\substack{1 \leq i \leq n-1 \\
1 \leq j \leq m}}(-1)^{i+j}\left((n-i) a_{i, j}^{n, m}+i a_{i+1, j}^{n, m}\right) \\
& \times\left(\sum_{\alpha=1}^{n}(-1)^{\alpha} \partial \bar{\partial} u_{\alpha} S_{n-1}^{i}\left(u_{1}, \ldots, \widehat{u_{\alpha}}, \ldots, u_{n}\right) \wedge S_{m}^{j}\left(v_{1}, \ldots, v_{m}\right)\right) \\
& +(-1)^{n+1} \sum_{\substack{1 \leq i \leq n \\
0 \leq j \leq m}}(-1)^{i+j} j !(m-j) !\left(a_{i, j}^{n, m}-a_{i, j+1}^{n, m}\right) \\
& \times S_{n}^{i}\left(u_{1}, \ldots, u_{n}\right) \wedge\left(v_{1}, \ldots, v_{m}\right)^{(j)} \\
& +(-1)^{n+1} \sum_{\substack{1 \leq i \leq n \\
1 \leq j \leq m-1}}(-1)^{i+j}\left((m-j) a_{i, j}^{n, m}+j a_{i, j+1}^{n, m}\right) \\
& \times\left(S_{n}^{i}\left(u_{1}, \ldots, u_{n}\right) \wedge \sum_{\beta=1}^{m}(-1)^{\beta} \partial \bar{\partial} v_{\beta} S_{m-1}^{j}\left(v_{1}, \ldots, \widehat{v_{\beta}}, \ldots, v_{m}\right)\right) .
\end{aligned}
$$

Let us compute the coefficients of the above expression. Since $\left(\begin{array}{c}n+m-i-j+1 \\ n-\alpha\end{array}\right)=$ $\left(\begin{array}{c}n+m-i-j \\ n-\alpha\end{array}\right)+\left(\begin{array}{c}n+m-i-j \\ n-1-\alpha\end{array}\right)$ and $\left(\begin{array}{c}i+j \\ \alpha\end{array}\right)=\left(\begin{array}{c}i+j-1 \\ \alpha\end{array}\right)+\left(\begin{array}{c}i+j-1 \\ \alpha-1\end{array}\right)$,

$$
\begin{aligned}
a_{i, j}^{n, m}-a_{i+1, j}^{n, m}= & 1-2\left(\begin{array}{c}
n+m \\
n
\end{array}\right) \sum_{\alpha=0}^{-1}\left(\begin{array}{c}
n+m-i-j+1 \\
n-\alpha
\end{array}\right)\left(\begin{array}{c}
i+j-1 \\
\alpha
\end{array}\right) \\
& -1+2\left(\begin{array}{c}
n+m \\
n
\end{array}\right) \sum_{\alpha=0}^{-1}\left(\begin{array}{c}
n+m-i-j \\
n-\alpha
\end{array}\right)\left(\begin{array}{c}
i+j \\
\alpha
\end{array}\right) \\
= & 2\left(\begin{array}{c}
n+m \\
n
\end{array}\right)^{-1}\left(\begin{array}{c}
n+m-i-j \\
n-i
\end{array}\right)\left(\begin{array}{c}
i+j-1 \\
i
\end{array}\right)
\end{aligned}
$$


for $1 \leq i \leq n-1$ and

$$
\begin{aligned}
a_{i, j}^{n, m}-a_{i, j+1}^{n, m}= & 1-2\left(\begin{array}{c}
n+m \\
n
\end{array}\right)^{-1} \sum_{\alpha=0}^{i-1}\left(\begin{array}{c}
n+m-i-j+1 \\
n-\alpha
\end{array}\right)\left(\begin{array}{c}
i+j-1 \\
\alpha
\end{array}\right) \\
& -1+2\left(\begin{array}{c}
n+m \\
n
\end{array}\right)-1 \sum_{\alpha=0}^{i-1}\left(\begin{array}{c}
n+m-i-j \\
n-\alpha
\end{array}\right)\left(\begin{array}{c}
i+j \\
\alpha
\end{array}\right) \\
= & -2\left(\begin{array}{c}
n+m \\
n
\end{array}\right)^{-1}\left(\begin{array}{c}
n+m-i-j \\
n-i
\end{array}\right)\left(\begin{array}{c}
i+j-1 \\
i-1
\end{array}\right) \\
= & -2\left(\begin{array}{c}
n+m \\
n
\end{array}\right)^{-1}\left(\begin{array}{c}
n+m-i-j \\
m-j
\end{array}\right)\left(\begin{array}{c}
i+j-1 \\
j
\end{array}\right)
\end{aligned}
$$

for $1 \leq j \leq m-1$. It follows from the definition of $a_{i, j}^{n, m}$ and Lemma 5.1 that

$$
\begin{aligned}
& a_{1, j}^{n, m}=1-2\left(\begin{array}{c}
n+m \\
n
\end{array}\right)^{-1}\left(\begin{array}{c}
n+m-j \\
n
\end{array}\right), \\
& a_{n, j}^{n, m}=-1+2\left(\begin{array}{c}
n+m \\
n
\end{array}\right)^{-1}\left(\begin{array}{c}
n+j-1 \\
n
\end{array}\right), \\
& a_{i, 1}^{n, m}=-1+2\left(\begin{array}{c}
n+m \\
m
\end{array}\right)^{-1}\left(\begin{array}{c}
n+m-i \\
m
\end{array}\right), \\
& a_{i, m}^{n, m}=1-2\left(\begin{array}{c}
n+m \\
m
\end{array}\right)-1\left(\begin{array}{c}
m+i-1 \\
m
\end{array}\right) .
\end{aligned}
$$

Moreover, by Lemma A.1 we have

$$
\begin{aligned}
& \left(\begin{array}{l}
n \\
-
\end{array}\right) a_{i, j}^{n, m}+i a_{i+1, j}^{n, m} \\
& \quad=n-2\left(\begin{array}{c}
n+m \\
n
\end{array}\right)-1 \\
& \left.\quad(n-i) \sum_{\alpha=0}^{i-1}\left(\begin{array}{c}
n+m-i-j+1 \\
n-\alpha
\end{array}\right)\left(\begin{array}{c}
i+j-1 \\
\alpha
\end{array}\right)+i \sum_{\alpha=0}^{i}\left(\begin{array}{c}
n+m-i-j \\
n-\alpha
\end{array}\right)\left(\begin{array}{c}
i+j \\
\alpha
\end{array}\right)\right) \\
& \quad=n-2 n\left(\begin{array}{c}
n+m-1 \\
n-1
\end{array}\right)-1 \sum_{\alpha=0}^{i-1}\left(\begin{array}{c}
n+m-i-j \\
n-1-\alpha
\end{array}\right)\left(\begin{array}{c}
i+j-1 \\
\alpha
\end{array}\right) \\
& \quad=n a_{i, j}^{n-1, m}
\end{aligned}
$$

and

$$
\begin{aligned}
& (m-j) a_{i, j}^{n, m}+j a_{i, j+1}^{n, m} \\
& =m-2\left(\begin{array}{c}
n+m \\
n
\end{array}\right)^{-1}\left((m-j) \sum_{\alpha=0}^{i-1}\left(\begin{array}{c}
n+m-i-j+1 \\
n-\alpha
\end{array}\right)\left(\begin{array}{c}
i+j-1 \\
\alpha
\end{array}\right)+j \sum_{\alpha=0}^{i-1}\left(\begin{array}{c}
n+m-i-j \\
n-\alpha
\end{array}\right)\left(\begin{array}{c}
i+j \\
\alpha
\end{array}\right)\right) \\
& =m-2 m\left(\begin{array}{c}
n+m-1 \\
n
\end{array}\right)^{-1} \sum_{\alpha=0}^{i-1}\left(\begin{array}{c}
n+m-i-j \\
n-\alpha
\end{array}\right)\left(\begin{array}{c}
i+j-1 \\
\alpha
\end{array}\right) \\
& =m a_{i, j}^{n, m-1} \text {. }
\end{aligned}
$$


These computations imply that

$$
\begin{aligned}
& d\left(\sum_{i=1}^{n}(-1)^{i} S_{n}^{i}\left(u_{1}, \ldots, u_{n}\right) \Delta \sum_{j=1}^{n}(-1)^{j} S_{m}^{j}\left(v_{1}, \ldots, v_{m}\right)\right) \\
& =2\left(\begin{array}{c}
n+m \\
n
\end{array}\right)^{-1} \sum_{\substack{0 \leq i \leq n \\
1 \leq j \leq m}}(-1)^{i+j} i !(n-i) !\left(\begin{array}{c}
n+m-i-j \\
n-i
\end{array}\right)\left(\begin{array}{c}
i+j-1 \\
i
\end{array}\right) \\
& \times\left(u_{1}, \ldots, u_{n}\right)^{(i)} \wedge S_{m}^{j}\left(v_{1}, \ldots, v_{m}\right) \\
& +n \sum_{\substack{1 \leq i \leq n-1 \\
1 \leq j \leq m}}(-1)^{i+j} a_{i, j}^{n-1, m} \sum_{\alpha=1}^{n}(-1)^{\alpha} \partial \bar{\partial} u_{\alpha} \\
& \times S_{n-1}^{i}\left(u_{1}, \ldots, \widehat{u_{\alpha}}, \ldots, u_{n}\right) \wedge S_{m}^{j}\left(v_{1}, \ldots, v_{m}\right) \\
& +2(-1)^{n}\left(\begin{array}{c}
n+m \\
n
\end{array}\right)^{-1} \sum_{\substack{1 \leq i \leq n \\
0 \leq j \leq m}}(-1)^{i+j} j !(m-j) !\left(\begin{array}{c}
n+m-i-j \\
m-j
\end{array}\right)\left(\begin{array}{c}
i+j-1 \\
j
\end{array}\right) \\
& \times S_{n}^{i}\left(u_{1}, \ldots, u_{n}\right) \wedge\left(v_{1}, \ldots, v_{m}\right)^{(j)} \\
& +(-1)^{n+1} m \sum_{\substack{1 \leq i \leq n \\
1 \leq j \leq m-1}}(-1)^{i+j} a_{i, j}^{n, m-1} S_{n}^{i}\left(u_{1}, \ldots, u_{n}\right) \\
& \wedge \sum_{\beta=1}^{m}(-1)^{\beta} \partial \bar{\partial} v_{\beta} S_{m-1}^{j}\left(v_{1}, \ldots, \widehat{v_{\beta}}, \ldots, v_{m}\right) \\
& -\sum_{1 \leq j \leq m}(-1)^{j} n !\left(u_{1}, \ldots, u_{n}\right)^{(0)} \wedge S_{m}^{j}\left(v_{1}, \ldots, v_{m}\right) \\
& -\sum_{1 \leq j \leq m}(-1)^{n+j} n !\left(u_{1}, \ldots, u_{n}\right)^{(n)} \wedge S_{m}^{j}\left(v_{1}, \ldots, v_{m}\right) \\
& -\sum_{1 \leq i \leq n}(-1)^{n+i} m ! S_{n}^{i}\left(u_{1}, \ldots, u_{n}\right) \wedge\left(v_{1}, \ldots, v_{m}\right)^{(0)} \\
& -\sum_{1 \leq i \leq n}(-1)^{n+m+i} m ! S_{n}^{i}\left(u_{1}, \ldots, u_{n}\right) \wedge\left(v_{1}, \ldots, v_{m}\right)^{(m)} \\
& =2\left(\begin{array}{c}
n+m \\
n
\end{array}\right)^{-1} \sum_{\substack{0 \leq i \leq n \\
1 \leq j \leq m}}(-1)^{i+j} \frac{(n+m-i-j) !(i+j-1) !}{(m-j) !(j-1) !} \\
& \times\left(u_{1}, \ldots, u_{n}\right)^{(i)} \wedge S_{m}^{j}\left(v_{1}, \ldots, v_{m}\right) \\
& +n \sum_{\substack{1 \leq i \leq n-1 \\
1 \leq j \leq m}}(-1)^{i+j} a_{i, j}^{n-1, m} \sum_{\alpha=1}^{n}(-1)^{\alpha} \partial \bar{\partial} u_{\alpha}
\end{aligned}
$$




$$
\begin{gathered}
\quad \times S_{n-1}^{i}\left(u_{1}, \ldots, \widehat{u_{\alpha}}, \ldots, u_{n}\right) \wedge S_{m}^{j}\left(v_{1}, \ldots, v_{m}\right) \\
+2(-1)^{n}\left(\begin{array}{c}
n+m \\
n
\end{array}\right) \sum_{\substack{1 \leq i \leq n \\
0 \leq j \leq m}}(-1)^{i+j} \frac{(n+m-i-j) !(i+j-1) !}{(n-i) !(i-1) !} \\
\quad \times S_{n}^{i}\left(u_{1}, \ldots, u_{n}\right) \wedge\left(v_{1}, \ldots, v_{m}\right)^{(j)} \\
+(-1)^{n+1} m \sum_{\substack{1 \leq i \leq n \\
1 \leq j \leq m-1}}(-1)^{i+j} a_{i, j}^{n, m-1} S_{n}^{i}\left(u_{1}, \ldots, u_{n}\right) \\
\wedge \sum_{\beta=1}^{m}(-1)^{\beta} \partial \bar{\partial} v_{\beta} S_{m-1}^{j}\left(v_{1}, \ldots, \widehat{v_{\beta}}, \ldots, v_{m}\right) \\
-\sum_{1 \leq j \leq m}(-1)^{j}\left(\bar{\partial} S_{n}^{1}\left(u_{1}, \ldots, u_{n}\right)+(-1)^{n} \partial S_{n}^{n}\left(u_{1}, \ldots, u_{n}\right)\right) \wedge S_{m}^{j}\left(v_{1}, \ldots, v_{m}\right) \\
-\sum_{1 \leq i \leq n}(-1)^{n+i} S_{n}^{i}\left(u_{1}, \ldots, u_{n}\right) \wedge\left(\bar{\partial} S_{m}^{1}\left(v_{1}, \ldots, v_{m}\right)+(-1)^{m} \partial S_{m}^{m}\left(v_{1}, \ldots, v_{m}\right)\right) .
\end{gathered}
$$

Applying Lemma 5.4 to the above, we can obtain the following:

Proposition 5.5. For $u_{i} \in \mathcal{D}_{1}(M)$ and $v_{j} \in \mathcal{D}_{1}(M)$, we have

$$
\begin{aligned}
& d\left(\sum_{i=1}^{n}(-1)^{i} S_{n}^{i}\left(u_{1}, \ldots, u_{n}\right) \Delta \sum_{j=1}^{m}(-1)^{j} S_{m}^{j}\left(v_{1}, \ldots, v_{m}\right)\right) \\
& =2(-1)^{n}\left(\begin{array}{c}
n+m \\
n
\end{array}\right)-1 \sum_{k=1}^{n+m}(-1)^{k} S_{n+m}^{k}\left(u_{1}, \ldots, u_{n}, v_{1}, \ldots, v_{m}\right) \\
& +n \sum_{\substack{1 \leq i \leq n-1 \\
1 \leq j \leq m}}(-1)^{i+j} a_{i, j}^{n-1, m} \sum_{\alpha=1}^{n}(-1)^{\alpha} \partial \bar{\partial} u_{\alpha} \\
& \quad \times S_{n-1}^{i}\left(u_{1}, \ldots, \widehat{u_{\alpha}}, \ldots, u_{n}\right) \wedge S_{m}^{j}\left(v_{1}, \ldots, v_{m}\right) \\
& +(-1)^{n+1} m \sum_{1 \leq i \leq n}(-1)^{i+j} a_{i, j}^{n, m-1} S_{n}^{i}\left(u_{1}, \ldots, u_{n}\right) \\
& \wedge \sum_{\beta=1}^{m}(-1)^{\beta} \partial \bar{\partial} v_{\beta} S_{m-1}^{j}\left(v_{1}, \ldots, \widehat{v_{\beta}}, \ldots, v_{m}\right) \\
& +(-1)^{n+1}\left(\sum_{i=1}^{n}(-1)^{i} S_{n}^{i}\left(u_{1}, \ldots, u_{n}\right)\right) \bullet\left(\sum_{j=1}^{m}(-1)^{j} S_{m}^{j}\left(v_{1}, \ldots, v_{m}\right)\right) .
\end{aligned}
$$

Let us return to the proof of Theorem 5.2. We may assume that $\mathcal{F}$ and $\mathcal{G}$ are emi-cubes. For $s<t$, let $\pi_{1}:\left(\mathbb{P}^{1}\right)^{t} \rightarrow\left(\mathbb{P}^{1}\right)^{s}$ denote the projection 
given by $\left(x_{1}, \ldots, x_{t}\right) \mapsto\left(x_{1}, \ldots, x_{s}\right)$ and let $\pi_{2}:\left(\mathbb{P}^{1}\right)^{t} \rightarrow\left(\mathbb{P}^{1}\right)^{s}$ denote the projection given by $\left(x_{1}, \ldots, x_{t}\right) \mapsto\left(x_{t-s+1}, \ldots, x_{t}\right)$. Let $u_{i}=\log \left|z_{i}\right|^{2}$ for $i=$ $1, \ldots, n$ and $v_{j}=\log \left|z_{n+j}\right|^{2}$ for $j=1, \ldots, m$. If we regard $\partial \bar{\partial} \log \left|z_{i}\right|^{2}$ as $-2 \pi \sqrt{-1}\left(\delta_{\left\{z_{i}=0\right\}}-\delta_{\left\{z_{i}=\infty\right\}}\right)$, then the above identity is still valid as currents on $\left(\mathbb{P}^{1}\right)^{n}$. Hence we have

$$
\begin{aligned}
& d_{\mathcal{D}}\left(\operatorname{ch}_{n}(\mathcal{F}) \Delta \operatorname{ch}_{m}(\mathcal{G})\right)=-d\left(\operatorname{ch}_{n}(\mathcal{F}) \Delta \operatorname{ch}_{m}(\mathcal{G})\right) \\
& =\frac{(-1)^{n+m+1}}{4 n ! m !(2 \pi \sqrt{-1})^{n+m}} \int_{\left(\mathbb{P}^{1}\right)^{n+m}} \pi_{1}^{*} \operatorname{ch}_{0}\left(\operatorname{tr}_{n} \mathcal{F}\right) \wedge \pi_{2}^{*} \operatorname{ch}_{0}\left(\operatorname{tr}_{m} \mathcal{G}\right) \\
& \wedge d\left(\left(\sum_{i=1}^{n}(-1)^{i} \pi_{1}^{*} S_{n}^{i}\right) \triangle\left(\sum_{j=1}^{m}(-1)^{j} \pi_{2}^{*} S_{m}^{j}\right)\right) \\
& =\frac{(-1)^{m+1}}{2(n+m) !(2 \pi \sqrt{-1})^{n+m}} \int_{\left(\mathbb{P}^{1}\right)^{n+m}} \operatorname{ch}_{0}\left(\operatorname{tr}_{n+m}(\mathcal{F} \otimes \mathcal{G})\right) \wedge \sum_{k=1}^{n+m}(-1)^{k} S_{n+m}^{k} \\
& +\frac{(-1)^{n+m+1}}{4(n-1) ! m !(2 \pi \sqrt{-1})^{n+m-1}} \int_{\left(\mathbb{P}^{1}\right)^{n+m-1}} \operatorname{ch}_{0}\left(\operatorname{tr}_{n+m-1}(\partial \mathcal{F} \otimes \mathcal{G})\right) \\
& \wedge \sum_{\substack{1 \leq i \leq n-1 \\
1 \leq j \leq m}}(-1)^{i+j} a_{i, j}^{n-1, m} \pi_{1}^{*} S_{n-1}^{i} \wedge \pi_{2}^{*} S_{m}^{j} \\
& +\frac{(-1)^{m}}{4 n !(m-1) !(2 \pi \sqrt{-1})^{n+m-1}} \int_{\left(\mathbb{P}^{1}\right)^{n+m-1}} \operatorname{ch}_{0}\left(\operatorname{tr}_{n+m-1}(\mathcal{F} \otimes \partial \mathcal{G})\right) \\
& \wedge \sum_{\substack{1 \leq i \leq n \\
1 \leq j \leq m-1}}(-1)^{i+j} a_{i, j}^{n, m-1} \pi_{1}^{*} S_{n}^{i} \wedge \pi_{2}^{*} S_{m-1}^{j} \\
& +\frac{(-1)^{m}}{4 n ! m !(2 \pi \sqrt{-1})^{n+m}} \int_{\left(\mathbb{P}^{1}\right)^{n+m}} \operatorname{ch}_{0}\left(\operatorname{tr}_{n+m}(\mathcal{F} \otimes \mathcal{G})\right) \\
& \wedge\left(\left(\sum_{i=1}^{n}(-1)^{i} \pi_{1}^{*} S_{n}^{i}\right) \bullet\left(\sum_{j=1}^{m}(-1)^{j} \pi_{2}^{*} S_{m}^{j}\right)\right) \\
& =(-1)^{n+1} \operatorname{ch}_{n+m}(\mathcal{F} \otimes \mathcal{G})+\operatorname{ch}_{n-1}(\partial \mathcal{F}) \Delta \operatorname{ch}_{m}(\mathcal{G}) \\
& +(-1)^{n+1} \operatorname{ch}_{n}(\mathcal{F}) \Delta \operatorname{ch}_{m}(\partial \mathcal{G})+(-1)^{n} \operatorname{ch}_{n}(\mathcal{F}) \bullet \operatorname{ch}_{m}(\mathcal{G}) \text {. }
\end{aligned}
$$

\section{$\S 6 . \quad$ Product}

\section{$\S 6.1$. Notations on bisimplicial sets}

A bisimplicial set is a contravariant functor from the category of pairs of finite ordered sets to the category of sets. The product $S \times T$ and the reduced 
product $S \wedge T$ of two simplicial sets $S, T$ are examples of bisimplicial sets. The topological realization $|S|$ of a bisimplicial set $S$ is defined in a similar way to that of a simplicial set.

For a bisimplicial set $S$, let $\Delta(S)$ denote the simplicial set given by $[n] \mapsto$ $S([n],[n])$. Then its topological realization $|\Delta(S)|$ is a subdivision of $|S|$. Hence the identity map $|S| \rightarrow|\Delta(S)|$ is cellular, although the inverse is not.

\section{§6.2. Product in higher $K$-theory}

In this subsection we review the product in higher algebraic $K$-theory by means of the $S$-construction [15]. For a small exact category $\mathfrak{A}$, let $S_{n} S_{m} \mathfrak{A}$ be the set of functors

$$
E: \operatorname{Ar}[n] \times \operatorname{Ar}[m] \rightarrow \mathfrak{A},(i \leq j, \alpha \leq \beta) \mapsto E_{(i, j) \times(\alpha, \beta)}
$$

satisfying the following conditions:

(1) $E_{(i, i) \times(\alpha, \beta)}=0$ and $E_{(i, j) \times(\alpha, \alpha)}=0$.

(2) For any $i \leq j \leq k$ and $\alpha \leq \beta, E_{(i, j) \times(\alpha, \beta)} \rightarrow E_{(i, k) \times(\alpha, \beta)} \rightarrow E_{(j, k) \times(\alpha, \beta)}$ is a short exact sequence of $\mathfrak{A}$.

(3) For any $i \leq j$ and $\alpha \leq \beta \leq \gamma, E_{(i, j) \times(\alpha, \beta)} \rightarrow E_{(i, j) \times(\alpha, \gamma)} \rightarrow E_{(i, j) \times(\beta, \gamma)}$ is a short exact sequence of $\mathfrak{A}$.

Then $([n],[m]) \mapsto S_{n} S_{m} \mathfrak{A}$ is a bisimplicial set. Let us denote it by $S^{(2)} \mathfrak{A}$. The natural identification $S_{1} S_{m} \mathfrak{A}=S_{m} \mathfrak{A}$ yields a map of bisimplicial sets

$$
S^{1} \wedge S \mathfrak{A} \rightarrow S^{(2)} \mathfrak{A},
$$

and its adjoint map $|S \mathfrak{A}| \rightarrow \Omega\left|S^{(2)} \mathfrak{A}\right|$ is proved to be a homotopy equivalence.

When $\mathfrak{A}$ is equipped with tensor product, we can define a map of bisimplicial sets

$$
m: S \mathfrak{A} \wedge S \mathfrak{A} \rightarrow S^{(2)} \mathfrak{A}
$$

by $m(E, F)_{(i, j) \times(\alpha, \beta)}=E_{i, j} \otimes F_{\alpha, \beta}$. This induces a pairing

$$
m_{*}: \pi_{n+1}(|S \mathfrak{A}|) \times \pi_{m+1}(|S \mathfrak{A}|) \rightarrow \pi_{n+m+2}\left(\left|S^{(2)} \mathfrak{A}\right|\right) .
$$

Combining this with the isomorphisms $K_{n}(\mathfrak{A}) \simeq \pi_{n+1}(|S \mathfrak{A}|) \simeq \pi_{n+2}\left(\left|S^{(2)} \mathfrak{A}\right|\right)$ yields the product in higher algebraic $K$-theory $K_{*}(\mathfrak{A})$. 


\section{$\S 6.3 . \quad G$-construction}

In [8], Gillet and Grayson have constructed a simplicial set $G \mathfrak{A}$ associated with a small exact category $\mathfrak{A}$ that is homotopy equivalent to the loop space of the $S$-construction $S \mathfrak{A}$. In this subsection we recall their construction.

Let $G_{n} \mathfrak{A}$ be the set of pairs $\left(E^{+}, E^{-}\right)$of $E^{+}, E^{-} \in S_{n+1} \mathfrak{A}$ with $\partial_{0} E^{+}=$ $\partial_{0} E^{-}$. Then $[n] \mapsto G_{n} \mathfrak{A}$ becomes a simplicial set by $\partial_{k}\left(E^{+}, E^{-}\right)=\left(\partial_{k+1} E^{+}\right.$, $\left.\partial_{k+1} E^{-}\right)$and $s_{k}\left(E^{+}, E^{-}\right)=\left(s_{k+1} E^{+}, s_{k+1} E^{-}\right)$. We fix $0=(0,0) \in G_{0} \mathfrak{A}$ as the base point of $G \mathfrak{A}$.

Let $\Delta[1]$ be the simplicial set represented by [1]. Let $\iota_{k}$ denote the element of $\Delta[1]_{n}$ given by

$$
\iota_{k}(i)= \begin{cases}0, & i<k, \\ 1, & i \geq k .\end{cases}
$$

Then $\Delta[1]_{n}=\left\{\iota_{0}, \iota_{1}, \ldots, \iota_{n+1}\right\}$. Let

$$
\chi_{n}^{ \pm}: \Delta[1]_{n} \times G_{n} \mathfrak{A} \rightarrow S_{n} \mathfrak{A}
$$

be the maps given by

$$
\chi_{n}^{ \pm}\left(\iota_{k},\left(E^{+}, E^{-}\right)\right)= \begin{cases}\partial_{0} E^{ \pm}, & k=0 \\ \left(s_{0}\right)^{k-1}\left(\partial_{1}\right)^{k} E^{ \pm}, & k \geq 1 .\end{cases}
$$

Then $\chi^{ \pm}=\left\{\chi_{n}^{ \pm}\right\}: \Delta(\Delta[1] \times G \mathfrak{A}) \rightarrow S \mathfrak{A}$ are maps of simplicial sets such that $\chi^{ \pm}(\{0\} \times G \mathfrak{A})=*$ and $\left.\chi^{+}\right|_{\{1\} \times G \mathfrak{A}}=\left.\chi^{-}\right|_{\{1\} \times G \mathfrak{A}}$.

Let $T^{1}$ be the simplicial set given by the following cocartesian square:

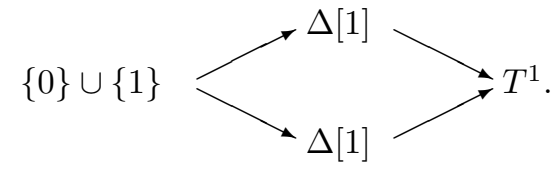

We fix 0 as the base point of $T^{1}$. The topological realization of $T^{1}$ is the barycentric subdivision of the circle $S^{1}=I / \partial I$. Gluing the maps $\chi^{ \pm}$, we obtain a map of simplicial sets

$$
\chi: \Delta\left(T^{1} \wedge G \mathfrak{A}\right) \rightarrow S \mathfrak{A} .
$$

It is the main theorem of [8] that the adjoint map $|G \mathfrak{A}| \rightarrow \Omega|S \mathfrak{A}|$ to $|\chi|$ is a homotopy equivalence. Therefore we have an isomorphism $\pi_{i}(|G \mathfrak{A}|, 0) \simeq$ $K_{i}(\mathfrak{A})$. 
We next introduce a description of the product in $K$-theory by means of $G$-construction. Let

$$
\begin{aligned}
G_{n} G_{m} \mathfrak{A}=\left\{\left(E^{++}, E^{+-}, E^{-+}, E^{--}\right) ; E^{ \pm \pm} \in S_{n+1} S_{m+1} \mathfrak{A}\right. \\
\\
\left.\partial_{0} E^{+ \pm}=\partial_{0} E^{- \pm}, \partial_{0}^{\prime} E^{ \pm+}=\partial_{0}^{\prime} E^{ \pm-}\right\}
\end{aligned}
$$

where $\partial_{0}$ is the boundary map on the first factor of the bisimplicial set $S^{(2)} \mathfrak{A}$ and $\partial_{0}^{\prime}$ is the boundary map on the second factor. Then $([n],[m]) \mapsto G_{n} G_{m} \mathfrak{A}$ becomes a bisimplicial set. Let us denote it by $G^{(2)} \mathfrak{A}$. Let $R: G_{n} \mathfrak{A} \rightarrow G_{0} G_{n} \mathfrak{A}$ be the map given by $R\left(E^{+}, E^{-}\right)=\left(E^{+}, E^{-}, 0,0\right)$. Then it is shown in [8] that $R$ induces a homotopy equivalence $R: G \mathfrak{A} \rightarrow G^{(2)} \mathfrak{A}$.

Let us define a map of bisimplicial sets

$$
m^{G}: G \mathfrak{A} \wedge G \mathfrak{A} \rightarrow G^{(2)} \mathfrak{A}
$$

by $m^{G}(E, F)^{ \pm \pm}=E^{ \pm} \otimes F^{ \pm}$for $E=\left(E^{+}, E^{-}\right) \in G_{n} \mathfrak{A}$ and $F=\left(F^{+}, F^{-}\right) \in$ $G_{m} \mathfrak{A}$. Then the pairing

$$
m_{*}^{G}: \pi_{n}(|G \mathfrak{A}|, 0) \times \pi_{m}(|G \mathfrak{A}|, 0) \rightarrow \pi_{n+m}\left(\left|G^{(2)} \mathfrak{A}\right|, 0\right)
$$

induces the product in $K_{*}(\mathfrak{A})$.

Finally, let us define an exact cube associated with an element of $G \mathfrak{A}$ or $G^{(2)} \mathfrak{A}$. The map $\chi$ yields a homomorphism of chain complexes

$$
\mathrm{Cub}: C_{*}(|G \mathfrak{A}|) \stackrel{\chi_{*}}{\longrightarrow} C_{*}(|S \mathfrak{A}|)[-1] \stackrel{\mathrm{Cub}}{\longrightarrow} \mathrm{Cub}_{*}(\mathfrak{A}) .
$$

Let us define $\operatorname{Cub}(E) \in \operatorname{Cub}_{n}(\mathfrak{A})$ associated with $E=\left(E^{+}, E^{-}\right) \in G_{n} \mathfrak{A}$ as the image of $[E] \in C_{*}(|G \mathfrak{A}|)$ by the above map. In other words, $\operatorname{Cub}(E)=$ $\operatorname{Cub}\left(E^{+}\right)-\operatorname{Cub}\left(E^{-}\right)$. Similarly, we define an exact $(n+m)$-cube associated with $E=\left(E^{ \pm \pm}\right) \in G_{n} G_{m} \mathfrak{A}$ by

$$
\operatorname{Cub}(E)=\operatorname{Cub}\left(E^{++}\right)-\operatorname{Cub}\left(E^{+-}\right)-\operatorname{Cub}\left(E^{-+}\right)+\operatorname{Cub}\left(E^{--}\right),
$$

where $\operatorname{Cub}\left(E^{ \pm \pm}\right)$is the image of the element of $E^{ \pm \pm} \in S_{n+1} S_{m+1} \mathfrak{A}$ by the homomorphism

$$
S_{n+1} S_{m+1} \mathfrak{A} \rightarrow \operatorname{Cub}_{n}\left(S_{m+1} \mathfrak{A}\right) \rightarrow \operatorname{Cub}_{n}\left(\operatorname{Cub}_{m}(\mathfrak{A})\right)=\operatorname{Cub}_{n+m}(\mathfrak{A}) .
$$

When $E=\left(E^{ \pm \pm}\right)$is degenerate, the associated cube $\operatorname{Cub}(E)$ is zero in $\operatorname{Cub}_{*}(\mathfrak{A})$ by Lemma 4.1. Hence $E=\left(E^{ \pm \pm}\right) \mapsto \operatorname{Cub}(E)$ induces a homomorphism

$\mathrm{Cub}: C_{*}\left(\left|G^{(2)} \mathfrak{A}\right|\right) \rightarrow \mathrm{Cub}_{*}(\mathfrak{A})$. 
Proposition 6.1. The following diagram is commutative:

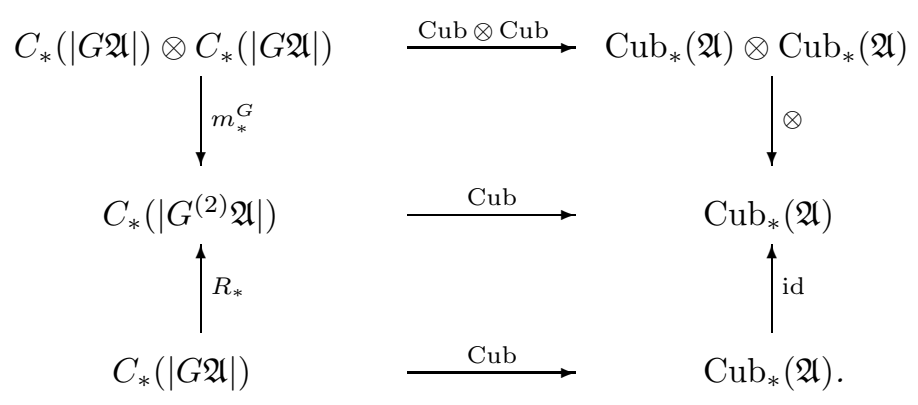

§6.4. Pairing $\widehat{\mathcal{K}}_{0} \times \widehat{K}_{n} \rightarrow \widehat{K}_{n}$

For a proper arithmetic variety $X$, let $\widehat{G}(X)=G(\widehat{\mathcal{P}}(X))$, the $G$-construction of the category of hermitian vector bundles on $X$. Then there is a homomorphism of chain complexes

$$
\operatorname{ch}: C_{*}(|\widehat{G}(X)|) \stackrel{\mathrm{Cub}}{\longrightarrow} \widehat{\mathrm{Cub}}_{*}(X) \stackrel{\mathrm{ch}}{\longrightarrow} \mathcal{D}_{*}(X) .
$$

Proposition 6.2. The map $\chi: \Delta\left(T^{1} \wedge \widehat{G}(X)\right) \rightarrow \widehat{S}(X)$ yields an isomorphism

$$
\widehat{\chi}_{*}: \widehat{\pi}_{n}(|\widehat{G}(X)|, \mathrm{ch}) \simeq \widehat{\pi}_{n+1}(|\widehat{S}(X)|, \mathrm{ch})
$$

by $[(f, \omega)] \mapsto[(\chi(1 \wedge f),-\omega)]$. Hence for $n \geq 1$ there is a canonical isomorphism

$$
\widehat{K}_{n}(X) \simeq \widehat{\pi}_{n}(|\widehat{G}(X)|, \mathrm{ch}) .
$$

Proof. It is obvious that the map $(f, \omega) \mapsto(\chi(1 \wedge f),-\omega)$ gives rise to a homomorphism of the modified homotopy groups. Consider the following commutative diagram:

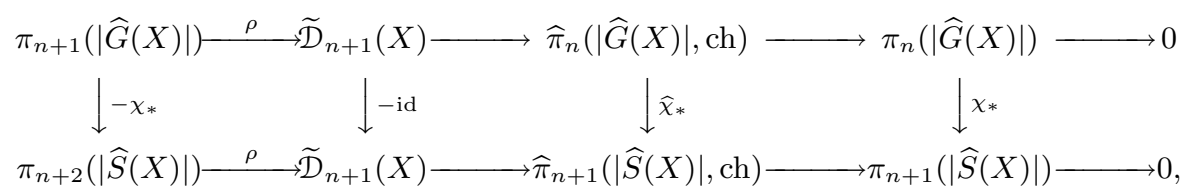

where the upper and lower sequences are exact by Theorem 3.3. Hence the proposition follows from the five lemma.

If we set $\widehat{G}^{(2)}(X)=G^{(2)}(\widehat{\mathcal{P}}(X))$, then we have $\operatorname{ch}: C_{*}\left(\left|\widehat{G}^{(2)}(X)\right|\right) \stackrel{\mathrm{Cub}}{\longrightarrow} \widehat{\mathrm{Cub}}_{*}(X) \stackrel{\mathrm{ch}}{\longrightarrow} \mathcal{D}_{*}(X)$ 
and the following square is commutative by Proposition 6.1:

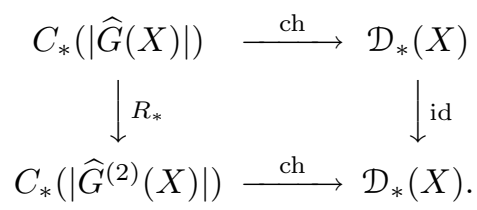

Hence $R$ induces an isomorphism

$$
\widehat{R}_{*}: \widehat{\pi}_{n}(|\widehat{G}(X)|, \mathrm{ch}) \simeq \widehat{\pi}_{n}\left(\left|\widehat{G}^{(2)}(X)\right|, \mathrm{ch}\right) .
$$

The product $\widehat{\mathcal{K}}_{0}(X) \times \widehat{\mathcal{K}}_{0}(X) \rightarrow \widehat{\mathcal{K}}_{0}(X)$ given in $[9]$ is written as follows:

$$
[(\bar{E}, \omega)] \times[(\bar{F}, \tau)]=\left[\left(\bar{E} \otimes \bar{F}, \operatorname{ch}_{0}(\bar{E}) \bullet \tau+\omega \bullet \operatorname{ch}_{0}(\bar{F})+\omega \bullet d_{\mathcal{D}} \tau\right)\right],
$$

and it makes $\widehat{\mathcal{K}}_{0}(X)$ a commutative associative algebra. To construct product in higher arithmetic $K$-theory, we will use the $G$-construction. However, since we have not had any expression of $\widehat{K}_{0}(X)$ by means of the $G$-construction, we have to distinguish the cases including $\widehat{K}_{0}(X)$ from the general case.

Let $(\bar{E}, \eta)$ be a pair of a hermitian vector bundle $\bar{E}$ on $X$ and $\eta \in \widetilde{\mathcal{D}}_{1}(X)$ and let $(f, \omega)$ be a pair of a pointed cellular map $f: S^{n} \rightarrow|\widehat{G}(X)|$ and $\omega \in$ $\widetilde{\mathcal{D}}_{n+1}(X)$. Let us define a product of these pairs by

$$
(\bar{E}, \eta) \times(f, \omega)=\left(\bar{E} \otimes f, \operatorname{ch}_{0}(\bar{E}) \bullet \omega+\eta \bullet \operatorname{ch}_{n}(f)+\eta \bullet d_{\mathcal{D}} \omega\right),
$$

where $\bar{E} \otimes f: S^{n} \stackrel{f}{\longrightarrow}|\widehat{G}(X)| \stackrel{\bar{E} \otimes}{\longrightarrow}|\widehat{G}(X)|$.

Theorem 6.3. The above product gives rise to a pairing

$$
\times: \widehat{\mathcal{K}}_{0}(X) \times \widehat{K}_{n}(X) \rightarrow \widehat{K}_{n}(X) .
$$

Proof. To prove the theorem, we have to show that $(\bar{E}, \eta) \times(f, \omega)$ is compatible with the equivalence relations for $\widehat{\mathcal{K}}_{0}(X)$ and $\widehat{K}_{n}(X)$. Let us first show the compatibility with the relation for $\widehat{K}_{n}(X)$.

Let $H:\left(S^{n} \times I\right) /(\{*\} \times I) \rightarrow|\widehat{G}(X)|$ be a cellular homotopy from $(f, \omega)$ to $\left(f^{\prime}, \omega^{\prime}\right)$. We write $\operatorname{ch}_{n+1}(H)$ for $\operatorname{ch} \circ H_{*}\left(\left[S^{n} \times I\right]\right) \in \mathcal{D}_{n+1}(X)$. Then $\omega^{\prime}-\omega=$ $(-1)^{n+1} \operatorname{ch}_{n+1}(H)$ and the map

$$
\bar{E} \otimes H:\left(S^{n} \times I\right) /(\{*\} \times I) \stackrel{H}{\rightarrow}|\widehat{G}(X)| \stackrel{\bar{E} \otimes}{\longrightarrow}|\widehat{G}(X)|
$$

is a cellular homotopy from $\bar{E} \otimes f$ to $\bar{E} \otimes f^{\prime}$. Furthermore, by Proposition 5.2 we have

$$
\operatorname{ch}_{n+1}(\bar{E} \otimes H)=\operatorname{ch}_{0}(\bar{E}) \bullet \operatorname{ch}_{n+1}(H)=(-1)^{n+1} \operatorname{ch}_{0}(\bar{E}) \bullet\left(\omega^{\prime}-\omega\right) .
$$


This tells that $\bar{E} \otimes H$ is a cellular homotopy from $(\bar{E}, \eta) \times(f, \omega)$ to $(\bar{E}, \eta) \times$ $\left(f^{\prime}, \omega^{\prime}\right)$.

Next we show the compatibility with the relation for $\widehat{\mathcal{K}}_{0}(X)$. Let $\mathcal{E}: 0 \rightarrow$ $\bar{E} \rightarrow \bar{F} \rightarrow \bar{G} \rightarrow 0$ be a short exact sequence of hermitian vector bundles on $X$. Consider the following 1-dimensional subcomplex of $|\widehat{G}(X)|$ :

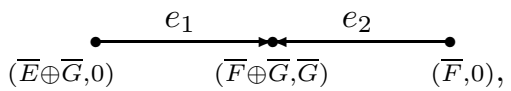

where

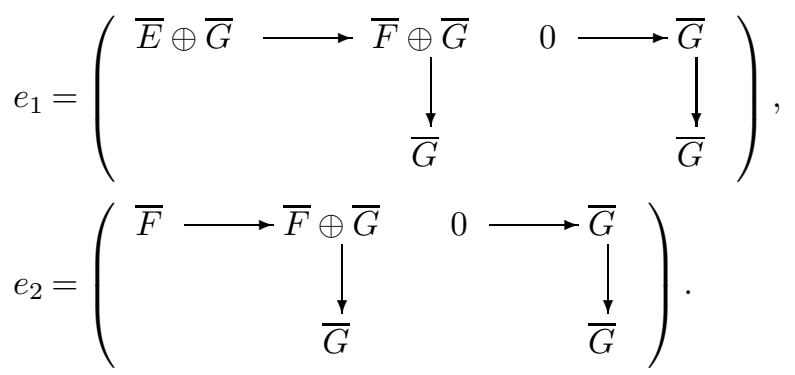

We denote by $\iota_{\mathcal{E}}: I \rightarrow|\widehat{G}(X)|$ a cellular map such that $\iota_{\mathcal{E}}(I)=e_{1} e_{2}^{-1}$.

For a pointed cellular map $f: S^{n} \rightarrow|\widehat{G}(X)|$, let

$H:\left(S^{n} \times I\right) /(\{*\} \times I) \stackrel{T}{\rightarrow}\left(I \times S^{n}\right) /(I \times\{*\}) \stackrel{\iota \varepsilon \wedge f}{\longrightarrow}|\widehat{G}(X)| \wedge|\widehat{G}(X)| \stackrel{m^{G}}{\longrightarrow}\left|\widehat{G}^{(2)}(X)\right|$,

where $T(s, t)=(t, s)$ for $t \in S^{n}$ and $s \in I$. If $H_{0}(s)=H(s, 0)$, then $H_{0}$ is written as

$$
S^{n} \stackrel{f}{\longrightarrow}|\widehat{G}(X)| \stackrel{\iota \bar{E} \oplus \bar{G}^{\wedge i d}}{\longrightarrow}|\widehat{G}(X)| \wedge|\widehat{G}(X)| \stackrel{m^{G}}{\longrightarrow}\left|\widehat{G}^{(2)}(X)\right|,
$$

where $\iota_{\bar{E} \oplus \bar{G}}: S^{0} \rightarrow|\widehat{G}(X)|$ is the pointed map determined by $(\bar{E} \oplus \bar{G}, 0) \in$ $\widehat{G}_{0}(X)$. Since the diagram

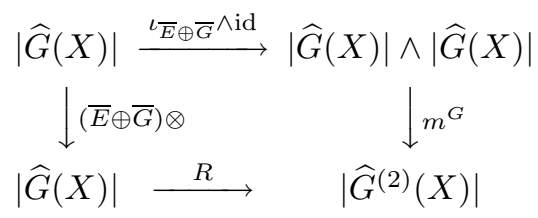

is commutative, we have $H_{0}=R((\bar{E} \oplus \bar{G}) \otimes f)$. If $H_{1}(s)=H(s, 1)$, then we can show that $H_{1}=R(\bar{F} \otimes f)$ in the same way. Moreover, Proposition 5.2 implies 
that

$$
\begin{aligned}
\operatorname{ch}_{n+1}(H) & =(-1)^{n} \operatorname{ch}_{n+1}\left(m_{*}^{G}\left(\iota_{\mathcal{E}} \wedge f\right)_{*}\left(\left[I \times S^{n}\right]\right)\right) \\
& \equiv(-1)^{n} \operatorname{ch}_{1}\left(\iota_{\varepsilon}\right) \bullet \operatorname{ch}_{n}(f) \\
& =(-1)^{n} \operatorname{ch}_{1}(\varepsilon) \bullet \operatorname{ch}_{n}(f)
\end{aligned}
$$

modulo $\operatorname{Im} d_{\mathcal{D}}$. Hence $H$ is a cellular homotopy from $\left(R((\bar{E} \oplus \bar{G}) \otimes f), \operatorname{ch}_{1}(\mathcal{E}) \bullet\right.$ $\left.\operatorname{ch}_{n}(f)\right)$ to $(R(\bar{F} \otimes f), 0)$. Since $\widehat{R}_{*}: \widehat{\pi}_{n}(|\widehat{G}(X)|, \mathrm{ch}) \rightarrow \widehat{\pi}_{n}\left(\left|\widehat{G}^{(2)}(X)\right|, \mathrm{ch}\right)$ is bijective,

$$
\left[\left((\bar{E} \oplus \bar{G}) \otimes f, \operatorname{ch}_{1}(\varepsilon) \bullet \operatorname{ch}_{n}(f)\right)\right]=[(\bar{F} \otimes f, 0)]
$$

in $\widehat{\pi}_{n}(|\widehat{G}(X)|, \mathrm{ch})$.

The short exact sequence $\mathcal{E}$ gives the relation

$$
[(\bar{E}, 0)]+[(\bar{G}, 0)]=\left[\left(\bar{F},-\operatorname{ch}_{1}(\mathcal{E})\right)\right]
$$

in $\widehat{\mathcal{K}}_{0}(X)$. We have

$[(\bar{E}, 0) \times(f, \omega)] \cdot[(\bar{G}, 0) \times(f, \omega)]=\left[\left((\bar{E} \otimes f) \cdot(\bar{G} \otimes f),\left(\operatorname{ch}_{0}(\bar{E})+\operatorname{ch}_{0}(\bar{G})\right) \bullet \omega\right)\right]$

and

$$
\begin{aligned}
{[(\bar{F},} & \left.\left.-\operatorname{ch}_{1}(\mathcal{E})\right) \times(f, \omega)\right] \\
& =\left[\left(\bar{F} \otimes f, \operatorname{ch}_{0}(\bar{F}) \bullet \omega-\operatorname{ch}_{1}(\mathcal{E}) \bullet \operatorname{ch}_{n}(f)-d_{\mathcal{D}} \operatorname{ch}_{1}(\mathcal{E}) \bullet \omega\right)\right] \\
& =\left[\left((\bar{E} \oplus \bar{G}) \otimes f,\left(\operatorname{ch}_{0}(\bar{E})+\operatorname{ch}_{0}(\bar{G})\right) \bullet \omega\right)\right]
\end{aligned}
$$

in $\widehat{\pi}_{n}(|\widehat{G}(X)|$, ch). Hence Theorem 6.3 follows from Lemma 6.4 and Lemma 6.5 below.

Lemma 6.4. For a pointed cellular map $f: S^{n} \rightarrow|\widehat{G}(X)|$ and two hermitian vector bundles $\bar{E}, \bar{G}$ on $X$,

$$
[((\bar{E} \otimes f) \oplus(\bar{G} \otimes f), 0)]=[((\bar{E} \otimes f) \cdot(\bar{G} \otimes f), 0)]
$$

in $\widehat{\pi}_{n}(|\widehat{G}(X)|, \mathrm{ch})$.

Proof. Let us first describe the map $(\bar{E} \otimes f) \oplus(\bar{G} \otimes f)$ explicitly. Since $f$ is a pointed cellular map, the map

$$
S^{n} \stackrel{\Delta}{\hookrightarrow} S^{n} \times S^{n} \stackrel{(\bar{E} \otimes f) \times(\bar{G} \otimes f)}{\longrightarrow}|\Delta(\widehat{G}(X) \times \widehat{G}(X))|
$$

is also a pointed cellular map. Moreover, the direct sum of hermitian vector bundles induces a map of simplicial sets $\oplus: \Delta(\widehat{G}(X) \times \widehat{G}(X)) \rightarrow \widehat{G}(X)$. Then 
the map $(\bar{E} \otimes f) \oplus(\bar{G} \otimes f)$ is expressed as the composition of these two cellular maps, that is,

$(\bar{E} \otimes f) \oplus(\bar{G} \otimes f): S^{n} \stackrel{\Delta}{\hookrightarrow} S^{n} \times S^{n} \stackrel{(\bar{E} \otimes f) \times(\bar{G} \otimes f)}{\longrightarrow}|\Delta(\widehat{G}(X) \times \widehat{G}(X))| \stackrel{\oplus}{\longrightarrow}|\widehat{G}(X)|$.

Consider the homomorphism of chain complexes

$$
\operatorname{ch} \oplus \operatorname{ch}: C_{*}(|\Delta(\widehat{G}(X) \times \widehat{G}(X))|) \rightarrow \mathcal{D}_{*}(X) \oplus \mathcal{D}_{*}(X)
$$

given by $(E, F) \mapsto\left(\operatorname{ch}_{n}(E), \operatorname{ch}_{n}(F)\right)$ for $E, F \in \widehat{G}_{n}(X)$ and the inclusion

$$
i n_{1}\left(\text { resp. } i n_{2}\right): \widehat{G}(X) \rightarrow \Delta(\widehat{G}(X) \times \widehat{G}(X))
$$

given by $i n_{1}(t)=(t, *)$ (resp. $i n_{2}(t)=(*, t)$ ). Then we have the following commutative diagram:

$$
\begin{array}{ccc}
C_{*}(|\widehat{G}(X)|) & \text { ch } & \mathcal{D}_{*}(X) \\
\left\lfloor i n_{1_{*}}\left(\text { resp. } i n_{2 *}\right)\right. & & \\
C_{*}(|\Delta(\widehat{G}(X) \times \widehat{G}(X))|) \stackrel{\operatorname{ch} \oplus \mathrm{ch}}{\longrightarrow} \mathcal{D}_{*}(X) \oplus \mathcal{D}_{*}(X),
\end{array}
$$

where the right vertical arrow is $i n_{1}(\omega)=(\omega, 0)\left(\right.$ resp. $\left.i n_{2}(\omega)=(0, \omega)\right)$. On the other hand, the projection

$$
p r_{1}\left(\text { resp. } p r_{2}\right): \Delta(\widehat{G}(X) \times \widehat{G}(X)) \rightarrow \widehat{G}(X)
$$

given by $p r_{1}(x, y)=x$ (resp. $p r_{2}(x, y)=y$ ) is also a map of simplicial sets and we have the following commutative diagram:

$$
\begin{aligned}
& C_{*}(|\Delta(\widehat{G}(X) \times \widehat{G}(X))|) \stackrel{\operatorname{ch} \oplus \operatorname{ch}}{\longrightarrow} \mathcal{D}_{*}(X) \oplus \mathcal{D}_{*}(X) \\
& \left.\downarrow p r_{1 *}\left(\text { resp. } p r_{2 *}\right) \quad \downarrow p r_{1} \text { (resp. } p r_{2}\right) \\
& C_{*}(|\widehat{G}(X)|) \quad \stackrel{\mathrm{ch}}{\longrightarrow} \quad \mathcal{D}_{*}(X),
\end{aligned}
$$

where the right vertical arrow is $\operatorname{pr}_{1}(\omega, \tau)=\omega\left(\right.$ resp. $\left.p r_{2}(\omega, \tau)=\tau\right)$. Hence we have four homomorphisms between the modified homotopy groups

$$
\widehat{\pi}_{n}(|\Delta(\widehat{G}(X) \times \widehat{G}(X))|, \operatorname{ch} \oplus \mathrm{ch}) \underset{\widehat{i n_{j_{*}}}}{\stackrel{\widehat{p r}_{j_{*}}}{\rightleftarrows}} \widehat{\pi}_{n}(|\widehat{G}(X)|, \mathrm{ch})
$$

that induce an isomorphism

$$
\widehat{\pi}_{n}(|\widehat{G}(X)|, \mathrm{ch}) \oplus \widehat{\pi}_{n}(|\widehat{G}(X)|, \mathrm{ch}) \simeq \widehat{\pi}_{n}(|\Delta(\widehat{G}(X) \times \widehat{G}(X))|, \operatorname{ch} \oplus \mathrm{ch})
$$


by $(x, y) \mapsto \widehat{i n_{1 *}}(x) \cdot{\widehat{i n_{2}}}(y)$. The inverse of it is $\widehat{p r}_{1 *} \oplus \widehat{p r_{2 *}}$. Since

$$
\begin{aligned}
& {\widehat{p r_{1}}}([(((\bar{E} \otimes f) \times(\bar{G} \otimes f)) \Delta, 0)])=[(\bar{E} \otimes f, 0)], \\
& {\widehat{p r_{2}}}_{2}([(((\bar{E} \otimes f) \times(\bar{G} \otimes f)) \Delta, 0)])=[(\bar{G} \otimes f, 0)],
\end{aligned}
$$

we have

$$
[(((\bar{E} \otimes f) \times(\bar{G} \otimes f)) \Delta, 0)]=\widehat{i n_{1 *}}([(\bar{E} \otimes f, 0)]) \cdot \widehat{i n_{2 *}}([(\bar{G} \otimes f, 0)])
$$

in $\widehat{\pi}_{*}(|\Delta(\widehat{G}(X) \times \widehat{G}(X))|$, ch $\oplus \mathrm{ch})$.

The commutative diagram

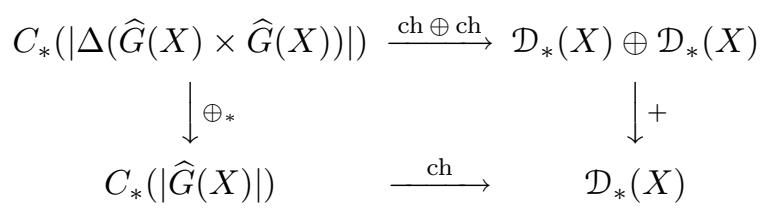

implies a homomorphism

$$
\widehat{\oplus}_{*}: \widehat{\pi}_{n}(|\Delta(\widehat{G}(X) \times \widehat{G}(X))|, \operatorname{ch} \oplus \operatorname{ch}) \rightarrow \widehat{\pi}_{n}(|\widehat{G}(X)|, \mathrm{ch}) .
$$

Since $\widehat{\oplus}_{*} \widehat{i n}_{j}$ is the identity homomorphism, we have

$$
\begin{aligned}
{[((\bar{E} \otimes f) \oplus(\bar{G} \otimes f), 0)] } & =\widehat{\oplus}_{*}([(((\bar{E} \otimes f) \times(\bar{G} \otimes f)) \Delta, 0)]) \\
& =[(\bar{E} \otimes f, 0)] \cdot[(\bar{G} \otimes f, 0)] \\
& =[((\bar{E} \otimes f) \cdot(\bar{G} \otimes f), 0)] .
\end{aligned}
$$

Lemma 6.5. In the same notations as in Lemma 6.4, we have

$$
[((\bar{E} \oplus \bar{G}) \otimes f, 0)]=[((\bar{E} \otimes f) \oplus(\bar{G} \otimes f), 0)]
$$

in $\widehat{\pi}_{n}(|\widehat{G}(X)|, \mathrm{ch})$.

Proof. Consider the following diagram:

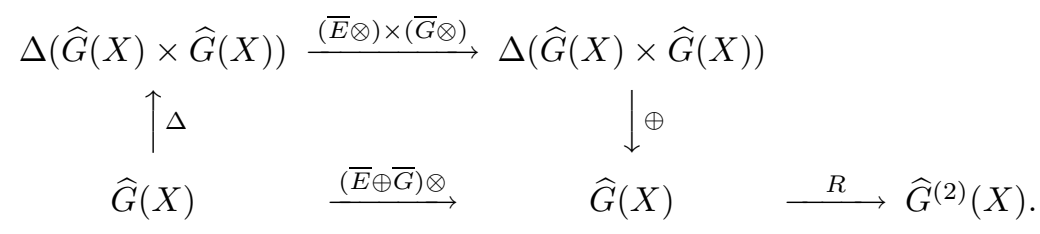


Let $\alpha_{0}: \widehat{G}(X) \rightarrow \widehat{G}^{(2)}(X)$ be the upper map of the diagram and $\alpha_{1}$ the lower map. Then for $P=\left(P^{ \pm}\right) \in \widehat{G}_{n}(X), \alpha_{0}(P)$ and $\alpha_{1}(P)$ are elements of $\widehat{G}_{0} \widehat{G}_{n}(X)$ written as follows:

$$
\begin{gathered}
\alpha_{0}(P)=\left(\left(\bar{E} \otimes P^{+}\right) \oplus\left(\bar{G} \otimes P^{+}\right),\left(\bar{E} \otimes P^{-}\right) \oplus\left(\bar{G} \otimes P^{-}\right), 0,0\right), \\
\alpha_{1}(P)=\left((\bar{E} \oplus \bar{G}) \otimes P^{+},(\bar{E} \oplus \bar{G}) \otimes P^{-}, 0,0\right) .
\end{gathered}
$$

The canonical isometries $\left(\bar{E} \otimes P^{ \pm}\right) \oplus\left(\bar{G} \otimes P^{ \pm}\right) \simeq(\bar{E} \oplus \bar{G}) \otimes P^{ \pm}$give an element of $\widehat{G}_{1} \widehat{G}_{n}(X)$ whose Bott-Chern form is zero. Collecting these elements for all $P=\left(P^{ \pm}\right)$provides a map of bisimplicial sets $\Psi: \Delta[1] \times \widehat{G}(X) \rightarrow \widehat{G}^{(2)}(X)$ such that $\Psi(0, s)=\alpha_{0}(s)$ and $\Psi(1, s)=\alpha_{1}(s)$. Therefore for any pointed cellular $\operatorname{map} f: S^{n} \rightarrow|\widehat{G}(X)|$,

$$
\begin{aligned}
H:\left(S^{n} \times I\right) /(\{*\} \times I) & \stackrel{T}{\longrightarrow}\left(I \times S^{n}\right) /(I \times\{*\}) \stackrel{\operatorname{id} \times f}{\longrightarrow}(I \times|\widehat{G}(X)|) /(I \times\{*\}) \\
& \stackrel{|\Psi|}{\longrightarrow}\left|\widehat{G}^{(2)}(X)\right|
\end{aligned}
$$

is a cellular homotopy from $R((\bar{E} \otimes f) \oplus(\bar{G} \otimes f))$ to $R((\bar{E} \oplus \bar{G}) \otimes f)$ such that $\operatorname{ch}_{n+1}(H)=0$. Since $\widehat{R}_{*}: \widehat{\pi}_{n}(|\widehat{G}(X)|, \mathrm{ch}) \rightarrow \widehat{\pi}_{n}\left(\left|\widehat{G}^{(2)}(X)\right|\right.$, ch $)$ is bijective, we have

$$
[((\bar{E} \otimes f) \oplus(\bar{G} \otimes f), 0)]=[((\bar{E} \oplus \bar{G}) \otimes f, 0)] .
$$

We can define a pairing $\widehat{K}_{n}(X) \times \widehat{\mathcal{K}}_{0}(X) \rightarrow \widehat{K}_{n}(X)$ by

$$
[(f, \omega)] \times[(\bar{E}, \eta)]=\left[\left(f \otimes \bar{E},(-1)^{n} \operatorname{ch}_{n}(f) \bullet \eta+\omega \bullet \operatorname{ch}_{0}(\bar{E})+\omega \bullet d_{\mathcal{D}} \eta\right)\right],
$$

where $f \otimes \bar{E}: S^{n} \stackrel{f}{\longrightarrow}|\widehat{G}(X)| \stackrel{\otimes \bar{E}}{\longrightarrow}|\widehat{G}(X)|$. Combining these pairings with the isomorphism $\widehat{\alpha}: \widehat{\mathcal{K}}_{0}(X) \simeq \widehat{K}_{0}(X)$, we can obtain a pairing

$$
\times: \widehat{K}_{n}(X) \times \widehat{K}_{m}(X) \rightarrow \widehat{K}_{n+m}(X)
$$

when $n=0$ or $m=0$.

\section{$\S 6.5$. Pairing of higher arithmetic $K$-theory}

In this subsection we define a pairing $\widehat{K}_{n}(X) \times \widehat{K}_{m}(X) \rightarrow \widehat{K}_{n+m}(X)$ in the case of $n, m \geq 1$. For two pointed cellular maps $f: S^{n} \rightarrow|\widehat{G}(X)|$ and $g: S^{m} \rightarrow|\widehat{G}(X)|$, let

$$
f \times g: S^{n+m}=S^{n} \wedge S^{m} \stackrel{f \wedge g}{\longrightarrow}|\widehat{G}(X)| \wedge|\widehat{G}(X)| \stackrel{m^{G}}{\longrightarrow}\left|\widehat{G}^{(2)}(X)\right| .
$$


For $\omega \in \widetilde{\mathcal{D}}_{n+1}(X)$ and $\tau \in \widetilde{\mathcal{D}}_{m+1}(X)$, a product of pairs $(f, \omega)$ and $(g, \tau)$ is defined by

$$
\begin{aligned}
& (f, \omega) \times(g, \tau) \\
& =\left(f \times g,(-1)^{n} \operatorname{ch}_{n}(f) \bullet \tau+\omega \bullet \operatorname{ch}_{m}(g)+\omega \bullet d_{\mathcal{D}} \tau+(-1)^{n} \operatorname{ch}_{n}(f) \triangle \operatorname{ch}_{m}(g)\right) .
\end{aligned}
$$

Proposition 6.6. The above product gives rise to a pairing

$$
\widehat{m}_{*}^{G}: \widehat{\pi}_{n}(|\widehat{G}(X)|, \mathrm{ch}) \times \widehat{\pi}_{m}(|\widehat{G}(X)|, \mathrm{ch}) \rightarrow \widehat{\pi}_{n+m}\left(\left|\widehat{G}^{(2)}(X)\right|, \mathrm{ch}\right) .
$$

Proof. For a cellular homotopy $H$ from $(f, \omega)$ to $\left(f^{\prime}, \omega^{\prime}\right)$, let $\widetilde{H}$ be a cellular map given by

$$
\widetilde{H}:\left(S^{n+m} \times I\right) /(\{*\} \times I) \longrightarrow|\widehat{G}(X)| \wedge|\widehat{G}(X)| \stackrel{m^{G}}{\longrightarrow}\left|\widehat{G}^{(2)}(X)\right|,
$$

where the first map is $\left(s_{1}, s_{2}, t\right) \mapsto\left(H\left(s_{1}, t\right), g\left(s_{2}\right)\right)$ for $s_{1} \in S^{n}, s_{2} \in S^{m}$ and $t \in I$. Then $\widetilde{H}$ is a homotopy from $f \times g$ to $f^{\prime} \times g$. Theorem 5.2 and Proposition 6.1 imply that

$$
\begin{aligned}
\operatorname{ch}_{n+m+1}(\widetilde{H}) \\
=(-1)^{m} \operatorname{ch}_{n+m+1}\left(m_{*}^{G}(H \times g)_{*}\left(\left[S^{n} \times I \times S^{m}\right]\right)\right) \\
\equiv(-1)^{m} \operatorname{ch}_{n+1}(H) \bullet \operatorname{ch}_{m}(g)+(-1)^{n+m+1} \operatorname{ch}_{n}\left(\partial H_{*}\left(\left[S^{n} \times I\right]\right)\right) \Delta \operatorname{ch}_{m}(g) \\
=(-1)^{n+m+1}\left(\omega^{\prime}-\omega\right) \bullet \operatorname{ch}_{m}(g)+(-1)^{m+1}\left(\operatorname{ch}_{n}\left(f^{\prime}\right)-\operatorname{ch}_{n}(f)\right) \Delta \operatorname{ch}_{m}(g) \\
=(-1)^{n+m+1}\left(\omega^{\prime} \bullet \operatorname{ch}_{m}(g)+(-1)^{n} \operatorname{ch}_{n}\left(f^{\prime}\right) \Delta \operatorname{ch}_{m}(g)\right) \\
\quad-(-1)^{n+m+1}\left(\omega \bullet \operatorname{ch}_{m}(g)+(-1)^{n} \operatorname{ch}_{n}(f) \Delta \operatorname{ch}_{m}(g)\right)
\end{aligned}
$$

modulo $\operatorname{Im} d_{\mathcal{D}}$. This tells that the map $\widetilde{H}$ is a cellular homotopy from $(f, \omega) \times$ $(g, \tau)$ to $\left(f^{\prime}, \omega^{\prime}\right) \times(g, \tau)$.

If $H^{\prime}$ is a cellular homotopy from $(g, \tau)$ to $\left(g^{\prime}, \tau^{\prime}\right)$, we can show in the same way that the map

$$
\left(S^{n+m} \times I\right) /(\{*\} \times I) \stackrel{f \wedge H^{\prime}}{\longrightarrow}|\widehat{G}(X)| \wedge|\widehat{G}(X)| \stackrel{m^{G}}{\longrightarrow}\left|\widehat{G}^{(2)}(X)\right|
$$

is a cellular homotopy from $(f, \omega) \times(g, \tau)$ to $(f, \omega) \times\left(g^{\prime}, \tau^{\prime}\right)$.

Definition 6.7. For $n, m \geq 1$, we define a product in higher arithmetic $K$-theory

$$
\times: \widehat{K}_{n}(X) \times \widehat{K}_{m}(X) \rightarrow \widehat{K}_{n+m}(X)
$$

by the following homomorphism:

$$
\begin{aligned}
& \widehat{\pi}_{n}(|\widehat{G}(X)|, \mathrm{ch}) \times \widehat{\pi}_{m}(|\widehat{G}(X)|, \mathrm{ch}) \stackrel{\widehat{m}_{*}^{G}}{\longrightarrow} \widehat{\pi}_{n+m}\left(\left|\widehat{G}^{(2)}(X)\right|, \mathrm{ch}\right) \\
& \stackrel{\widehat{R}_{*}^{-1}}{\longrightarrow} \widehat{\pi}_{n+m}(|\widehat{G}(X)|, \mathrm{ch}) .
\end{aligned}
$$


Proposition 6.8. The Chern form map respects the products, that is, we have

$$
\operatorname{ch}_{n+m}(x \times y)=\operatorname{ch}_{n}(x) \bullet \operatorname{ch}_{m}(y)
$$

for $x \in \widehat{K}_{n}(X)$ and $y \in \widehat{K}_{m}(X)$.

Proof. Assume $n, m \geq 1$. Define the Chern form map on $\widehat{\pi}_{n+m}\left(\left|\widehat{G}^{(2)}(X)\right|\right.$, ch) by

$$
\operatorname{ch}_{n+m}([(f, \omega)])=\operatorname{ch}_{n+m}\left(f_{*}\left(\left[S^{n+m}\right]\right)\right)+d_{\mathcal{D}} \omega \in \mathcal{D}_{n+m}(X) .
$$

Then $\operatorname{ch}_{n+m}\left(\widehat{R}_{*}(x)\right)=\operatorname{ch}_{n+m}(x)$ for any $x \in \widehat{\pi}_{n+m}(|\widehat{G}(X)|$, ch). Hence it is sufficient to show that $\operatorname{ch}_{n+m}\left(\widehat{m}_{*}^{G}(x, y)\right)=\operatorname{ch}_{n}(x) \bullet \operatorname{ch}_{m}(y)$.

For $x=[(f, \omega)]$ and $y=[(g, \tau)]$, Theorem 5.2 implies that

$$
\begin{aligned}
\operatorname{ch}_{n+m}\left(\widehat{m}_{*}^{G}(x, y)\right)= & \operatorname{ch}_{n+m}(f \times g)+d_{\mathcal{D}}\left((-1)^{n} \operatorname{ch}_{n}(f) \bullet \tau+\omega \bullet \operatorname{ch}_{m}(g)\right. \\
& \left.+\omega \bullet d_{\mathcal{D}} \tau+(-1)^{n} \operatorname{ch}_{n}(f) \triangle \operatorname{ch}_{m}(g)\right) \\
= & \left(\operatorname{ch}_{n}(f)+d_{\mathcal{D}} \omega\right) \bullet\left(\operatorname{ch}_{m}(g)+d_{\mathcal{D}} \tau\right) \\
= & \operatorname{ch}_{n}(x) \bullet \operatorname{ch}_{m}(y)
\end{aligned}
$$

The case where $n=0$ or $m=0$ is trivial.

Remark 1. The map Cub : $\widehat{S}_{n+1} \widehat{S}_{m+1}(X) \rightarrow \widehat{\mathrm{Cub}}_{n+m}(X)$ gives rise to a map

$$
\text { Cub : } C_{*}\left(\left|\widehat{S}^{(2)}(X)\right|\right)[-2] \rightarrow \widehat{\operatorname{Cub}}_{*}(X)
$$

and the tensor product of hermitian vector bundles induces a map

$$
C_{*}(|\widehat{S}(X)|)[-1] \otimes C_{*}(|\widehat{S}(X)|)[-1] \rightarrow C_{*}\left(\left|\widehat{S}^{(2)}(X)\right|\right)[-2] .
$$

But both of them are not compatible with the differentials. So it seems impossible to the author to define a product in $\widehat{K}_{*}(X)$ by using the $S$-construction.

Remark 2. In [6], another complex $\mathcal{H}_{T W}^{*}(X, p)$ computing real Deligne cohomology and higher Bott-Chern form with values in this complex are introduced. In the same argument as in $\S 4.1$, we can prove that this Bott-Chern form of any degenerate element of $\widehat{S}(X)$ is zero. Hence we have

$$
\operatorname{ch}_{T W}: C_{*}(|\widehat{S}(X)|) \rightarrow \underset{p}{\oplus} \mathcal{H}_{T W}^{2 p-*}(X, p),
$$

and we can define a new version of higher arithmetic $K$-theory:

$$
\widehat{K}_{n}^{T W}(X)=\widehat{\pi}_{n+1}\left(|\widehat{S}(X)|, \operatorname{ch}_{T W}\right) .
$$


The complex $\mathcal{H}_{T W}^{*}(X, p)$ is much bigger than $\mathcal{D}^{*}(X, p)$, therefore $\widehat{K}_{n}^{T W}(X)$ is not isomorphic to $\widehat{K}_{n}(X)$ even in the case of $n=0$.

The advantage of working with $\mathcal{H}_{T W}^{*}(X, p)$ rather than $\mathcal{D}^{*}(X, p)$ is the multiplicative property of $\operatorname{ch}_{T W}$. In fact, it is proved in $[6, \S 6]$ that $\mathcal{H}_{T W}^{*}(X, p)$ is equipped with graded commutative and associative product and $\mathrm{ch}_{T W}$ respects the product structures on the both sides. Hence in this case we do not need to deal with the operation $\triangle$ which compensates for the lack of compatibility of Bott-Chern forms with products, and we can define product in $\widehat{K}_{*}^{T W}(X)$ in a simpler form. Moreover, it can be proved that the product in $\widehat{K}_{*}^{T W}(X)$ satisfies the associative law.

\section{$\S 6.6$. The commutativity of the product}

In this subsection we discuss the commutativity of the product in $\widehat{K}_{*}(X)$. When $n=0$ or $m=0$, it is easy to prove that the product $\widehat{K}_{n}(X) \times \widehat{K}_{m}(X) \rightarrow$ $\widehat{K}_{n+m}(X)$ is commutative. So we concentrate to the case of $n, m \geq 1$.

For a small exact category $\mathfrak{A}$, let $L: G_{n} \mathfrak{A} \rightarrow G_{n} G_{0} \mathfrak{A}$ be the map given by $L\left(E^{+}, E^{-}\right)=\left(E^{+}, 0, E^{-}, 0\right)$. Then it induces a homotopy equivalence $L$ : $G \mathfrak{A} \rightarrow G^{(2)} \mathfrak{A}$ and it is homotopy equivalent to the map $R$. Similarly to the case of the map $R$, we can obtain an isomorphism

$$
\widehat{L}_{*}: \widehat{\pi}_{n}(|\widehat{G}(X)|, \mathrm{ch}) \simeq \widehat{\pi}_{n}\left(\left|\widehat{G}^{(2)}(X)\right|, \mathrm{ch}\right) .
$$

Definition 6.9. For $n, m \geq 1$, we define a new product

$$
\underset{L}{\times}: \widehat{K}_{n}(X) \times \widehat{K}_{m}(X) \rightarrow \widehat{K}_{n+m}(X)
$$

by

$$
\widehat{\pi}_{n}(|\widehat{G}(X)|, \mathrm{ch}) \times \widehat{\pi}_{m}(|\widehat{G}(X)|, \mathrm{ch}) \stackrel{\widehat{m}_{*}^{G}}{\rightarrow} \widehat{\pi}_{m}\left(\left|\widehat{G}^{(2)}(X)\right|, \mathrm{ch}\right) \stackrel{\widehat{L}_{*}^{-1}}{\rightarrow} \widehat{\pi}_{m}(|\widehat{G}(X)|, \mathrm{ch}) .
$$

Let us compare this new product with the one given in the previous section. Let $T: \widehat{S}_{n} \widehat{S}_{m}(X) \rightarrow \widehat{S}_{m} \widehat{S}_{n}(X)$ be the switching map $T(E)_{(i, j) \times(\alpha, \beta)}=$ $E_{(\alpha, \beta) \times(i, j)}$. Then the map

$$
\coprod_{n, m} \widehat{G}_{n} \widehat{G}_{m}(X) \times \Delta^{n} \times \Delta^{m} \rightarrow \coprod_{n, m} \widehat{G}_{m} \widehat{G}_{n}(X) \times \Delta^{m} \times \Delta^{n}
$$

given by $\left(E^{ \pm \pm}, t_{1}, t_{2}\right) \mapsto\left(T\left(E^{ \pm \pm}\right), t_{2}, t_{1}\right)$ induces an involution $\mathcal{T}$ on $\left|\widehat{G}^{(2)}(X)\right|$. 
Lemma 6.10. The diagram

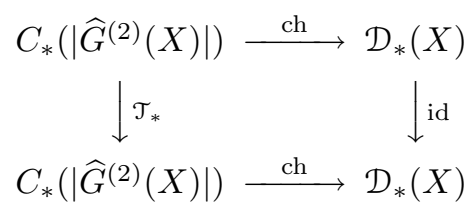

is commutative. Hence we can obtain an isomorphism

$$
\widehat{\mathcal{T}}_{*}: \widehat{\pi}_{n}\left(\left|\widehat{G}^{(2)}(X)\right|, \mathrm{ch}\right) \simeq \widehat{\pi}_{n}\left(\left|\widehat{G}^{(2)}(X)\right|, \mathrm{ch}\right)
$$

by $[(f, \omega)] \mapsto[(\mathcal{T} f, \omega)]$.

Proof. If we denote by $[E]$ the element of $C_{*}\left(\left|\widehat{G}^{(2)}(X)\right|\right)$ determined by $E \in \widehat{G}_{n} \widehat{G}_{m}(X)$, then $\mathcal{T}_{*}([E])=(-1)^{n m}[T(E)]$. Hence we have

$$
\begin{aligned}
\operatorname{ch}_{n+m}\left(\mathcal{T}_{*}([E])\right) & =(-1)^{n m} \operatorname{ch}_{n+m}(\operatorname{Cub}(T(E))) \\
& =(-1)^{n m} \operatorname{ch}_{n+m}\left(T_{n, m}(\operatorname{Cub}(E))\right),
\end{aligned}
$$

where $T_{n, m}(\mathcal{F})$ for an exact hermitian $(n+m)$-cube $\mathcal{F}$ is given by $T_{n, m}$ $(\mathcal{F})_{\alpha_{1}, \ldots, \alpha_{n+m}}=\mathcal{F}_{\alpha_{n+1}, \ldots, \alpha_{n+m}, \alpha_{1}, \ldots, \alpha_{n}}$. Then it is easy to see that $\operatorname{ch}_{n+m}$ $\left(T_{n, m}(\mathcal{F})\right)=(-1)^{n m} \operatorname{ch}_{n+m}(\mathcal{F})$. Hence we can say that $\operatorname{ch}_{n+m}\left(\mathcal{T}_{*}([E])\right)=$ $\operatorname{ch}_{n+m}(E)$.

Proposition 6.11. Let $x \in \widehat{K}_{n}(X)$ and $y \in \widehat{K}_{m}(X)$ with $n, m \geq 1$. Then we have

$$
x \times y=(-1)^{n m} y \underset{L}{\times} x .
$$

Proof. For two pointed CW-complexes $S_{1}$ and $S_{2}$, let $T: S_{1} \wedge S_{2} \rightarrow S_{2} \wedge S_{1}$ denote the map given by $T\left(s_{1}, s_{2}\right)=\left(s_{2}, s_{1}\right)$. For two pointed cellular maps $f: S^{n} \rightarrow|\widehat{G}(X)|$ and $g: S^{m} \rightarrow|\widehat{G}(X)|$, we consider the following diagram:

$$
\begin{aligned}
& S^{n} \wedge S^{m} \stackrel{f \wedge g}{\longrightarrow}|\widehat{G}(X)| \wedge|\widehat{G}(X)| \stackrel{m^{G}}{\longrightarrow}\left|\widehat{G}^{(2)}(X)\right| \\
& \downarrow T \quad \downarrow T \quad \downarrow \mathcal{T} \\
& S^{m} \wedge S^{n} \stackrel{g \wedge f}{\longrightarrow}|\widehat{G}(X)| \wedge|\widehat{G}(X)| \stackrel{m^{G}}{\longrightarrow}\left|\widehat{G}^{(2)}(X)\right| .
\end{aligned}
$$

The left square is obviously commutative, but the right one is not. In fact, for $E \in \widehat{G}_{n}(X)$ and $F \in \widehat{G}_{m}(X)$ we have $\mathcal{T} m^{G}(E, F)_{(i, j) \times(\alpha, \beta)}=E_{\alpha, \beta} \otimes F_{i, j}$ and $m^{G} T(E, F)_{(i, j) \times(\alpha, \beta)}=F_{i, j} \otimes E_{\alpha, \beta}$. Hence a homotopy from $\mathcal{T} m^{G}$ to $m^{G} T$ is given by means of the canonical isometry $\bar{P} \otimes \bar{Q} \simeq \bar{Q} \otimes \bar{P}$. Hence we can show that $[(\mathcal{T}(f \times g), 0)]=[((g \times f) T, 0)]$ in the same way as the proof of Lemma 6.5. 
If $x=[(f, \omega)]$ and $y=[(g, \tau)]$, then we have

$$
\begin{aligned}
\widehat{\mathcal{T}}_{*} \widehat{m}_{*}^{G} & ([(f, \omega)],[(g, \tau)]) \\
=[ & {\left[\left(\mathcal{T}(f \times g),(-1)^{n} \operatorname{ch}_{n}(f) \bullet \tau+\omega \bullet \operatorname{ch}_{m}(g)+\omega \bullet d_{\mathcal{D}} \tau\right.\right.} \\
& \left.\left.+(-1)^{n} \operatorname{ch}_{n}(f) \triangle \operatorname{ch}_{m}(g)\right)\right] \\
= & {\left[(g \times f) T,(-1)^{n m} \tau \bullet \operatorname{ch}_{n}(f)+(-1)^{(n+1) m} \operatorname{ch}_{m}(g) \bullet \omega\right.} \\
& \left.\left.+(-1)^{n m} \tau \bullet d_{\mathcal{D}} \omega+(-1)^{(n+1) m} \operatorname{ch}_{m}(g) \Delta \operatorname{ch}_{n}(f)\right)\right] .
\end{aligned}
$$

Since $T: S^{n+m} \rightarrow S^{n+m}$ is homotopic to $(-1)^{n m} \operatorname{id}_{S^{n+m}}$, we have

$$
\widehat{\mathcal{T}}_{*} \widehat{m}_{*}^{G}([(f, \omega)],[(g, \tau)])=(-1)^{n m} \widehat{m}_{*}^{G}([(g, \tau)],[(f, \omega)])
$$

in $\widehat{\pi}_{n+m}\left(\left|\widehat{G}^{(2)}(X)\right|\right.$, ch). Hence

$$
\begin{aligned}
(-1)^{n m} \widehat{L}_{*}([(g, \tau)] \underset{L}{\times[}[(f, \omega)]) & =(-1)^{n m} \widehat{m}_{*}^{G}([(g, \tau)],[(f, \omega)]) \\
& =\widehat{\mathcal{T}}_{*} \widehat{m}_{*}^{G}([(f, \omega)],[(g, \tau)]) \\
& =\widehat{\mathcal{T}}_{*} \widehat{R}_{*}([(f, \omega)] \times[(g, \tau)]) \\
& =\widehat{L}_{*}([(f, \omega)] \times[(g, \tau)]) .
\end{aligned}
$$

Since $\widehat{L}_{*}$ is bijective, we have completed the proof.

Proposition 6.12. For $x \in \widehat{\pi}_{n}(|\widehat{G}(X)|, \mathrm{ch}), \widehat{R}_{*}(x)-\widehat{L}_{*}(x)$ is contained in $\operatorname{Im}\left(\widetilde{\mathcal{D}}_{n+1}(X) \rightarrow \widehat{\pi}_{n}\left(\left|\widehat{G}^{(2)}(X)\right|\right.\right.$, ch $\left.)\right)$ and $2\left(\widehat{R}_{*}(x)-\widehat{L}_{*}(x)\right)=0$. In particular, for $x \in \widehat{K}_{n}(X)$ and $y \in \widehat{K}_{m}(X)$ with $n, m \geq 1, x \times y-\underset{L}{\times} y$ is contained in $\operatorname{Im}\left(\widetilde{\mathcal{D}}_{n+m+1}(X) \rightarrow \widehat{K}_{n+m}(X)\right)$ and $2(x \times y-\underset{L}{\times} y)=0$.

Proof. Since $R$ is homotopy equivalent to $L, \widehat{R}_{*}(x)-\widehat{L}_{*}(x)$ is contained in $\operatorname{Im}\left(\widetilde{\mathcal{D}}_{n+1}(X) \rightarrow \widehat{\pi}_{n}\left(\left|\widehat{G}^{(2)}(X)\right|\right.\right.$, ch $\left.)\right)$. If $f: S^{n} \rightarrow|\widehat{G}(X)|$ is a pointed cellular map, then there is a pointed cellular map

$$
H:\left(S^{n} \times I\right) /(\{*\} \times I) \rightarrow\left|\widehat{G}^{(2)}(X)\right|
$$

such that $H(s, 0)=R f(s)$ and $H(s, 1)=L f(s)$. Let

$$
H^{\prime}=\mathcal{T} H:\left(S^{n} \times I\right) /(\{*\} \times I) \rightarrow\left|\widehat{G}^{(2)}(X)\right|,
$$

then we have $H^{\prime}(s, 0)=L f(s)$ and $H^{\prime}(s, 1)=R f(s)$. The commutative square in Lemma 6.10 implies that $\operatorname{ch}_{n+1}\left(H^{\prime}\right)=\operatorname{ch}_{n+1}(H)$. Gluing the maps $H$ and $H^{\prime}$ on the boundaries, we obtain a cellular map

$$
H \cup H^{\prime}:\left(S^{n} \times T^{1}\right) /\left(\{*\} \times T^{1}\right) \rightarrow\left|\widehat{G}^{(2)}(X)\right|,
$$

where $T^{1}$ is the barycentric subdivision of $S^{1}$. 
Lemma 6.13. If $n \geq 1$, there is a surjection

$$
p: S^{n+1} \rightarrow\left(S^{n} \times S^{1}\right) /\left(\{*\} \times S^{1}\right)
$$

such that $p^{-1}\left(\left(S^{n}-\{*\}\right) \times S^{1}\right) \rightarrow\left(S^{n}-\{*\}\right) \times S^{1}$ is a homeomorphism.

Proof. We describe the space $S^{n+1}$ as follows:

$$
S^{n+1}=\left\{\left(z, t_{1}, \ldots, t_{n}\right) \in \mathbb{C} \times \mathbb{R}^{n} ;|z|^{2}+t_{1}^{2}+\cdots+t_{n}^{2}=1\right\} .
$$

Let $S^{n-1}=\left\{\left(0, t_{1}, \ldots, t_{n}\right) \in S^{n+1}\right\}$. Then the map $S^{n+1} \backslash S^{n-1} \rightarrow B^{n} \times S^{1}$ given by

$$
\left(z, t_{1}, \ldots, t_{n}\right) \mapsto\left(\left(t_{1}, \ldots, t_{n}\right), \frac{z}{\sqrt{1-t_{1}^{2}-\cdots-t_{n}^{2}}}\right)
$$

is a homeomorphism, where $B^{n}=\left\{\left(t_{1}, \ldots, t_{n}\right) \in \mathbb{R}^{n} ; t_{1}^{2}+\cdots+t_{n}^{2}<1\right\}$. Since $\left(S^{n} \times S^{1}\right) /\left(\{*\} \times S^{1}\right)$ is the one-point compactification of $B^{n} \times S^{1}$, this homeomorphism can be extended to the map $p: S^{n+1} \rightarrow\left(S^{n} \times S^{1}\right) /\left(\{*\} \times S^{1}\right)$ which satisfies the above condition.

Let us return to the proof of Proposition 6.12. Since $T^{1}$ is the barycentric subdivision of $S^{1}$, the Bott-Chern form of the map

$$
F: S^{n+1} \stackrel{p}{\longrightarrow}\left(S^{n} \times T^{1}\right) /\left(\{*\} \times T^{1}\right) \stackrel{H \cup H^{\prime}}{\longrightarrow}\left|\widehat{G}^{(2)}(X)\right|
$$

is $2 \mathrm{ch}_{n+1}(H)$ up to sign. Therefore $2 \mathrm{ch}_{n+1}(H)$ is contained in the image of $\pi_{n+1}\left(\left|\widehat{G}^{(2)}(X)\right|\right) \rightarrow \widetilde{\mathcal{D}}_{n+1}(X)$. Hence $2\left[\left(0, \mathrm{ch}_{n+1}(H)\right)\right]=0$ in $\widehat{\pi}_{n}\left(\left|\widehat{G}^{(2)}(X)\right|, \mathrm{ch}\right)$ by Theorem 3.3. For $x=[(f, \omega)] \in \widehat{\pi}_{n}(|\widehat{G}(X)|$, ch),

$$
\begin{aligned}
\widehat{R}_{*}(x)-\widehat{L}_{*}(x) & =[(R f, 0)]-[(L f, 0)] \\
& =(-1)^{n+1}\left[\left(0, \operatorname{ch}_{n+1}(H)\right)\right],
\end{aligned}
$$

therefore $2\left(\widehat{R}_{*}(x)-\widehat{L}_{*}(x)\right)=0$.

Combining Proposition 6.11 with Proposition 6.12 yields the following:

Theorem 6.14. Let $x \in \widehat{K}_{n}(X)$ and $y \in \widehat{K}_{m}(X)$. Then $x \times y-$ $(-1)^{n m} y \times x$ is a 2-torsion element contained in $\operatorname{Im}\left(\widetilde{\mathcal{D}}_{n+m+1}(X) \rightarrow \widehat{K}_{n+m}(X)\right)$. Hence the product in $\widehat{K}_{*}(X)$ is graded commutative up to 2-torsion. 


\section{$\S 6.7$. The lack of the associativity}

In this subsection we discuss the associativity of the product in $\widehat{K}_{*}(X)$. Let $\widehat{G}^{(3)}(X)$ be the trisimplicial set given by taking $\widehat{G}$ three times. Then the tensor product of hermitian vector bundles gives the following maps:

$$
\begin{aligned}
& m^{G}: \widehat{G}^{(2)}(X) \wedge \widehat{G}(X) \rightarrow \widehat{G}^{(3)}(X), \\
& m^{G}: \widehat{G}(X) \wedge \widehat{G}^{(2)}(X) \rightarrow \widehat{G}^{(3)}(X) .
\end{aligned}
$$

Let $R: \widehat{G}(X) \rightarrow \widehat{G}^{(3)}(X)$ be a homotopy equivalent map given by $R(E)^{++ \pm}=$ $E^{ \pm}$and $R(E)^{+- \pm}=R(E)^{-+ \pm}=R(E)^{-- \pm}=0$ for $E=\left(E^{+}, E^{-}\right) \in \widehat{G}_{n}(X)$. Under the above notations, the following diagram

$$
\begin{array}{ccc}
\widehat{G}(X) \wedge \widehat{G}(X) \wedge \widehat{G}(X) \stackrel{m^{G} \wedge 1}{\longrightarrow} & \widehat{G}^{(2)}(X) \wedge \widehat{G}(X) \\
\downarrow 1 \wedge m^{G} & & \downarrow m^{G} \\
\widehat{G}(X) \wedge \widehat{G}^{(2)}(X) & \stackrel{m^{G}}{\longrightarrow} & \widehat{G}^{(3)}(X)
\end{array}
$$

is commutative up to a homotopy arising from the natural isometry $(\bar{E} \otimes \bar{F}) \otimes$ $\bar{G} \simeq \bar{E} \otimes(\bar{F} \otimes \bar{G})$. This commutative diagram implies the associativity of the product in usual algebraic $K$-theory $K_{*}(X)$.

For two pointed cellular maps $f: S^{n} \rightarrow\left|\widehat{G}^{(2)}(X)\right|$ and $g: S^{m} \rightarrow|\widehat{G}(X)|$, let

$$
f \times g: S^{n+m} \stackrel{f \wedge g}{\longrightarrow}\left|\widehat{G}^{(2)}(X)\right| \wedge|\widehat{G}(X)| \stackrel{\widehat{m}^{G}}{\longrightarrow}\left|\widehat{G}^{(3)}(X)\right| .
$$

We define a pairing

$$
\widehat{m}_{*}^{G}: \widehat{\pi}_{n}\left(\left|\widehat{G}^{(2)}(X)\right|, \mathrm{ch}\right) \times \widehat{\pi}_{m}(|\widehat{G}(X)|, \mathrm{ch}) \rightarrow \widehat{\pi}_{n+m}\left(\left|\widehat{G}^{(3)}(X)\right|, \mathrm{ch}\right)
$$

by

$$
\begin{aligned}
& ([(f, \omega)],[(g, \tau)]) \mapsto \\
& \quad\left[\left(f \times g,(-1)^{n} \operatorname{ch}_{n}(f) \bullet \tau+\omega \bullet \operatorname{ch}_{m}(g)+\omega \bullet d_{\mathcal{D}} \tau+(-1)^{n} \operatorname{ch}_{n}(f) \Delta \operatorname{ch}_{m}(g)\right)\right] .
\end{aligned}
$$

The well-definedness of the pairing can be verified in the same way as the proof of Proposition 6.6. We can also define a pairing

$$
\widehat{m}_{*}^{G}: \widehat{\pi}_{n}(|\widehat{G}(X)|, \mathrm{ch}) \times \widehat{\pi}_{m}\left(\left|\widehat{G}^{(2)}(X)\right|, \mathrm{ch}\right) \rightarrow \widehat{\pi}_{n+m}\left(\left|\widehat{G}^{(3)}(X)\right|, \mathrm{ch}\right)
$$

by the same expression as above. Then the associativity of the product in 
$\widehat{K}_{*}(X)$ is equivalent to the commutativity of the following diagram:

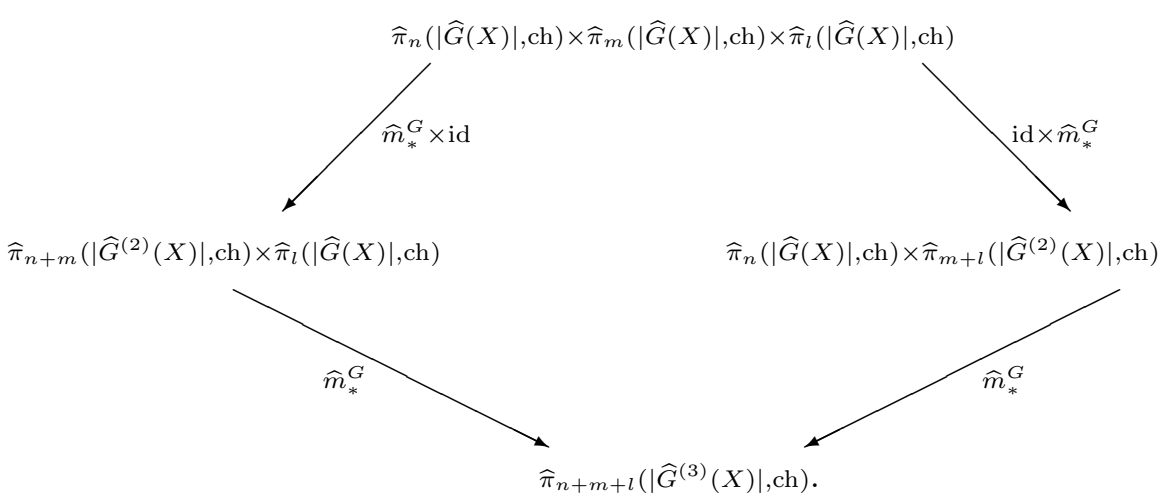

However, the diagram is not commutative. Take $[(f, \omega)] \in \widehat{\pi}_{n}(|\widehat{G}(X)|, \mathrm{ch})$, $[(g, \tau)] \in \widehat{\pi}_{m}(|\widehat{G}(X)|, \mathrm{ch})$ and $[(h, \eta)] \in \widehat{\pi}_{l}(|\widehat{G}(X)|, \mathrm{ch})$. Then in the same way as the proof of Lemma 6.5 , we can prove the identity

$$
[((f \times g) \times h, 0)]=[(f \times(g \times h), 0)]
$$

in $\widehat{\pi}_{n+m+l}\left(\left|\widehat{G}^{(3)}(X)\right|, \mathrm{ch}\right)$. Hence an easy calculation implies the following:

Proposition 6.15. We have

$$
\begin{aligned}
\widehat{m}_{*}^{G} & \left(\widehat{m}_{*}^{G}([(f, \omega)],[(g, \tau)]),[(h, \eta)]\right)-\widehat{m}_{*}^{G}\left([(f, \omega)], \widehat{m}_{*}^{G}([(g, \tau)],[(h, \eta)])\right) \\
& =[(0, r(f, g, h, \omega, \tau, \eta))]
\end{aligned}
$$

in $\widehat{\pi}_{n+m+l}\left(\left|\widehat{G}^{(3)}(X)\right|, \mathrm{ch}\right)$, where

$$
\begin{aligned}
r(f, g, h, \omega, \tau, \eta) \\
=(-1)^{n}\left(\left(\operatorname{ch}_{n}(f)+d_{\mathcal{D}} \omega\right) \bullet \tau\right) \bullet\left(\operatorname{ch}_{l}(h)+d_{\mathcal{D}} \eta\right) \\
\quad-(-1)^{n}\left(\operatorname{ch}_{n}(f)+d_{\mathcal{D}} \omega\right) \bullet\left(\tau \bullet\left(\operatorname{ch}_{l}(h)+d_{\mathcal{D}} \eta\right)\right) \\
\quad+(-1)^{n+m} \operatorname{ch}_{n+m}(f \times g) \bullet \eta+(-1)^{n}\left(\operatorname{ch}_{n}(f) \Delta \operatorname{ch}_{m}(g)\right) \bullet d_{\mathcal{D}} \eta \\
\quad-(-1)^{n+m} \operatorname{ch}_{n}(f) \bullet\left(\operatorname{ch}_{m}(g) \bullet \eta\right) \\
\quad+\left(\omega \bullet \operatorname{ch}_{m}(g)\right) \bullet \operatorname{ch}_{l}(h)-(-1)^{n+m} d_{\mathcal{D}} \omega \bullet\left(\operatorname{ch}_{m}(g) \Delta \operatorname{ch}_{l}(h)\right) \\
\quad-\omega \bullet \operatorname{ch}_{m+l}(g \times h)+\left(\omega \bullet \operatorname{ch}_{m}(g)\right) \bullet d_{\mathcal{D}} \eta-(-1)^{n+m} d_{\mathcal{D}} \omega \bullet\left(\operatorname{ch}_{m}(g) \bullet \eta\right) \\
\quad+(-1)^{n}\left(\operatorname{ch}_{n}(f) \Delta \operatorname{ch}_{m}(g)\right) \bullet \operatorname{ch}_{l}(h)+(-1)^{n+m} \operatorname{ch}_{n+m}(f \times g) \Delta \operatorname{ch}_{l}(h) \\
\quad-(-1)^{n+m} \operatorname{ch}_{n}(f) \bullet\left(\operatorname{ch}_{m}(g) \Delta \operatorname{ch}_{l}(h)\right)-(-1)^{n} \operatorname{ch}_{n}(f) \Delta \operatorname{ch}_{m+l}(g \times h) .
\end{aligned}
$$


Lemma 6.16. Assume $n m l \geq 1$ and let $\alpha \in \mathcal{D}_{n}(X), \beta \in \mathcal{D}_{m}(X)$ and $\gamma \in \mathcal{D}_{l}(X)$.

1) We have

$$
\begin{aligned}
& (\alpha \bullet \beta) \bullet \gamma-\alpha \bullet(\beta \bullet \gamma) \\
& =(-1)^{n+m}\left(\partial \alpha^{(-1,-n)}+\bar{\partial} \alpha^{(-n,-1)}\right) \wedge\left(\partial \beta^{(-1,-m)}+\bar{\partial} \beta^{(-m,-1)}\right) \wedge \gamma \\
& \quad-\alpha \wedge\left(\partial \beta^{(-1,-m)}+\bar{\partial} \beta^{(-m,-1)}\right) \wedge\left(\partial \gamma^{(-1,-l)}+\bar{\partial} \gamma^{(-l,-1)}\right) .
\end{aligned}
$$

2) If $d_{\mathcal{D}} \alpha=d_{\mathcal{D}} \beta=0$, then

$$
(\alpha \bullet \beta) \bullet \gamma-\alpha \bullet(\beta \bullet \gamma) \equiv \begin{cases}-\alpha \wedge d \beta \wedge d_{\mathcal{D}} \gamma, & l \geq 2 \\ 0, & l=1\end{cases}
$$

modulo $\operatorname{Im} d_{\mathcal{D}}$.

3) If $d_{\mathcal{D}} \beta=d_{\mathcal{D}} \gamma=0$, then

$$
(\alpha \bullet \beta) \bullet \gamma-\alpha \bullet(\beta \bullet \gamma) \equiv \begin{cases}(-1)^{n+m} d_{\mathcal{D}} \alpha \wedge d \beta \wedge \gamma, & n \geq 2 \\ 0, & n=1\end{cases}
$$

modulo $\operatorname{Im} d_{\mathcal{D}}$.

4) If $d_{\mathcal{D}} \alpha=d_{\mathcal{D}} \gamma=0$, then

$$
(\alpha \bullet \beta) \bullet \gamma-\alpha \bullet(\beta \bullet \gamma) \equiv \begin{cases}(-1)^{m} \alpha \wedge d d_{\mathcal{D}} \beta \wedge \gamma, & m \geq 2 \\ 0, & m=1\end{cases}
$$

$\operatorname{modulo} \operatorname{Im} d_{\mathcal{D}}$.

Proof. The identity in 1) follows from an easy calculation. If $l \geq 2$ and $d_{\mathcal{D}} \alpha=d_{\mathcal{D}} \beta=0$, then

$$
\begin{aligned}
& (\alpha \bullet \beta) \bullet \gamma-\alpha \bullet(\beta \bullet \gamma) \\
& \quad=(-1)^{n+m} d \alpha \wedge d \beta \wedge \gamma-\alpha \wedge d \beta \wedge\left(d \gamma+d_{\mathcal{D}} \gamma\right) \\
& \quad=(-1)^{n+m} d(\alpha \wedge d \beta \wedge \gamma)-\alpha \wedge d \beta \wedge d_{\mathcal{D}} \gamma .
\end{aligned}
$$

The form $\alpha \wedge d \beta \wedge \gamma$ is contained in $\mathcal{D}^{2(p+q+r)-n-m-l-1}(X, p+q+r)$ and $d_{\mathcal{D}}(\alpha \wedge d \beta \wedge \gamma)=-d(\alpha \wedge d \beta \wedge \gamma)$. Hence we have

$$
(\alpha \bullet \beta) \bullet \gamma-\alpha \bullet(\beta \bullet \gamma) \equiv-\alpha \wedge d \beta \wedge d_{\mathcal{D}} \gamma
$$

modulo $\operatorname{Im} d_{\mathcal{D}}$. When $l=1$, we have

$$
(\alpha \bullet \beta) \bullet \gamma-\alpha \bullet(\beta \bullet \gamma)=(-1)^{n+m+1} d_{\mathcal{D}}(\alpha \wedge d \beta \wedge \gamma)
$$


Hence 2) holds. The identities in 3) and 4) can be proved in the same way.

Let us calculate $r(f, g, h, \omega, \tau, \eta)$ by using Lemma 6.16. If $n m l \geq 1$, then

$$
\begin{aligned}
& (-1)^{n}\left(\left(\operatorname{ch}_{n}(f)+d_{\mathcal{D}} \omega\right) \bullet \tau\right) \bullet\left(\operatorname{ch}_{l}(h)+d_{\mathcal{D}} \eta\right) \\
& -(-1)^{n}\left(\operatorname{ch}_{n}(f)+d_{\mathcal{D}} \omega\right) \bullet\left(\tau \bullet\left(\operatorname{ch}_{l}(h)+d_{\mathcal{D}} \eta\right)\right) \\
& \quad \equiv(-1)^{n+m+1}\left(\operatorname{ch}_{n}(f)+d_{\mathcal{D}} \omega\right) \wedge d d_{\mathcal{D}} \tau \wedge\left(\operatorname{ch}_{l}(h)+d_{\mathcal{D}} \eta\right)
\end{aligned}
$$

and

$$
\begin{aligned}
& \left(\omega \bullet \operatorname{ch}_{m}(g)\right) \bullet d_{\mathcal{D}} \eta-(-1)^{n+m} d_{\mathcal{D}} \omega \bullet\left(\operatorname{ch}_{m}(g) \bullet \eta\right) \\
& \quad \equiv(-1)^{n+m+1} d_{\mathcal{D}} \omega \wedge d \operatorname{ch}_{m}(g) \wedge d_{\mathcal{D}} \eta
\end{aligned}
$$

modulo $\operatorname{Im} d_{\mathcal{D}}$. Since

$$
\operatorname{ch}_{n+m}(f \times g)=\operatorname{ch}_{n}(f) \bullet \operatorname{ch}_{m}(g)+(-1)^{n+1} d_{\mathcal{D}}\left(\operatorname{ch}_{n}(f) \triangle \operatorname{ch}_{m}(g)\right)
$$

by Theorem 5.2 , we have

$$
\begin{aligned}
(-1)^{n+m} & \operatorname{ch}_{n+m}(f \times g) \bullet \eta+(-1)^{n}\left(\operatorname{ch}_{n}(f) \Delta \operatorname{ch}_{m}(g)\right) \bullet d_{\mathcal{D}} \eta \\
& -(-1)^{n+m} \operatorname{ch}_{n}(f) \bullet\left(\operatorname{ch}_{m}(g) \bullet \eta\right) \\
\equiv & (-1)^{n+m}\left(\operatorname{ch}_{n}(f) \bullet \operatorname{ch}_{m}(g)\right) \bullet \eta-(-1)^{n+m} \operatorname{ch}_{n}(f) \bullet\left(\operatorname{ch}_{m}(g) \bullet \eta\right) \\
\equiv & (-1)^{n+m+1} \operatorname{ch}_{n}(f) \wedge d \operatorname{ch}_{m}(g) \wedge d_{\mathcal{D}} \eta
\end{aligned}
$$

modulo $\operatorname{Im} d_{\mathcal{D}}$. In the same way we have

$$
\begin{aligned}
& \left(\omega \bullet \operatorname{ch}_{m}(g)\right) \bullet \operatorname{ch}_{l}(h)-\omega \bullet \operatorname{ch}_{m+l}(g \times h)-(-1)^{n+m} d_{\mathcal{D}} \omega \bullet\left(\operatorname{ch}_{m}(g) \Delta \operatorname{ch}_{l}(h)\right) \\
& \quad \equiv(-1)^{n+m+1} d_{\mathcal{D}} \omega \wedge d \operatorname{ch}_{m}(g) \wedge \operatorname{ch}_{l}(h)
\end{aligned}
$$

modulo $\operatorname{Im} d_{\mathcal{D}}$. As for the last four terms, we have the following:

Proposition 6.17. If $n m l \geq 1$, then we have

$$
\begin{array}{r}
(-1)^{n}\left(\operatorname{ch}_{n}(f) \Delta \operatorname{ch}_{m}(g)\right) \bullet \operatorname{ch}_{l}(h)-(-1)^{n+m} \operatorname{ch}_{n}(f) \bullet\left(\operatorname{ch}_{m}(g) \Delta \operatorname{ch}_{l}(h)\right) \\
+(-1)^{n+m} \operatorname{ch}_{n+m}(f \times g) \Delta \operatorname{ch}_{l}(h)-(-1)^{n} \operatorname{ch}_{n}(f) \Delta \operatorname{ch}_{m+l}(g \times h) \\
\equiv(-1)^{n+m+1} \operatorname{ch}_{n}(f) \wedge d \operatorname{ch}_{m}(g) \wedge \operatorname{ch}_{l}(h)
\end{array}
$$

modulo $\operatorname{Im} d_{\mathcal{D}}$.

We will prove this proposition in $\S 6.9$. Substituting these identities into that in Proposition 6.15 yields that

$r(f, g, h, \omega, \tau, \eta) \equiv(-1)^{n+m+1}\left(\operatorname{ch}_{n}(f)+d_{\mathcal{D}} \omega\right) \wedge d\left(\operatorname{ch}_{m}(g)+d_{\mathcal{D}} \tau\right) \wedge\left(\operatorname{ch}_{l}(h)+d_{\mathcal{D}} \eta\right)$ $\operatorname{modulo} \operatorname{Im} d_{\mathcal{D}}$. 
Theorem 6.18. $\quad$ The product in higher arithmetic K-theory does not satisfy the associative law. In fact, if $x \in \widehat{K}_{n}(X), y \in \widehat{K}_{m}(X)$ and $z \in \widehat{K}_{l}(X)$ for $n m l \geq 1$, we have

$$
(x \times y) \times z-x \times(y \times z)=\left[\left(0,(-1)^{n+m+1} \operatorname{ch}_{n}(x) \wedge d \operatorname{ch}_{m}(y) \wedge \operatorname{ch}_{l}(z)\right)\right]
$$

in $\widehat{K}_{n+m+l}(X)$. Hence $(x \times y) \times z=x \times(y \times z)$ holds when $n m l=0$ or $y \in K_{m}(\bar{X})$ or $x=y=z$.

Proof. When $n m l \geq 1$, we have already proved this identity. The identity $(x \times y) \times z=x \times(y \times z)$ in the case of $n m l=0$ follows from the definition of the product and Lemma 6.16 .

\section{§6.8. Product in Arakelov $K$-theory}

For a proper Arakelov variety $\bar{X}=\left(X, h_{X}\right)$, let us define a pairing

$$
K_{n}(\bar{X}) \times K_{m}(\bar{X}) \rightarrow K_{n+m}(\bar{X})
$$

by $(x, y) \mapsto \sigma(x \times y)$, where $\sigma$ is the harmonic projection defined in $\S 4.3$.

Theorem 6.19. The above pairing makes $K_{*}(\bar{X})$ a graded associative algebra. That is to say, it follows that

$$
\sigma(\sigma(x \times y) \times z)=\sigma(x \times \sigma(y \times z))
$$

for $x, y, z \in K_{*}(\bar{X})$.

Proof. This identity is obvious when $n m l=0$, so we may assume that $n m l \geq 1$. We first prove the identity

$$
\sigma(\sigma(x \times y) \times z)=\sigma((x \times y) \times z)
$$

for $x \in K_{n}(\bar{X}), y \in K_{m}(\bar{X})$ and $z \in K_{l}(\bar{X})$. It follows from the definition of $\sigma$ that $\sigma(x \times y)=x \times y+[(0, \alpha)]$ where $\alpha \in \mathcal{D}_{n+m+1}(X)$ with $\mathcal{H}(\alpha)=0$. Then we have

$$
\sigma(x \times y) \times z=(x \times y) \times z+\left[\left(0, \alpha \bullet \operatorname{ch}_{l}(z)\right)\right],
$$

therefore

$$
\sigma(\sigma(x \times y) \times z)=(x \times y) \times z+\left[\left(0, \alpha \bullet \operatorname{ch}_{l}(z)+\beta\right)\right]
$$


where $\beta \in \mathcal{D}_{n+m+l+1}(X)$ with $\mathcal{H}(\beta)=0$. Let $\alpha^{\prime}$ be the sum of $(p-1, p-n-m)$ part of $\alpha$ and $\alpha^{\prime \prime}$ the sum of $(p-n-m, p-1)$-part of $\alpha$. Since $\operatorname{ch}_{l}(z)$ is harmonic,

$$
\alpha \bullet \operatorname{ch}_{l}(z)=(-1)^{n+m+1}\left(\partial \alpha^{\prime}-\bar{\partial} \alpha^{\prime \prime}\right) \wedge \operatorname{ch}_{l}(z) .
$$

Since $\partial \alpha^{\prime} \wedge \operatorname{ch}_{l}(z)$ is $\partial$-exact and $\bar{\partial} \alpha^{\prime \prime} \wedge \operatorname{ch}_{l}(z)$ is $\bar{\partial}$-exact, we have $\mathcal{H}\left(\alpha \bullet \operatorname{ch}_{l}(z)\right)=$ 0 , so $\mathcal{H}\left(\alpha \bullet \operatorname{ch}_{l}(z)+\beta\right)=0$. Therefore $\sigma(\sigma(x \times y) \times z)=\sigma((x \times y) \times z)$. In the same way, we can show that $\sigma(x \times \sigma(y \times z))=\sigma(x \times(y \times z))$. Hence by Thm. 6.18 we can obtain the desired identity.

\section{§6.9. Proof of Proposition 6.17}

For $\omega \in \mathcal{D}_{n}(X)$ and for an integer $i$ with $1 \leq i \leq n$, set

$$
\omega^{(-i,-n+i-1)}=\sum_{p} \omega^{(p-i, p-n+i-1)},
$$

where $\omega^{(p-i, p-n+i-1)}$ is the $(p-i, p-n+i-1)$-part of $\omega$. Then for $\omega \in \mathcal{D}_{n}(X)$ and $\tau \in \mathcal{D}_{m}(X)$, we can write $\omega \Delta \tau$ as follows:

$$
\omega \Delta \tau=\sum a_{i, j}^{n, m} \omega^{(-i,-n+i-1)} \wedge \tau^{(-j,-m+j-1)} .
$$

Set

$$
\begin{aligned}
\Phi= & (-1)^{n}\left(\operatorname{ch}_{n}(f) \Delta \operatorname{ch}_{m}(g)\right) \bullet \operatorname{ch}_{l}(h)-(-1)^{n+m} \operatorname{ch}_{n}(f) \bullet\left(\operatorname{ch}_{m}(g) \Delta \operatorname{ch}_{l}(h)\right) \\
& +(-1)^{n+m} \operatorname{ch}_{n+m}(f \times g) \Delta \operatorname{ch}_{l}(h)-(-1)^{n} \operatorname{ch}_{n}(f) \Delta \operatorname{ch}_{m+l}(g \times h),
\end{aligned}
$$

and let $\Phi(f)$ (resp. $\Phi(g)$ and $\Phi(h)$ ) be the part of $\Phi$ including the derivatives of $\operatorname{ch}_{n}(f)\left(\operatorname{resp} . \operatorname{ch}_{m}(g)\right.$ and $\left.\operatorname{ch}_{l}(h)\right)$. In other words, $\Phi=\Phi(f)+\Phi(g)+\Phi(h)$ such that

$$
\begin{gathered}
\Phi(f)=(-1)^{m+1}\left(\partial \operatorname{ch}_{n}(f)^{(-1,-n)}-\bar{\partial} \operatorname{ch}_{n}(f)^{(-n,-1)}\right) \wedge\left(\operatorname{ch}_{m}(g) \Delta \operatorname{ch}_{l}(h)\right) \\
+(-1)^{m}\left(\left(\partial \operatorname{ch}_{n}(f)^{(-1,-n)}-\bar{\partial} \operatorname{ch}_{n}(f)^{(-n,-1)}\right) \wedge \operatorname{ch}_{m}(g)\right) \Delta \operatorname{ch}_{l}(h) \\
+(-1)^{m}\left(\sum_{\substack{1 \leq i \leq n \\
1 \leq j \leq m}} a_{i, j}^{n, m} d \operatorname{ch}_{n}(f)^{(-n+i-1,-i)} \wedge \operatorname{ch}_{m}(g)^{(-m+j-1,-j)}\right) \Delta \operatorname{ch}_{l}(h), \\
\Phi(g)=(-1)^{n+m}\left(\operatorname{ch}_{n}(f) \wedge\left(\partial \operatorname{ch}_{m}(g)^{(-1,-m)}-\bar{\partial} \operatorname{ch}_{m}(g)^{(-m,-1)}\right)\right) \Delta \operatorname{ch}_{l}(h) \\
+(-1)^{n+m+1}\left(\sum_{\substack{1 \leq i \leq n \\
1 \leq j \leq m}} a_{i, j}^{n, m} \operatorname{ch}_{n}(f)^{(-n+i-1,-i)} \wedge d \operatorname{ch}_{m}(g)^{(-m+j-1,-j)}\right)
\end{gathered}
$$




$$
\begin{aligned}
& \Delta \operatorname{ch}_{l}(h) \\
+ & (-1)^{n+m+1} \operatorname{ch}_{n}(f) \Delta\left(\left(\partial \operatorname{ch}_{m}(g)^{(-1,-m)}-\bar{\partial} \operatorname{ch}_{m}(g)^{(-m,-1)}\right) \wedge \operatorname{ch}_{l}(h)\right) \\
+ & (-1)^{n+m+1} \operatorname{ch}_{n}(f) \Delta\left(\sum_{\substack{1 \leq j \leq m \\
1 \leq k \leq l}} a_{j, k}^{m, l} d \operatorname{ch}_{m}(g)^{(-m+j-1,-j)} \wedge \operatorname{ch}_{l}(h)^{(-l+k-1,-k)}\right)
\end{aligned}
$$

and

$$
\begin{aligned}
& \Phi(h)=(-1)^{n}\left(\operatorname{ch}_{n}(f) \Delta \operatorname{ch}_{m}(g)\right) \wedge\left(\partial \operatorname{ch}_{l}(h)^{(-1,-l)}-\bar{\partial} \operatorname{ch}_{l}(h)^{(-l,-1)}\right) \\
& +(-1)^{n+1} \operatorname{ch}_{n}(f) \Delta\left(\operatorname{ch}_{m}(g) \wedge\left(\partial \operatorname{ch}_{l}(h)^{(-1,-l)}-\bar{\partial} \operatorname{ch}_{l}(h)^{(-l,-1)}\right)\right) \\
& +(-1)^{n} \operatorname{ch}_{n}(f) \Delta\left(\sum_{\substack{1 \leq j \leq m \\
1 \leq k \leq l}} a_{j, k}^{m, l} \operatorname{ch}_{m}(g)^{(-m+j-1,-j)} \wedge d \operatorname{ch}_{l}(h)^{(-l+k-1,-k)}\right) .
\end{aligned}
$$

Let us first calculate $\Phi(f)$. It follows from $d_{\mathcal{D}}\left(\operatorname{ch}_{n}(f)\right)=0$ that $\partial \operatorname{ch}_{n}$ $(f)^{(-n+i-1,-i)}=-\bar{\partial} \operatorname{ch}_{n}(f)^{(-n+i,-i-1)}$ for $1 \leq i \leq n-1$. Then $\Phi(f)$ is expressed as follows:

$$
\begin{aligned}
& \Phi(f)=b_{0, j, k}^{n, m, l} \sum_{\substack{1 \leq j \leq m \\
1 \leq k \leq l}} \bar{\partial} \operatorname{ch}_{n}(f)^{(-n,-1)} \wedge \operatorname{ch}_{m}(g)^{(-m+j-1,-j)} \wedge \operatorname{ch}_{l}(h)^{(-l+k-1,-k)} \\
& +\sum_{\substack{1 \leq i \leq n \\
1 \leq j \leq m \\
1 \leq k \leq l}} b_{i, j, k}^{n, m, l} \partial \operatorname{ch}_{n}(f)^{(-n+i-1,-i)} \wedge \operatorname{ch}_{m}(g)^{(-m+j-1,-j)} \wedge \operatorname{ch}_{l}(h)^{(-l+k-1,-k)}
\end{aligned}
$$

where

$$
\begin{aligned}
b_{0, j, k}^{n, m, l} & =(-1)^{m} a_{j, k}^{m, l}+(-1)^{m+1} a_{j, k}^{n+m, l}+(-1)^{m} a_{j, k}^{n+m, l} \times a_{1, j}^{n, m} \\
& =(-1)^{m} a_{j, k}^{m, l}+2(-1)^{m+1}\left(\begin{array}{c}
n+m \\
n
\end{array}\right)^{-1}\left(\begin{array}{c}
n+m-j \\
n
\end{array}\right) a_{j, k}^{n+m, l}, \\
b_{n, m, k}^{n, m, l} & =(-1)^{m+1} a_{j, k}^{m, l}+(-1)^{m} a_{n+j, k}^{n+m, l}+(-1)^{m} a_{n+j, k}^{n+m, l} \times a_{n, j}^{n, m} \\
& =(-1)^{m+1} a_{j, k}^{m, l}+2(-1)^{m}\left(\begin{array}{c}
n+m \\
n
\end{array}\right)^{-1}\left(\begin{array}{c}
n-j+1 \\
n
\end{array}\right) a_{n+j, k}^{n+m, l},
\end{aligned}
$$

and

$$
\begin{aligned}
b_{i, j, k}^{n, m, l} & =(-1)^{m+1} a_{i+j, k}^{n+m, l} \times a_{i+1, j}^{n, m}+(-1)^{m} a_{i+j, k}^{n+m, l} \times a_{i, j}^{n, m} \\
& =2(-1)^{m}\left(\begin{array}{c}
n+m \\
n
\end{array}\right)^{-1}\left(\begin{array}{c}
n+m-i-j \\
n-i
\end{array}\right)\left(\begin{array}{c}
i+j-1 \\
i
\end{array}\right) a_{i+j, k}^{n+m, l}
\end{aligned}
$$

for $1 \leq i \leq n-1$. 
Lemma 6.20. If $1 \leq j \leq m$ and $1 \leq k \leq l$, then

$$
\left(\begin{array}{c}
n+m \\
n
\end{array}\right)^{-1} \sum_{i=0}^{n}\left(\begin{array}{c}
n+m-i-j \\
n-i
\end{array}\right)\left(\begin{array}{c}
i+j-1 \\
i
\end{array}\right) a_{i+j, k}^{n+m, l}=a_{j, k}^{m, l} .
$$

Proof. By Lemma A.2 and Lemma A.3, we have

$$
\begin{aligned}
& \left(\begin{array}{c}
n+m \\
n
\end{array}\right)^{-1} \sum_{i=0}^{n}\left(\begin{array}{c}
n+m-i-j \\
n-i
\end{array}\right)\left(\begin{array}{c}
i+j-1 \\
i
\end{array}\right) a_{i+j, k}^{n+m, l} \\
& =\left(\begin{array}{c}
n+m \\
n
\end{array}\right)^{-1} \sum_{i=0}^{n}\left(\begin{array}{c}
n+m-i-j \\
n-i
\end{array}\right)\left(\begin{array}{c}
i+j-1 \\
i
\end{array}\right) \\
& -2\left(\begin{array}{c}
n+m \\
n
\end{array}\right)^{-1}\left(\begin{array}{c}
n+m+l \\
n+m
\end{array}\right) \sum_{i=0}^{-1}\left(\begin{array}{c}
n+m-i-j \\
n-i
\end{array}\right)\left(\begin{array}{c}
i+j-1 \\
i
\end{array}\right) \\
& \times \sum_{\alpha=0}^{i+j-1}\left(\begin{array}{c}
n+m+l-i-j-k+1 \\
n+m-\alpha
\end{array}\right)\left(\begin{array}{c}
i+j+k-1 \\
\alpha
\end{array}\right) \\
& =1-2\left(\begin{array}{c}
m+l \\
m
\end{array}\right)^{-1} \sum_{\alpha=0}^{j-1}\left(\begin{array}{c}
m+l-j-k+1 \\
m-\alpha
\end{array}\right)\left(\begin{array}{c}
j+k-1 \\
\alpha
\end{array}\right) \\
& =a_{j, k}^{m, l} .
\end{aligned}
$$

Let

$$
c_{i, j, k}^{n, m}=(-1)^{m} a_{j, k}^{m, l}-2(-1)^{m}\left(\begin{array}{c}
n+m \\
n
\end{array}\right)^{-1} \sum_{\alpha=0}^{i-1}\left(\begin{array}{c}
n+m-\alpha-j \\
n-\alpha
\end{array}\right)\left(\begin{array}{c}
\alpha+j-1 \\
\alpha
\end{array}\right) a_{\alpha+j, k}^{n+m, l} .
$$

Then we have

$$
\begin{aligned}
c_{1, j, k}^{n, m, l} & =b_{0, j, k}^{n, m, l}, \\
c_{i, j, k}^{n, m, l}-c_{i+1, j, k}^{n, m, l} & =b_{i, j, k}^{n, m, l}
\end{aligned}
$$

for $1 \leq i \leq n-1$ and by Lemma 6.20 ,

$$
c_{n, j, k}^{n, m, l}=b_{n, j, k}^{n, m, l} .
$$

Let $\Psi$ be a differential form given by

$$
\Psi=\sum_{\substack{1 \leq i \leq n \\ 1 \leq j \leq m \\ 1 \leq k \leq l}} c_{i, j, k}^{n, m, l} \operatorname{ch}_{n}(f)^{(-n+i-1,-i)} \wedge \operatorname{ch}_{m}(g)^{(-m+j-1,-j)} \wedge \operatorname{ch}_{l}(h)^{(-l+k-1,-k)} .
$$


Lemma 6.21. It follows that $c_{n-i+1, m-j+1, l-k+1}^{n, m, l}=c_{i, j, k}^{n, m, l}$. Hence $\Psi$ is contained in $\mathcal{D}_{n+m+l+2}(X)$.

Proof. We have

$$
\begin{aligned}
c_{n-i+1, m-j+1, l-k+1}^{n, m, l} & \\
= & (-1)^{m} a_{m-j+1, l-k+1}^{m, l} \\
& -2(-1)^{m}\left(\begin{array}{c}
n+m \\
n
\end{array}\right)^{-1} \sum_{\alpha=0}^{n-i}\left(\begin{array}{c}
n-\alpha+j-1 \\
n-\alpha
\end{array}\right)\left(\begin{array}{c}
\alpha+m-j \\
\alpha
\end{array}\right) a_{\alpha+m-j+1, l-k+1}^{n+m, l} \\
= & (-1)^{m+1} a_{j, k}^{m, l}+2(-1)^{m}\left(\begin{array}{c}
n+m \\
n
\end{array}\right)^{-1} \sum_{\alpha=0}^{n-i}\left(\begin{array}{c}
n-\alpha+j-1 \\
n-\alpha
\end{array}\right)\left(\begin{array}{c}
\alpha+m-j \\
\alpha
\end{array}\right) a_{n-\alpha+j, k}^{n+m, l} \\
= & (-1)^{m+1} a_{j, k}^{m, l}+2(-1)^{m}\left(\begin{array}{c}
n+m \\
n
\end{array}\right)^{-1} \sum_{\beta=i}^{n}\left(\begin{array}{c}
\beta+j-1 \\
\beta
\end{array}\right)\left(\begin{array}{c}
n-\beta+m-j \\
n-\beta
\end{array}\right) a_{\beta+j, k}^{n+m, l} .
\end{aligned}
$$

Hence Lemma 6.20 implies that

$$
\begin{aligned}
& c_{n-i+1, m-j+1, l-k+1}^{n, m, l} \\
& =(-1)^{m+1} a_{j, k}^{m, l}+2(-1)^{m} \\
& \times\left(a_{j, k}^{m, l}-\left(\begin{array}{c}
n+m \\
n
\end{array}\right)^{-1} \sum_{\beta=0}^{i-1}\left(\begin{array}{c}
\beta+j-1 \\
\beta
\end{array}\right)\left(\begin{array}{c}
n-\beta+m-j \\
n-\beta
\end{array}\right) a_{\beta+j, k}^{n+m, l}\right) \\
& =(-1)^{m} a_{j, k}^{m, l}-2(-1)^{m}\left(\begin{array}{c}
n+m \\
n
\end{array}\right)^{-1} \sum_{\beta=0}^{i-1}\left(\begin{array}{c}
n+m-\beta-j \\
n-\beta
\end{array}\right)\left(\begin{array}{c}
\beta+j-1 \\
\beta
\end{array}\right) a_{\beta+j, k}^{n+m, l} \\
& =c_{i, j, k}^{n, m, l} \text {. }
\end{aligned}
$$

Let us denote the parts of $d \Psi$ including the derivatives of $\operatorname{ch}_{n}(f), \operatorname{ch}_{m}(g)$ and $\operatorname{ch}_{l}(h)$ by $\Psi(f), \Psi(g)$ and $\Psi(h)$ respectively. Then $d \Psi=\Psi(f)+\Psi(g)+\Psi(h)$ and

$$
\begin{aligned}
\Psi(f)= & \sum_{\substack{1 \leq i \leq n \\
1 \leq j \leq m \\
1 \leq k \leq l}} c_{i, j, k}^{n, m, l} d \operatorname{ch}_{n}(f)^{(-n+i-1,-i)} \\
& \wedge \operatorname{ch}_{m}(g)^{(-m+j-1,-j)} \wedge \operatorname{ch}_{l}(h)^{(-l+k-1,-k)}
\end{aligned}
$$




$$
\begin{aligned}
& =\sum_{\substack{1 \leq j \leq m \\
1 \leq k \leq l}} c_{1, j, k}^{n, m, l} \bar{\partial} \operatorname{ch}_{n}(f)^{(-n,-1)} \wedge \operatorname{ch}_{m}(g)^{(-m+j-1,-j)} \wedge \operatorname{ch}_{l}(h)^{(-l+k-1,-k)} \\
& +\sum_{\substack{1 \leq i \leq n-1 \\
1 \leq j \leq m \\
1 \leq k \leq l}}\left(c_{i, j, k}^{n, m, l}-c_{i+1, j, k}^{n, m, l}\right) \partial \operatorname{ch}_{n}(f)^{(-n+i-1,-i)} \\
& +\sum_{\substack{1 \leq j \leq m \\
1 \leq k \leq l}} c_{n, j, k}^{n, m, l} \partial \operatorname{ch}_{n}(f)^{(-1,-n)} \wedge \operatorname{ch}_{m}(g)^{(-m+j-1,-j)} \wedge \operatorname{ch}_{l}(h)^{(-l+k-1,-k)} \\
& =\Phi(f) .
\end{aligned}
$$

Let us express $\Phi(h)-\Psi(h)$ as follows:

$$
\begin{aligned}
& \Phi(h)-\Psi(h) \\
& =d_{i, j, 0}^{n, m, l} \sum_{\substack{1 \leq i \leq n \\
1 \leq j \leq m}} \operatorname{ch}_{n}(f)^{(-n+i-1,-i)} \wedge \operatorname{ch}_{m}(g)^{(-m+j-1,-j)} \wedge \bar{\partial} \operatorname{ch}_{l}(h)^{(-l,-1)} \\
& \quad+\sum_{\substack{1 \leq i \leq n \\
1 \leq j \leq m \\
1 \leq k \leq l}} d_{i, j, k}^{n, m, l} \operatorname{ch}_{n}(f)^{(-n+i-1,-i)} \wedge \operatorname{ch}_{m}(g)^{(-m+j-1,-j)} \wedge \partial \operatorname{ch}_{l}(h)^{(-l+k-1,-k)} .
\end{aligned}
$$

Lemma 6.22. It follows that $d_{i, j, k}^{n, m, l}=0$, therefore $\Phi(h)-\Psi(h)=0$.

Proof. When $1 \leq k \leq l-1$,

$$
\begin{aligned}
d_{i, j, k}^{n, m, l}= & (-1)^{n} a_{i, j+k}^{n, m+l} \times\left(a_{j, k}^{m, l}-a_{j, k+1}^{m, l}\right)-(-1)^{n+m}\left(c_{i, j, k}^{n, m, l}-c_{i, j, k+1}^{n, m, l}\right) \\
= & (-1)^{n+1}\left(1-a_{i, j+k}^{n, m+l}\right)\left(a_{j, k}^{m, l}-a_{j, k+1}^{m, l}\right) \\
& +2(-1)^{n}\left(\begin{array}{c}
n+m \\
n
\end{array}\right)-1 \sum_{\alpha=0}^{i-1}\left(\begin{array}{c}
n+m-\alpha-j \\
n-\alpha
\end{array}\right)\left(\begin{array}{c}
\alpha+j-1 \\
\alpha
\end{array}\right)\left(a_{\alpha+j, k}^{n+m, l}-a_{\alpha+j, k+1}^{n+m, l}\right) \\
= & 4(-1)^{n}\left(\begin{array}{c}
n+m+l \\
n
\end{array}\right)-1\left(\begin{array}{c}
m+l \\
l
\end{array}\right)-1\left(\begin{array}{c}
m+l-j-k \\
m-j
\end{array}\right)\left(\begin{array}{c}
j+k-1 \\
j-1
\end{array}\right) \\
& \times \sum_{\alpha=0}^{i-1}\left(\begin{array}{c}
n+m+l-i-j-k+1 \\
n-\alpha
\end{array}\right)\left(\begin{array}{c}
i+j+k-1 \\
\alpha
\end{array}\right) \\
& \left.-4(-1)^{n}\left(\begin{array}{c}
n+m+l \\
l
\end{array}\right) \begin{array}{c}
-1 \\
n+m \\
n+1
\end{array}\right) \\
& \times \sum_{\alpha=0}^{i-1}\left(\begin{array}{c}
n+m+l-\alpha-j-k \\
n+m-\alpha-j
\end{array}\right)\left(\begin{array}{c}
\alpha+j+k-1 \\
\alpha+j-1
\end{array}\right)\left(\begin{array}{c}
n+m-\alpha-j \\
n-\alpha
\end{array}\right)\left(\begin{array}{c}
\alpha+j-1 \\
\alpha
\end{array}\right) .
\end{aligned}
$$


Since

$$
\begin{aligned}
\left(\begin{array}{c}
n+m+l \\
l
\end{array}\right)\left(\begin{array}{c}
n+m \\
n
\end{array}\right) & =\left(\begin{array}{c}
n+m+l \\
n
\end{array}\right)\left(\begin{array}{c}
m+l \\
l
\end{array}\right), \\
\left(\begin{array}{c}
n+m+l-\alpha-j-k \\
n+m-\alpha-j
\end{array}\right)\left(\begin{array}{c}
n+m-\alpha-j \\
n-\alpha
\end{array}\right) & =\left(\begin{array}{c}
n+m+l-\alpha-j-k \\
m+l-j-k
\end{array}\right)\left(\begin{array}{c}
m+l-j-k \\
m-j
\end{array}\right), \\
\left(\begin{array}{c}
\alpha+j+k-1 \\
\alpha+j-1
\end{array}\right)\left(\begin{array}{c}
\alpha+j-1 \\
\alpha
\end{array}\right) & =\left(\begin{array}{c}
\alpha+j+k-1 \\
\alpha
\end{array}\right)\left(\begin{array}{c}
j+k-1 \\
j-1
\end{array}\right),
\end{aligned}
$$

we have

$$
\begin{aligned}
d_{i, j, k}^{n, m, l}= & 4(-1)^{n}\left(\begin{array}{c}
n+m+l \\
n
\end{array}\right)^{-1}\left(\begin{array}{c}
m+l \\
l
\end{array}\right)^{-1}\left(\begin{array}{c}
m+l-j-k \\
m-j
\end{array}\right)\left(\begin{array}{c}
j+k-1 \\
j-1
\end{array}\right) \\
& \times\left(\sum_{\alpha=0}^{i-1}\left(\begin{array}{c}
n+m+l-i-j-k+1 \\
n-\alpha
\end{array}\right)\left(\begin{array}{c}
i+j+k-1 \\
\alpha
\end{array}\right)-\sum_{\alpha=0}^{i-1}\left(\begin{array}{c}
n+m+l-\alpha-j-k \\
n-\alpha
\end{array}\right)\left(\begin{array}{c}
\alpha+j+k-1 \\
\alpha
\end{array}\right)\right) \\
= & 0
\end{aligned}
$$

by Lemma A.3. When $k=0$,

$$
\begin{aligned}
d_{i, j, 0}^{n, m, l}= & (-1)^{n+1} a_{i, j}^{n, m}+(-1)^{n} a_{i, j}^{n, m+l} \times\left(1+a_{j, 1}^{m, l}\right)-(-1)^{n+m} c_{i, j, 1}^{n, m, l} \\
= & (-1)^{n+1}\left(\left(1-2\left(\begin{array}{c}
n+m \\
n
\end{array}\right)^{-1} \sum_{\alpha=0}^{i-1}\left(\begin{array}{c}
n+m-i-j+1 \\
n-\alpha
\end{array}\right)\left(\begin{array}{c}
i+j-1 \\
\alpha
\end{array}\right)\right)\right. \\
& +2(-1)^{n}\left(\begin{array}{c}
m+l \\
m
\end{array}\right)-1\left(\begin{array}{c}
m+l-j \\
l
\end{array}\right) \\
& \times\left(1-2\left(\begin{array}{c}
n+m+l \\
n
\end{array}\right)^{-1} \sum_{\alpha=0}^{i-1}\left(\begin{array}{c}
n+m+l-i-j+1 \\
n-\alpha
\end{array}\right)\left(\begin{array}{c}
i+j-1 \\
\alpha
\end{array}\right)\right) \\
& -(-1)^{n}\left(\begin{array}{c}
-1+2\left(\begin{array}{c}
m+l \\
l
\end{array}\right) \\
-1
\end{array}\left(\begin{array}{c}
m+l-j \\
l
\end{array}\right)\right) \\
+ & 2(-1)^{n}\left(\begin{array}{c}
n+m \\
n
\end{array}\right)-1 \\
& \times\left(-1+2\left(\begin{array}{c}
n+m+l \\
l
\end{array} \sum_{\alpha=0}^{-1}\left(\begin{array}{c}
n+m-\alpha-j \\
n-\alpha
\end{array}\right)\left(\begin{array}{c}
\alpha+j-1 \\
\alpha+l-\alpha-j
\end{array}\right)\right) .\right.
\end{aligned}
$$

Since

$$
\left(\begin{array}{c}
n+m+l-\alpha-j \\
l
\end{array}\right)\left(\begin{array}{c}
n+m-\alpha-j \\
n-\alpha
\end{array}\right)=\left(\begin{array}{c}
n+m+l-\alpha-j \\
n-\alpha
\end{array}\right)\left(\begin{array}{c}
m+l-j \\
l
\end{array}\right),
$$

we have

$$
\begin{aligned}
d_{i, j, 0}^{n, m, l}=2(-1)^{n} & \left(\begin{array}{c}
n+m \\
n
\end{array}\right)^{-1} \\
& \times\left(\sum_{\alpha=0}^{i-1}\left(\begin{array}{c}
n+m-i-j+1 \\
n-\alpha
\end{array}\right)\left(\begin{array}{c}
i+j-1 \\
\alpha
\end{array}\right)-\sum_{\alpha=0}^{i-1}\left(\begin{array}{c}
n+m-\alpha-j \\
n-\alpha
\end{array}\right)\left(\begin{array}{c}
\alpha+j-1 \\
\alpha
\end{array}\right)\right) \\
+ & 4(-1)^{n+1}\left(\begin{array}{c}
n+m+l \\
n
\end{array}\right)-1\left(\begin{array}{c}
m+l \\
l
\end{array}\right)-1\left(\begin{array}{c}
m+l-j \\
l
\end{array}\right) \\
& \times\left(\sum_{\alpha=0}^{i-1}\left(\begin{array}{c}
n+m+l-i-j+1 \\
n-\alpha
\end{array}\right)\left(\begin{array}{c}
i+j-1 \\
\alpha
\end{array}\right)-\sum_{\alpha=0}^{i-1}\left(\begin{array}{c}
n+m+l-\alpha-j \\
n-\alpha
\end{array}\right)\left(\begin{array}{c}
\alpha+j-1 \\
\alpha
\end{array}\right)\right)
\end{aligned}
$$




$$
=0
$$

by Lemma A.3. We can prove that $d_{i, j, l}^{n, m}=0$ in the same way.

We finally calculate $\Phi(g)-\Psi(g)$. Let us express it as follows:

$$
\begin{gathered}
\Phi(g)-\Psi(g) \\
=e_{i, 0, k}^{n, m, l} \sum_{\substack{1 \leq i \leq n \\
1 \leq k \leq l}} \operatorname{ch}_{n}(f)^{(-n+i-1,-i)} \wedge \bar{\partial} \operatorname{ch}_{m}(g)^{(-m,-1)} \wedge \operatorname{ch}_{l}(h)^{(-l+k-1,-k)} \\
+\sum_{\substack{1 \leq i \leq n \\
1 \leq j \leq m \\
1 \leq k \leq l}} e_{i, j, k}^{n, m, l} \operatorname{ch}_{n}(f)^{(-n+i-1,-i)} \wedge \partial \operatorname{ch}_{m}(g)^{(-m+j-1,-j)} \\
\quad \wedge \operatorname{ch}_{l}(h)^{(-l+k-1,-k)} .
\end{gathered}
$$

Lemma 6.23. When $1 \leq j \leq m-1, e_{i, j, k}^{n, m, l}=0$ and $e_{i, 0, k}^{n, m, l}=e_{i, m, k}^{n, m, l}=$ $(-1)^{n+m+1}$.

Proof. When $1 \leq j \leq m-1$,

$$
\begin{aligned}
e_{i, j, k}^{n, m, l}= & (-1)^{n+m+1} a_{i+j, k}^{n+m, l} \times\left(a_{i, j}^{n, m}-a_{i, j+1}^{n, m}\right) \\
& +(-1)^{n+m+1} a_{i, j+k}^{n, m+l} \times\left(a_{j, k}^{m, l}-a_{j+1, k}^{m, l}\right) \\
& +(-1)^{n}\left(c_{i, j, k}^{n, m, l}-c_{i, j, m+1, k}^{n, m}\right) \\
= & (-1)^{n+m+1} a_{i+j, k}^{n+m, l} \times\left(a_{i, j}^{n, m}-a_{i, j+1}^{n, m}\right) \\
& +(-1)^{n+m+1}\left(a_{i, j+k}^{n, m+l}-1\right) \times\left(a_{j, k}^{m, l}-a_{j+1, k}^{m, l}\right) \\
& +2(-1)^{n+m+1}\left(\begin{array}{c}
n+m \\
n
\end{array}\right)^{-1} \sum_{\alpha=0}^{i-1}\left(\begin{array}{c}
n+m-\alpha-j \\
n-\alpha
\end{array}\right)\left(\begin{array}{c}
\alpha+j-1 \\
\alpha
\end{array}\right) a_{\alpha+j, k}^{n+m, l} \\
& -2(-1)^{n+m+1}\left(\begin{array}{c}
n+m \\
n
\end{array}\right)^{-1} \sum_{\alpha=0}^{i-1}\left(\begin{array}{c}
n+m-\alpha-j-1 \\
n-\alpha
\end{array}\right)\left(\begin{array}{c}
\alpha+j \\
\alpha
\end{array}\right) a_{\alpha+j+1, k}^{n+m, l} .
\end{aligned}
$$

Hence we have

$$
\begin{aligned}
& e_{i+1, j, k}^{n, m, l}-e_{i, j, k}^{n, m, l} \\
&=(-1)^{n+m+1} a_{i+j+1, k}^{n+m, l} \times\left(a_{i+1, j}^{n, m}-a_{i+1, j+1}^{n, m}\right) \\
&-(-1)^{n+m+1} a_{i+j, k}^{n+m, l} \times\left(a_{i, j}^{n, m}-a_{i, j+1}^{n, m}\right) \\
&+(-1)^{n+m+1}\left(a_{i+1, j+k}^{n, m+l}-a_{i, j+k}^{n, m+l}\right)\left(a_{j, k}^{m, l}-a_{j+1, k}^{m, l}\right) \\
&+2(-1)^{n+m+1}\left(\begin{array}{c}
n+m \\
n
\end{array}\right)^{-1}\left(\left(\begin{array}{c}
n+m-i-j \\
n-i
\end{array}\right)\left(\begin{array}{c}
i+j-1 \\
i
\end{array}\right) a_{i+j, k}^{n+m, l}\right.
\end{aligned}
$$




$$
\begin{aligned}
& \left.-\left(\begin{array}{c}
n+m-i-j-1 \\
n-i
\end{array}\right)\left(\begin{array}{c}
i+j \\
i
\end{array}\right) a_{i+j+1, k}^{n+m, l}\right) \\
& =2(-1)^{n+m}\left(\begin{array}{c}
n+m \\
n
\end{array}\right)^{-1}\left(\begin{array}{c}
n+m-i-j-1 \\
n-i-1
\end{array}\right)\left(\begin{array}{c}
i+j \\
i
\end{array}\right) a_{i+j+1, k}^{n+m, l} \\
& -2(-1)^{n+m}\left(\begin{array}{c}
n+m \\
n
\end{array}\right)^{-1}\left(\begin{array}{c}
n+m-i-j \\
n-i
\end{array}\right)\left(\begin{array}{c}
i+j-1 \\
i-1
\end{array}\right) a_{i+j, k}^{n+m, l} \\
& +(-1)^{n+m+1}\left(a_{i+1, j+k}^{n, m+l}-a_{i, j+k}^{n, m+l}\right)\left(a_{j, k}^{m, l}-a_{j+1, k}^{m, l}\right) \\
& +2(-1)^{n+m+1}\left(\begin{array}{c}
n+m \\
n
\end{array}\right)^{-1}\left(\left(\begin{array}{c}
n+m-i-j \\
n-i
\end{array}\right)\left(\begin{array}{c}
i+j-1 \\
i
\end{array}\right) a_{i+j, k}^{n+m, l}\right. \\
& \left.-\left(\begin{array}{c}
n+m-i-j-1 \\
n-i
\end{array}\right)\left(\begin{array}{c}
i+j \\
i
\end{array}\right) a_{i+j+1, k}^{n+m, l}\right) \\
& =2(-1)^{n+m}\left(\begin{array}{c}
n+m \\
n
\end{array}\right)^{-1}\left(\begin{array}{c}
n+m-i-j \\
n-i
\end{array}\right)\left(\begin{array}{c}
i+j \\
i
\end{array}\right)\left(a_{i+j+1, k}^{n+m, l}-a_{i+j, k}^{n+m, l}\right) \\
& +(-1)^{n+m+1}\left(a_{i+1, j+k}^{n, m+l}-a_{i, j+k}^{n, m+l}\right)\left(a_{j, k}^{m, l}-a_{j+1, k}^{m, l}\right) \\
& =-4(-1)^{n+m}\left(\begin{array}{c}
n+m \\
n
\end{array}\right)^{-1}\left(\begin{array}{c}
n+m-i-j \\
n-i
\end{array}\right)\left(\begin{array}{c}
i+j \\
i
\end{array}\right)\left(\begin{array}{c}
n+m+l \\
n+m
\end{array}\right)-1\left(\begin{array}{c}
n+m+l-i-j-k \\
n+m-i-j
\end{array}\right)\left(\begin{array}{c}
i+j+k-1 \\
i+j
\end{array}\right) \\
& +4(-1)^{n+m}\left(\begin{array}{c}
n+m+l \\
n
\end{array}\right)^{-1}\left(\begin{array}{c}
n+m+l-i-j-k \\
n-i
\end{array}\right)\left(\begin{array}{c}
i+j+k-1 \\
i
\end{array}\right)\left(\begin{array}{c}
m+l \\
m
\end{array}\right)-1\left(\begin{array}{c}
m+l-j-k \\
m-j
\end{array}\right)\left(\begin{array}{c}
j+k-1 \\
j
\end{array}\right) \\
& =0
\end{aligned}
$$

and

$$
\begin{aligned}
e_{1, j, k}^{n, m, l}= & (-1)^{n+m+1} a_{j+1, k}^{n+m, l} \times\left(a_{1, j}^{n, m}-a_{1, j+1}^{n, m}\right) \\
& +(-1)^{n+m+1}\left(a_{1, j+k}^{n, m+l}-1\right)\left(a_{j, k}^{m, l}-a_{j+1, k}^{m, l}\right) \\
& +2(-1)^{n+m+1}\left(\begin{array}{c}
n+m \\
n
\end{array}\right)-1\left(\left(\begin{array}{c}
n+m-j \\
n
\end{array}\right) a_{j, k}^{n+m, l}-\left(\begin{array}{c}
n+m-j-1 \\
n
\end{array}\right) a_{j+1, k}^{n+m, l}\right) \\
= & (-1)^{n+m+1}\left(\begin{array}{c}
\left.a_{1, j+k}^{n, m+l}-1\right)\left(a_{j, k}^{m, l}-a_{j+1, k}^{m, l}\right.
\end{array}\right) \\
& -2(-1)^{n+m}\left(\begin{array}{c}
n+m \\
n
\end{array}\right)^{-1}\left(\begin{array}{c}
n+m-j \\
n
\end{array}\right)\left(a_{j, k}^{n+m, l}-a_{j+1, k}^{n+m, l}\right) \\
= & \left.4(-1)^{n+m}\left(\begin{array}{c}
n+m+l \\
n
\end{array}\right)^{-1}\left(\begin{array}{c}
n+m+l-j-k \\
n
\end{array}\right)\left(\begin{array}{c}
m+l \\
m
\end{array}\right) \begin{array}{c}
m+l-j-k \\
m-j
\end{array}\right)\left(\begin{array}{c}
j+k-1 \\
j
\end{array}\right) \\
& -4(-1)^{n+m}\left(\begin{array}{c}
n+m \\
n
\end{array}\right)^{-1}\left(\begin{array}{c}
n+m-j \\
n
\end{array}\right)\left(\begin{array}{c}
n+m+l \\
n+m
\end{array}\right) \\
= & 0 .
\end{aligned}
$$

Hence $e_{i, j, k}^{n, m, l}=0$ if $1 \leq j \leq m-1$. On the other hand, when $j=0$,

$$
\begin{aligned}
e_{i, 0, k}^{n, m, l}= & (-1)^{n+m+1} a_{i, k}^{n+m, l} \times\left(1+a_{i, 1}^{n, m}\right)+(-1)^{n+m} a_{i, k}^{n, m+l} \\
& \times\left(1-a_{1, k}^{m, l}\right)-(-1)^{n+1} c_{i, 1, k}^{n, m, l} \\
= & 2(-1)^{n+m+1}\left(\begin{array}{c}
n+m \\
n
\end{array}\right)^{-1}\left(\begin{array}{c}
n+m-i \\
m
\end{array}\right) a_{i, k}^{n+m, l} \\
& +2(-1)^{n+m}\left(\begin{array}{c}
m+l \\
m
\end{array}\right)^{-1}\left(\begin{array}{c}
m+l-k \\
m
\end{array}\right) a_{i, k}^{n, m+l} \\
& +(-1)^{n+m} a_{1, k}^{m, l}-2(-1)^{n+m}\left(\begin{array}{c}
n+m \\
n
\end{array}\right)^{-1} \sum_{\alpha=0}^{i-1}\left(\begin{array}{c}
n+m-\alpha-1 \\
n-\alpha
\end{array}\right) a_{\alpha+1, k}^{n+m, l} .
\end{aligned}
$$


Hence we have

$$
\begin{aligned}
e_{i+1,0, k}^{n, m, l} & -e_{i, 0, k}^{n, m, l} \\
= & -2(-1)^{n+m}\left(\begin{array}{c}
n+m \\
n
\end{array}\right)^{-1}\left(\begin{array}{c}
n+m-i-1 \\
m
\end{array}\right) a_{i+1, k}^{n+m, l} \\
& +2(-1)^{n+m}\left(\begin{array}{c}
n+m \\
n
\end{array}\right)^{-1}\left(\begin{array}{c}
n+m-i \\
m
\end{array}\right) a_{i, k}^{n+m, l} \\
& +2(-1)^{n+m}\left(\begin{array}{c}
m+l \\
m
\end{array}\right)-1\left(\begin{array}{c}
m+l-k \\
m
\end{array}\right)\left(a_{i+1, k}^{n, m+l}-a_{i, k}^{n, m+l}\right) \\
& -2(-1)^{n+m}\left(\begin{array}{c}
n+m \\
n
\end{array}\right)^{-1}\left(\begin{array}{c}
n+m-i-1 \\
n-i
\end{array}\right) a_{i+1, k}^{n+m, l} \\
= & 2(-1)^{n+m+1}\left(\begin{array}{c}
n+m \\
n
\end{array}\right)-\left(\begin{array}{c}
n+m-i \\
m
\end{array}\right)\left(a_{i+1, k}^{n+m, l}-a_{i, k}^{n+m, l}\right) \\
& +2(-1)^{n+m}\left(\begin{array}{c}
m+l \\
m
\end{array}\right){ }^{-1}\left(\begin{array}{c}
m+l-k \\
m
\end{array}\right)\left(a_{i+1, k}^{n, m+l}-a_{i, k}^{n, m+l}\right) \\
= & 4(-1)^{n+m}\left(\begin{array}{c}
n+m \\
n
\end{array}\right)^{-1}\left(\begin{array}{c}
n+m-i \\
m
\end{array}\right)\left(\begin{array}{c}
n+m+l \\
n+m
\end{array}\right)-1 \\
& -4(-1)^{n+m}\left(\begin{array}{c}
m+m+l-i-k \\
n+m-i
\end{array}\right)\left(\begin{array}{c}
i+k-1 \\
m
\end{array}\right) \\
= & 0
\end{aligned}
$$

and

$$
\begin{aligned}
& e_{1,0, k}^{n, m}=2(-1)^{n+m+1}\left(\begin{array}{c}
n+m \\
n
\end{array}\right)^{-1}\left(\begin{array}{c}
n+m-1 \\
m
\end{array}\right) a_{1, k}^{n+m, l} \\
& +2(-1)^{n+m}\left(\begin{array}{c}
m+l \\
m
\end{array}\right)^{-1}\left(\begin{array}{c}
m+l-k \\
m
\end{array}\right) a_{1, k}^{n, m+l} \\
& +(-1)^{n+m} a_{1, k}^{m, l}-2(-1)^{n+m}\left(\begin{array}{c}
n+m \\
n
\end{array}\right)^{-1}\left(\begin{array}{c}
n+m-1 \\
n
\end{array}\right) a_{1, k}^{n+m, l} \\
& =2(-1)^{n+m+1}\left(1-2\left(\begin{array}{c}
n+m+l \\
n+m
\end{array}\right)^{-1}\left(\begin{array}{c}
n+m+l-k \\
n+m
\end{array}\right)\right) \\
& +(-1)^{n+m}\left(1-2\left(_{m}^{m+l}\right)^{-1}\left(\begin{array}{c}
m+l-k \\
m
\end{array}\right)\right) \\
& +2(-1)^{n+m}\left(\begin{array}{c}
m+l \\
m
\end{array}\right)^{-1}\left(\begin{array}{c}
m+l-k \\
m
\end{array}\right)\left(1-2\left(\begin{array}{c}
n+m+l \\
n
\end{array}\right)^{-1}\left(\begin{array}{c}
n+m+l-k \\
n
\end{array}\right)\right) \\
& =(-1)^{n+m+1}+4(-1)^{n+m}\left(\begin{array}{c}
n+m+l \\
n+m
\end{array}\right)^{-1}\left(\begin{array}{c}
n+m+l-k \\
n+m
\end{array}\right) \\
& -4(-1)^{n+m}\left(\begin{array}{c}
m+l \\
m
\end{array}\right)-1\left(\begin{array}{c}
m+l-k \\
m
\end{array}\right)\left(\begin{array}{c}
n+m+l \\
n
\end{array}\right)^{-1}\left(\begin{array}{c}
n+m+l-k \\
n
\end{array}\right) \\
& =(-1)^{n+m+1} \text {. }
\end{aligned}
$$

Hence $e_{i, 0, k}^{n, m, l}=(-1)^{n+m+1}$. We can prove that $e_{i, m, k}^{n, m, l}=(-1)^{n+m+1}$ in the same way.

Let us return to the proof of Proposition 6.17. By the above calculations, 
we have

$$
\begin{aligned}
\Phi & -d \Psi \\
= & (-1)^{n+m+1} \sum_{\substack{1 \leq i \leq n \\
1 \leq k \leq l}} \operatorname{ch}_{n}(f)^{(-n+i-1,-i)} \\
& \wedge\left(\bar{\partial} \operatorname{ch}_{m}(g)^{(-m,-1)}+\partial \operatorname{ch}_{m}(g)^{(-1,-m)}\right) \wedge \operatorname{ch}_{l}(h)^{(-l+k-1,-k)} \\
= & (-1)^{n+m+1} \operatorname{ch}_{n}(f) \wedge d \operatorname{ch}_{m}(g) \wedge \operatorname{ch}_{l}(h) .
\end{aligned}
$$

Since $\Psi \in \mathcal{D}_{n+m+l+2}(X)$ and $d_{\mathcal{D}} \Psi=-d \Psi$, we have completed the proof.

\section{$\S 7 . \quad$ Direct Images}

\section{§7.1. Higher analytic torsion forms}

We start this section by recalling the higher analytic torsion forms defined by Bismut and Köhler [2]. We fix some notations.

Let $\varphi: M \rightarrow N$ be a smooth projective morphism of compact complex algebraic manifolds. Let $T \varphi$ be the relative tangent bundle of $\varphi$ and we fix a smooth hermitian metric $h_{\varphi}$ on $T \varphi$ that induces a Kähler metric on each fiber $\varphi^{-1}(y)$ for $y \in N$. The pair $\left(\varphi, h_{\varphi}\right)$ is called a Kähler fibration. A real closed $(1,1)$-form $\Omega$ on $M$ is called a Kähler form with respect to $h_{\varphi}$ if the restriction of $\Omega$ to $\varphi^{-1}(y)$ is an associated Kähler form. Let us write $\overline{T \varphi}=\left(T \varphi, h_{\varphi}\right)$ and denote by $\operatorname{Td}(\overline{T \varphi})$ the Todd polynomial for $\overline{T \varphi}$.

Let $\bar{E}$ be a $\varphi$-acyclic hermitian vector bundle on $M$, that is, $\bar{E}$ is a hermitian vector bundle on $M$ such that the higher direct image $R^{i} \varphi_{*} \bar{E}$ is trivial for $i>0$. Then the direct image $\varphi_{*} \bar{E}$ becomes a vector bundle and is equipped with the $L_{2}$-hermitian metric. The Grothendieck-Riemann-Roch theorem says that the two closed forms

$$
\frac{1}{(2 \pi \sqrt{-1})^{\operatorname{dim}(M / N)}} \int_{M / N} \operatorname{Td}(\overline{T \varphi}) \operatorname{ch}_{0}(\bar{E}) \text { and } \operatorname{ch}_{0}\left(\varphi_{*} \bar{E}\right)
$$

give the same cohomology class. The higher analytic torsion form $T(\bar{E}, \varphi, \Omega) \in$ $\mathcal{D}_{1}(N)$ is a homotopy between these forms, namely,

$$
d_{\mathcal{D}} T(\bar{E}, \varphi, \Omega)=\operatorname{ch}_{0}\left(\varphi_{*} \bar{E}\right)-\frac{1}{(2 \pi \sqrt{-1})^{\operatorname{dim}(M / N)}} \int_{M / N} \operatorname{Td}(\overline{T \varphi}) \operatorname{ch}_{0}(\bar{E}) .
$$

Dependence of $T(\bar{E}, \varphi, \Omega)$ on a Kähler form has been discussed in [2]. Following their argument, for two Kähler forms $\Omega$ and $\Omega^{\prime}$ giving the same 
hermitian metric on $T \varphi$, we can obtain $\mu\left(\bar{E}, \Omega, \Omega^{\prime}\right) \in \mathcal{D}_{2}(N)$ such that

$$
d_{\mathcal{D}} \mu\left(\bar{E}, \Omega, \Omega^{\prime}\right)=T(\bar{E}, \varphi, \Omega)-T\left(\bar{E}, \varphi, \Omega^{\prime}\right) .
$$

Finally let us discuss the compatibility of $T(\bar{E}, \varphi, \Omega)$ and $\mu\left(\bar{E}, \Omega, \Omega^{\prime}\right)$ with the pull back for a closed immersion. Consider the following cartesian square:

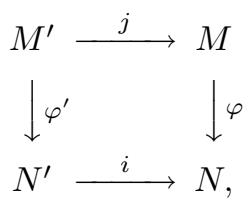

where $i$ and $j$ are closed immersions and $\varphi$ is a Kähler fibration with respect to a smooth hermitian metric $h_{\varphi}$ on $T \varphi$. Then it follows that $T \varphi^{\prime} \simeq j^{*} T \varphi$, therefore a hermitian metric $h_{\varphi^{\prime}}$ on $T \varphi^{\prime}$ with which $\varphi^{\prime}$ becomes a Kähler fibration is induced from $h_{\varphi}$. If $\Omega$ is a Kähler form with respect to $h_{\varphi}$, then $j^{*} \Omega$ is a Kähler form with respect to $h_{\varphi^{\prime}}$.

Take a $\varphi$-acyclic hermitian vector bundle $\bar{E}$ on $M$. Then it is obvious that the ingredients of the definitions of $T(\bar{E}, \varphi, \Omega)$ and $\mu\left(\bar{E}, \Omega, \Omega^{\prime}\right)$ such as the Bismut superconnection and the number operator are compatible with the pull back for the immersions $i$ and $j$. Hence we have

$$
\begin{aligned}
i^{*} T(\bar{E}, \varphi, \Omega) & =T\left(j^{*} \bar{E}, \varphi^{\prime}, j^{*} \Omega\right), \\
i^{*} \mu\left(\bar{E}, \Omega, \Omega^{\prime}\right) & =\mu\left(j^{*} \bar{E}, j^{*} \Omega, j^{*} \Omega^{\prime}\right) .
\end{aligned}
$$

\section{$\S 7.2$. Higher analytic torsion forms for cubes}

In this subsection we introduce the higher analytic torsion form of an exact hermitian $n$-cube defined by Roessler [13].

Let $\varphi: M \rightarrow N, T \varphi$ and $h_{\varphi}$ be as in the previous subsection. Let $\mathcal{F}$ be an exact hermitian $n$-cube made of $\varphi$-acyclic vector bundles on $M$. Then $\lambda \mathcal{F}$ is also made of $\varphi$-acyclic vector bundles and there is a canonical isomorphism $\varphi_{*}\left(\operatorname{tr}_{n} \lambda \mathcal{F}\right) \simeq \lambda \operatorname{tr}_{n} \varphi_{*} \mathcal{F}$. When we put the $L_{2}$-metrics on the both sides, however, this isomorphism does not preserve the metrics. In [13, §3.1], Roessler has constructed a hermitian vector bundle connecting these metrics. Namely, he has defined a hermitian vector bundle $\overline{h(\mathcal{F})}$ on $N \times\left(\mathbb{P}^{1}\right)^{n+1}$ satisfying the following conditions:

$$
\begin{aligned}
&\left.\overline{h(\mathcal{F})}\right|_{X \times\{0\} \times\left(\mathbb{P}^{1}\right)^{n}}=\varphi_{*}\left(\operatorname{tr}_{n} \lambda \mathcal{F}\right), \\
&\left.\overline{h(\mathcal{F})}\right|_{X \times\{\infty\} \times\left(\mathbb{P}^{1}\right)^{n}}=\lambda \operatorname{tr}_{n} \varphi_{*} \mathcal{F}
\end{aligned}
$$


and

$$
\begin{aligned}
&\left.\overline{h(\mathcal{F})}\right|_{X \times\left(\mathbb{P}^{1}\right)^{i} \times\{0\} \times\left(\mathbb{P}^{1}\right)^{n-i}}=\overline{h\left(\partial_{i}^{0} \mathcal{F}\right)}, \\
&\left.\overline{h(\mathcal{F})}\right|_{X \times\left(\mathbb{P}^{1}\right)^{i} \times\{\infty\} \times\left(\mathbb{P}^{1}\right)^{n-i}}=\overline{h\left(\partial_{i}^{-1} \mathcal{F}\right)} \oplus \overline{h\left(\partial_{i}^{1} \mathcal{F}\right)}
\end{aligned}
$$

for $1 \leq i \leq n$. Let us write

$$
\begin{aligned}
& T_{1}(\mathcal{F}, \varphi) \\
& \quad=\frac{(-1)^{n}}{2(2 \pi \sqrt{-1})^{n+1}(n+1) !} \int_{\left(\mathbb{P}^{1}\right)^{n+1}} \operatorname{ch}_{0}(\overline{h(\mathcal{F})}) \sum_{i=1}^{n+1}(-1)^{i} S_{n+1}^{i} \in \mathcal{D}_{n+1}(N) .
\end{aligned}
$$

Take a Kähler form $\Omega$ with respect to $h_{\varphi}$ and

$$
T_{2}(\mathcal{F}, \varphi, \Omega)=\frac{(-1)^{n+1}}{(2 \pi \sqrt{-1})^{n}(n+1) !} \int_{\left(\mathbb{P}^{1}\right)^{n}} \sum_{i=1}^{n+1}(-1)^{i} S_{n+1}^{i}(\mathcal{F}) \in \mathcal{D}_{n+1}(N)
$$

where

$$
S_{n+1}^{i}(\mathcal{F})=S_{n+1}^{i}\left(T\left(\operatorname{tr}_{n} \lambda \mathcal{F}, \varphi, \Omega\right), \log \left|z_{1}\right|^{2}, \ldots, \log \left|z_{n}\right|^{2}\right) .
$$

Theorem 7.1 ([13, Thm. 3.6]). We have

$$
\begin{aligned}
& d_{\mathcal{D}} T_{1}(\mathcal{F}, \varphi)+T_{1}(\partial \mathcal{F}, \varphi) \\
& \quad=\operatorname{ch}_{n}\left(\varphi_{*} \mathcal{F}\right)-\frac{(-1)^{n}}{2(2 \pi \sqrt{-1})^{n} n !} \int_{\left(\mathbb{P}^{1}\right)^{n}} \operatorname{ch}_{0}\left(\varphi_{*} \operatorname{tr}_{n} \lambda \mathcal{F}\right) \sum_{i=1}^{n}(-1)^{i} S_{n}^{i}
\end{aligned}
$$

and

$$
\begin{aligned}
d_{\mathcal{D}} T_{2}(\mathcal{F}, \varphi, \Omega)+T_{2}(\partial \mathcal{F}, \varphi, \Omega) \\
=\frac{(-1)^{n}}{2(2 \pi \sqrt{-1})^{n} n !} \int_{\left(\mathbb{P}^{1}\right)^{n}} \operatorname{ch}_{0}\left(\varphi_{*} \operatorname{tr}_{n} \lambda \mathcal{F}\right) \sum_{i=1}^{n}(-1)^{i} S_{n}^{i} \\
\quad-\frac{1}{(2 \pi \sqrt{-1})^{\operatorname{dim}(M / N)}} \int_{M / N} \operatorname{Td}(\overline{T \varphi}) \operatorname{ch}_{n}(\mathcal{F}) .
\end{aligned}
$$

Hence if we write $T(\mathcal{F}, \varphi, \Omega)=T_{1}(\mathcal{F}, \varphi)+T_{2}(\mathcal{F}, \varphi, \Omega)$, then

$$
\begin{aligned}
& d_{\mathcal{D}} T(\mathcal{F}, \varphi, \Omega)+T(\partial \mathcal{F}, \varphi, \Omega) \\
& \quad=\operatorname{ch}_{n}\left(\varphi_{*} \mathcal{F}\right)-\frac{1}{(2 \pi \sqrt{-1})^{\operatorname{dim}(M / N)}} \int_{M / N} \operatorname{Td}(\overline{T \varphi}) \operatorname{ch}_{n}(\mathcal{F}) .
\end{aligned}
$$

Let us discuss dependence of $T(\mathcal{F}, \varphi, \Omega)$ on a Kähler form $\Omega$. For $u_{i} \in$ $\mathcal{D}_{1}(M)$, let

$$
C_{n}\left(u_{1}, \ldots, u_{n}\right)=\frac{1}{2^{n}} \sum_{\sigma \in \mathfrak{S}_{n}}(-1)^{\operatorname{sgn} \sigma} u_{\sigma(1)} \bullet\left(u_{\sigma(2)} \bullet\left(\cdots u_{\sigma(k)} \cdots\right)\right) .
$$


Then it is easy to show that

$$
C_{n}\left(u_{1}, \ldots, u_{n}\right)=\frac{(-1)^{n}}{2} \sum_{i=1}^{n}(-1)^{i} S_{n}^{i}\left(u_{1}, \ldots, u_{n}\right) .
$$

Lemma 7.2. For $u_{1} \in \mathcal{D}_{2}(M)$ and $u_{i} \in \mathcal{D}_{1}(M)$ with $2 \leq i \leq n$, let

$$
C_{n}\left(u_{1}, \ldots, u_{n}\right)=\frac{1}{2^{n}} \sum_{j=1}^{n}(-1)^{j+1} \sum_{\substack{\sigma \in \mathfrak{S}_{n} \\ \sigma(j)=1}}(-1)^{\operatorname{sgn} \sigma} u_{\sigma(1)} \bullet\left(u_{\sigma(2)} \bullet\left(\cdots u_{\sigma(j)} \cdots\right)\right) \text {. }
$$

Then we have

$$
\begin{aligned}
& d_{\mathcal{D}} C_{n}\left(u_{1}, u_{2}, \ldots, u_{n}\right) \\
& \quad=C_{n}\left(d_{\mathcal{D}} u_{1}, u_{2}, \ldots, u_{n}\right)+\frac{n}{2} \sum_{k=2}^{n}(-1)^{k}\left(d_{\mathcal{D}} u_{k}\right) \bullet C_{n-1}\left(u_{1}, u_{2}, \ldots, \widehat{u_{k}}, \cdots, u_{n}\right) .
\end{aligned}
$$

Proof. Since $d_{\mathcal{D}}(u \bullet v)=d_{\mathcal{D}} u \bullet v+(-1)^{\operatorname{deg} u} u \bullet d_{\mathcal{D}} v$, we have

$$
\begin{aligned}
& d_{\mathcal{D}} C_{n}\left(u_{1}, u_{2}, \ldots, u_{n}\right) \\
& =\frac{1}{2^{n}} \sum_{j=1}^{n} \sum_{\sigma \in \mathfrak{S}_{n}}(-1)^{\operatorname{sgn} \sigma} \sum_{i<j}(-1)^{i+j} d_{\mathcal{D}} u_{\sigma(i)}\left(u_{\sigma(1)} \bullet\left(\cdots \widehat{u_{\sigma(i)}} \cdots u_{\sigma(j)} \cdots\right)\right) \\
& \sigma(j)=1 \\
& +\frac{1}{2^{n}} \sum_{j=1}^{n} \sum_{\substack{\sigma \in \mathfrak{S}_{n} \\
\sigma(j)=1}}(-1)^{\operatorname{sgn} \sigma} u_{\sigma(1)} \bullet\left(\cdots d_{\mathcal{D}} u_{\sigma(j)} \cdots\right) \\
& +\frac{1}{2^{n}} \sum_{j=1}^{n} \sum_{\substack{\sigma \in \mathfrak{S}_{n} \\
\sigma(j)=1}}(-1)^{\operatorname{sgn} \sigma} \sum_{j<i}(-1)^{i+j+1} d_{\mathcal{D}} u_{\sigma(i)}\left(u_{\sigma(1)} \bullet\left(\cdots u_{\sigma(j)} \cdots \widehat{u_{\sigma(i)}} \cdots\right)\right) \\
& =\frac{1}{2^{n}} \sum_{j=1}^{n} \sum_{\sigma \in \mathfrak{S}_{n}}(-1)^{\operatorname{sgn} \sigma} u_{\sigma(1)} \bullet\left(\cdots d_{\mathcal{D}} u_{\sigma(j)} \cdots\right) \\
& \sigma(j)=1 \\
& +\frac{1}{2^{n}} \sum_{k=2}^{n} \sum_{\substack { i<j \\
\begin{subarray}{c}{\sigma \in \mathfrak{S}_{n} \\
\sigma(j)=1 \\
\sigma(i)=k{ i < j \\
\begin{subarray} { c } { \sigma \in \mathfrak { S } _ { n } \\
\sigma ( j ) = 1 \\
\sigma ( i ) = k } }\end{subarray}}(-1)^{\operatorname{sgn} \sigma}(-1)^{i+j} d_{\mathcal{D}} u_{k}\left(u_{\sigma(1)} \bullet\left(\cdots \widehat{u_{\sigma(i)}} \cdots u_{\sigma(j)} \cdots\right)\right) \\
& +\frac{1}{2^{n}} \sum_{k=2}^{n} \sum_{j<i} \sum_{\substack{\sigma \in \mathfrak{S}_{n} \\
\sigma(j)=1 \\
\sigma(i)=k}}(-1)^{\operatorname{sgn} \sigma}(-1)^{i+j+1} d_{\mathcal{D}} u_{k}\left(u_{\sigma(1)} \bullet\left(\cdots u_{\sigma(j)} \cdots \widehat{u_{\sigma(i)}} \cdots\right)\right)
\end{aligned}
$$




$$
=C_{n}\left(d_{\mathcal{D}} u_{1}, u_{2}, \ldots, u_{n}\right)+\frac{n}{2} \sum_{k=2}^{n}(-1)^{k}\left(d_{\mathcal{D}} u_{k}\right) C_{n-1}\left(u_{1}, \ldots, \widehat{u_{k}}, \ldots, u_{n}\right) .
$$

Proposition 7.3. Let $\Omega$ and $\Omega^{\prime}$ be Kähler forms with respect to a smooth hermitian metric $h_{\varphi}$ on $T \varphi$. For an exact hermitian $n$-cube $\mathcal{F}$ made of $\varphi$-acyclic vector bundles on $M$, let us write $\mu(\mathcal{F})$ for $\mu\left(\operatorname{tr}_{n} \lambda \mathcal{F}, \Omega, \Omega^{\prime}\right) \in$ $\mathcal{D}_{2}\left(N \times\left(\mathbb{P}^{1}\right)^{n}\right)$. Then we have

$$
\begin{aligned}
& T(\mathcal{F}, \varphi, \Omega)-T\left(\mathcal{F}, \varphi, \Omega^{\prime}\right) \\
& \quad \equiv \frac{-2}{(2 \pi \sqrt{-1})^{n-1} n !} \int_{\left(\mathbb{P}^{1}\right)^{n-1}} C_{n}\left(\mu(\partial \mathcal{F}), \log \left|z_{1}\right|^{2}, \ldots, \log \left|z_{n-1}\right|^{2}\right)
\end{aligned}
$$

modulo $\operatorname{Im} d_{\mathcal{D}}$.

Proof. It follows from the definition that

$$
\begin{aligned}
& T_{2}(\mathcal{F}, \varphi, \Omega) \\
& \quad=\frac{2}{(2 \pi \sqrt{-1})^{n}(n+1) !} \int_{\left(\mathbb{P}^{1}\right)^{n}} C_{n+1}\left(T\left(\operatorname{tr}_{n} \lambda \mathcal{F}, \varphi, \Omega\right), \log \left|z_{1}\right|^{2}, \ldots, \log \left|z_{n}\right|^{2}\right) .
\end{aligned}
$$

Then by Lemma 7.2 we have

$$
\begin{aligned}
& T(\mathcal{F}, \varphi, \Omega)-T\left(\mathcal{F}, \varphi, \Omega^{\prime}\right)=T_{2}(\mathcal{F}, \varphi, \Omega)-T_{2}\left(\mathcal{F}, \varphi, \Omega^{\prime}\right) \\
&=\frac{2}{(2 \pi \sqrt{-1})^{n}(n+1) !} \\
& \int_{\left(\mathbb{P}^{1}\right)^{n}} C_{n+1}\left(T\left(\operatorname{tr}_{n} \lambda \mathcal{F}, \varphi, \Omega\right)-T\left(\operatorname{tr}_{n} \lambda \mathcal{F}, \varphi, \Omega^{\prime}\right), \log \left|z_{1}\right|^{2}, \ldots, \log \left|z_{n}\right|^{2}\right) \\
&= \frac{2}{(2 \pi \sqrt{-1})^{n}(n+1) !} \int_{\left(\mathbb{P}^{1}\right)^{n}} C_{n+1}\left(d_{\mathcal{D}} \mu(\mathcal{F}), \log \left|z_{1}\right|^{2}, \ldots, \log \left|z_{n}\right|^{2}\right) \\
&= \frac{2}{(2 \pi \sqrt{-1})^{n}(n+1) !} \int_{\left(\mathbb{P}^{1}\right)^{n}} d_{\mathcal{D}} C_{n+1}\left(\mu(\mathcal{F}), \log \left|z_{1}\right|^{2}, \ldots, \log \left|z_{n}\right|^{2}\right) \\
&-\frac{1}{(2 \pi \sqrt{-1})^{n} n !} \sum_{k=1}^{n}(-1)^{k-1} \\
& \int_{\left(\mathbb{P}^{1}\right)^{n}} d_{\mathcal{D}} \log \left|z_{k}\right|^{2} C_{n}\left(\mu(\mathcal{F}), \log \left|z_{1}\right|^{2}, \ldots, \log \left|z_{k}\right|^{2}, \ldots, \log \left|z_{n}\right|^{2}\right) \\
&= \frac{2}{(2 \pi \sqrt{-1})^{n}(n+1) !} d_{\mathcal{D}}\left(\int_{\left(\mathbb{P}^{1}\right)^{n}} C_{n+1}\left(\mu(\mathcal{F}), \log \left|z_{1}\right|^{2}, \ldots, \log \left|z_{n}\right|^{2}\right)\right) \\
&-\frac{2}{(2 \pi \sqrt{-1})^{n-1} n !} \int_{\left(\mathbb{P}^{1}\right)^{n-1}} C_{n}\left(\mu(\partial \mathcal{F}), \log \left|z_{1}\right|^{2}, \ldots, \log \left|z_{n-1}\right|^{2}\right) .
\end{aligned}
$$




\section{§7.3. Definition of direct image homomorphism}

In this subsection, we apply the results obtained so far to an arithmetic situation and define a direct image homomorphism in higher arithmetic $K$ theory. Let $\varphi: X \rightarrow Y$ be a smooth projective morphism of proper arithmetic varieties. We fix an $F_{\infty}$-invariant smooth hermitian metric $h_{\varphi}$ on $T \varphi(\mathbb{C})$ such that $\left(\varphi(\mathbb{C}), h_{\varphi}\right)$ is a Kähler fibration, and take an anti- $F_{\infty}$-invariant Kähler form $\Omega$ on $X(\mathbb{C})$ with respect to $h_{\varphi}$. Let $\widehat{S}(\varphi$-ac) denote the S-construction of the category of $\varphi$-acyclic hermitian vector bundles on $X$. Then the natural inclusion $\widehat{S}(\varphi-\mathrm{ac}) \rightarrow \widehat{S}(X)$ is a homotopy equivalence, and the direct image of a $\varphi$-acyclic hermitian vector bundle with the $L_{2}$-metric gives a map of simplicial sets $\varphi_{*}: \widehat{S}(\varphi$-ac $) \rightarrow \widehat{S}(Y)$.

Proposition 7.4. If $E$ is a degenerate element of $\widehat{S}_{n+1}(\varphi-a c)$, then $T(\operatorname{Cub}(E), \varphi, \Omega)=0$.

The proof is similar to that of Theorem 4.4, so we omit it. By virtue of this proposition, taking higher analytic torsion forms yields a homomorphism

$$
T(\varphi, \Omega): C_{*}(|\widehat{S}(\varphi-\mathrm{ac})|) \rightarrow \mathcal{D}_{*}(Y) .
$$

In particular, the higher analytic torsion form of a pointed cellular map $f$ : $S^{n+1} \rightarrow \mid \widehat{S}(\varphi$-ac $) \mid$ is defined by $T(f, \varphi, \Omega)=T\left(f_{*}\left(\left[S^{n+1}\right]\right), \varphi, \Omega\right)$. We abbreviate $T(f, \varphi, \Omega)$ to $T(f)$ if the morphism $\varphi$ and the Kähler form $\Omega$ are fixed.

Let $\varphi_{!}: \mathcal{D}_{*}(X) \rightarrow \mathcal{D}_{*}(Y)$ be the map given by

$$
\varphi ! \omega=\frac{1}{(2 \pi \sqrt{-1})^{\operatorname{dim}(X / Y)}} \int_{X(\mathbb{C}) / Y(\mathbb{C})} \operatorname{Td}(\overline{T \varphi}) \omega .
$$

Then by Theorem 7.1 the diagram

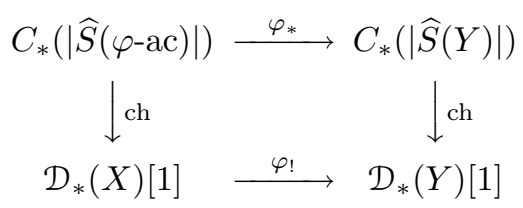

is commutative up to the homotopy $-T(, \varphi, \Omega)$. Hence by Proposition 3.9 we can obtain a homomorphism

$$
\widehat{\varphi}(\Omega)_{*}: \widehat{\pi}_{n+1}(\mid \widehat{S}(\varphi \text {-ac }) \mid, \mathrm{ch}) \rightarrow \widehat{\pi}_{n+1}(|\widehat{S}(Y)|, \mathrm{ch})
$$

by $[(f, \omega)] \mapsto\left[\left(\varphi_{*} f, \varphi ! \omega+T(f, \varphi, \Omega)\right)\right]$. 
If $\Omega^{\prime}$ is another anti- $F_{\infty}$-invariant Kähler form with respect to $h_{\varphi}$, then it follows from Proposition 7.3 that $T(f, \varphi, \Omega) \equiv T\left(f, \varphi, \Omega^{\prime}\right)$ modulo $\operatorname{Im} d_{\mathcal{D}}$ for any pointed cellular map $f: S^{n+1} \rightarrow \mid \widehat{S}(\varphi$-ac $) \mid$. Hence the homomorphism $\widehat{\varphi}(\Omega)_{*}$ depends only on the hermitian metric $h_{\varphi}$ and does not concern the Kähler form $\Omega$.

Summing up the arguments in this subsection leads to the following:

Theorem 7.5. Let $\varphi: X \rightarrow Y$ be a smooth projective morphism of proper arithmetic varieties. We fix an $F_{\infty}$-invariant metric $h_{\varphi}$ on $T \varphi$ such that $\left(\varphi(\mathbb{C}), h_{\varphi}\right)$ is a Kähler fibration. Then we can define a direct image homomorphism $\widehat{\varphi}\left(h_{\varphi}\right)_{*}: \widehat{K}_{n}(X) \rightarrow \widehat{K}_{n}(Y)$ by

$$
\widehat{\pi}_{n+1}(|\widehat{S}(X)|, \mathrm{ch}) \simeq \widehat{\pi}_{n+1}(|\widehat{S}(\varphi-a c)|, \mathrm{ch}) \stackrel{\widehat{\varphi}(\Omega)_{*}}{\longrightarrow} \widehat{\pi}_{n+1}(|\widehat{S}(Y)|, \mathrm{ch}),
$$

where $\Omega$ is an anti- $F_{\infty}$-invariant Kähler from on $X(\mathbb{C})$ with respect to $h_{\varphi}$.

When $n=0$, the isomorphism $\widehat{\alpha}: \widehat{\mathcal{K}}_{0}(X) \rightarrow \widehat{K}_{0}(X)$ gives an identification between the direct image homomorphism defined above and $\varphi_{!}: \widehat{\mathcal{K}}_{0}(X) \rightarrow$ $\widehat{\mathcal{K}}_{0}(Y)$ in [10].

Proposition 3.10 implies that the diagram

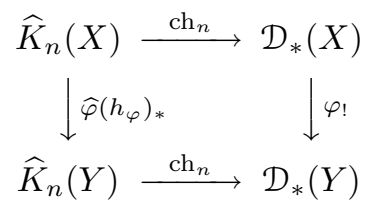

is commutative. In particular, we can obtain a direct image homomorphism in $K M$-groups

$$
\widehat{\varphi}\left(h_{\varphi}\right)_{*}: K M_{n}(X) \rightarrow K M_{n}(Y) .
$$

Finally, we give a description of the direct image homomorphism by means of the $G$-construction. Given a pointed cellular map $f: S^{n} \rightarrow|\widehat{G}(X)|$ for $n \geq 1$, let $T(f, \varphi, \Omega)=T\left(\chi_{*} f_{*}\left(\left[S^{n}\right]\right), \varphi, \Omega\right)$. Then we can obtain a homomorphism $\widehat{\varphi}(\Omega)_{*}: \widehat{\pi}_{n}(|\widehat{G}(\varphi-\mathrm{ac})|, \mathrm{ch}) \rightarrow \widehat{\pi}_{n}(|\widehat{G}(Y)|, \mathrm{ch})$ by

$$
\widehat{\varphi}(\Omega)_{*}([(f, \omega)])=\left[\left(\varphi_{*} f, \varphi_{!} \omega-T(f, \varphi, \Omega)\right)\right],
$$

and it satisfies the following commutative diagram:

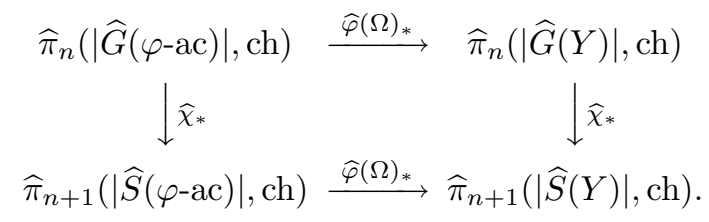


Hence the direct image homomorphism in $\widehat{K}_{*}(X)$ can also be given as follows:

$$
\widehat{\pi}_{n}(|\widehat{G}(X)|, \mathrm{ch}) \simeq \widehat{\pi}_{n}(|\widehat{G}(\varphi-\mathrm{ac})|, \mathrm{ch}) \stackrel{\widehat{\varphi}(\Omega)_{*}}{\longrightarrow} \widehat{\pi}_{n}(|\widehat{G}(Y)|, \mathrm{ch}) .
$$

\section{$\S 7.4$. The projection formula}

In this subsection we prove the projection formula in higher arithmetic $K$-theory. We first consider the case of $\widehat{\mathcal{K}}_{0}$-groups. Let $\varphi: X \rightarrow Y, h_{\varphi}$ and $\Omega$ be as in the last subsection. Let $\bar{E}$ be a hermitian vector bundle on $Y$ and $\bar{F}$ a $\varphi$-acyclic hermitian vector bundle on $X$. Then the canonical isomorphism $\varphi_{*}\left(\varphi^{*} \bar{E} \otimes \bar{F}\right) \simeq \bar{E} \otimes \varphi_{*} \bar{F}$ preserves the metrics.

Let $\varphi$ ! denote the direct image homomorphism in $\widehat{\mathcal{K}}_{0}$ given in [10]. For $\omega \in \widetilde{\mathcal{D}}_{1}(Y)$ and $\tau \in \widetilde{\mathcal{D}}_{1}(X)$, we have

$$
\begin{aligned}
\varphi_{!}\left(\widehat{\varphi}^{*}(\bar{E}, \omega) \times(\bar{F}, \tau)\right) & \\
= & \varphi_{!}\left(\left(\varphi^{*} \bar{E}\right) \otimes \bar{F}, \varphi^{*} \omega \wedge \operatorname{ch}_{0}(\bar{F})+\varphi^{*} \operatorname{ch}_{0}(\bar{E}) \wedge \tau+\varphi^{*} d_{\mathcal{D}} \omega \wedge \tau\right) \\
= & \left(\varphi_{*}\left(\varphi^{*} \bar{E} \otimes \bar{F}\right), \eta\right),
\end{aligned}
$$

where

$$
\begin{aligned}
\eta= & \frac{1}{(2 \pi \sqrt{-1})^{\operatorname{dim}(X / Y)}} \int_{X(\mathbb{C}) / Y(\mathbb{C})} \operatorname{Td}(\overline{T \varphi}) \wedge\left(\varphi^{*} \omega \wedge \operatorname{ch}_{0}(\bar{F})\right. \\
& \left.+\varphi^{*} \operatorname{ch}_{0}(\bar{E}) \wedge \tau+\varphi^{*} d_{\mathcal{D}} \omega \wedge \tau\right) \\
& -T\left(\varphi^{*} \bar{E} \otimes \bar{F}\right) \\
= & \frac{1}{(2 \pi \sqrt{-1})^{\operatorname{dim}(X / Y)}}\left(\operatorname{ch}_{0}(\bar{E})+d_{\mathcal{D}} \omega\right) \wedge \int_{X(\mathbb{C}) / Y(\mathbb{C})} \operatorname{Td}(\overline{T \varphi}) \wedge \tau \\
& +\omega \wedge\left(\operatorname{ch}_{0}\left(\varphi_{*} \bar{F}\right)-d_{\mathcal{D}} T(\bar{F})\right)-T\left(\varphi^{*} \bar{E} \otimes \bar{F}\right) .
\end{aligned}
$$

On the other hand, we have

$$
\begin{aligned}
(\bar{E}, \omega) \times \varphi_{!}(\bar{F}, \tau) & =(\bar{E}, \omega) \otimes\left(\varphi_{*} \bar{F}, \varphi_{!} \tau-T(\bar{F})\right) \\
& =\left(\bar{E} \otimes \varphi_{*} \bar{F}, \eta^{\prime}\right),
\end{aligned}
$$

where

$$
\begin{aligned}
\eta^{\prime}= & \frac{1}{(2 \pi \sqrt{-1})^{\operatorname{dim}(X / Y)}}\left(\operatorname{ch}_{0}(\bar{E})+d_{\mathcal{D}} \omega\right) \wedge \int_{X(\mathbb{C}) / Y(\mathbb{C})} \operatorname{Td}(\overline{T \varphi}) \wedge \tau \\
& +\omega \wedge \operatorname{ch}_{0}\left(\varphi_{*} \bar{F}\right)-\left(\operatorname{ch}_{0}(\bar{E})+d_{\mathcal{D}} \omega\right) \wedge T(\bar{F}) .
\end{aligned}
$$

Comparing these identities, we have

$$
\eta-\eta^{\prime}=-T\left(\varphi^{*} \bar{E} \otimes \bar{F}\right)+\operatorname{ch}_{0}(\bar{E}) \wedge T(\bar{F})+d_{\mathcal{D}}(\omega \wedge T(\bar{F})) .
$$


Hence the projection formula in $\widehat{\mathcal{K}}_{0}$-groups is reduced to the following proposition:

Proposition 7.6. Under the above notations, we have

$$
T\left(\varphi^{*} \bar{E} \otimes \bar{F}\right)=\operatorname{ch}_{0}(\bar{E}) \wedge T(\bar{F}) .
$$

Proof. Let $\mathcal{E}$ be the infinite dimensional vector bundle on $N$ consisting of smooth sections of $\Lambda^{*} T^{*(1,0)} \varphi \otimes \bar{F}$. Let $B_{u}$ and $N_{u}$ denote the Bismut superconnection and the number operator on $\mathcal{E}$ respectively. Let $\mathcal{E}^{\prime}$ be the infinite dimensional vector bundle consisting of smooth sections of $\Lambda^{*} T^{*(1,0)} \varphi \otimes$ $\left(\varphi^{*} \bar{E} \otimes \bar{F}\right)$. Let $B_{u}^{\prime}$ and $N_{u}^{\prime}$ denote the Bismut superconnection and the number operator on $\mathcal{E}^{\prime}$ respectively. Then we have a canonical isometry $\mathcal{E}^{\prime} \simeq \bar{E} \otimes \mathcal{E}$ and under this identification, we have $B_{u}^{\prime}=1 \otimes B_{u}+\nabla_{\bar{E}} \otimes 1$ and $N_{u}^{\prime}=1 \otimes N_{u}$. Substituting these into the definition of $T\left(\varphi^{*} \bar{E} \otimes \bar{F}\right)$ in [2] yields the desired identity.

Let us move on to the higher case. We assume that $n, m \geq 1$. Consider the following diagram:

$$
\begin{aligned}
& \widehat{G}(Y) \wedge \widehat{G}(\varphi-\mathrm{ac}) \stackrel{m^{G}\left(\varphi^{*} \wedge 1\right)}{\longrightarrow} \widehat{G}^{(2)}(\varphi-\mathrm{ac}) \\
& \downarrow 1 \wedge \varphi_{*} \quad \downarrow \varphi_{*} \\
& \widehat{G}(Y) \wedge \widehat{G}(Y) \stackrel{m^{G}}{\longrightarrow} \widehat{G}^{(2)}(Y) .
\end{aligned}
$$

This diagram is commutative up to a homotopy arising from the isometry $\varphi_{*}\left(\varphi^{*} \bar{E} \otimes \bar{F}\right) \simeq \bar{E} \otimes \varphi_{*} \bar{F}$. Hence for two pointed cellular maps $f: S^{n} \rightarrow|\widehat{G}(Y)|$ and $g: S^{m} \rightarrow \mid \widehat{G}(\varphi$-ac $) \mid,\left[\left(\varphi_{*}\left(\varphi^{*} f \times g\right), 0\right)\right]=\left[\left(f \times \varphi_{*} g, 0\right)\right]$. For $\omega \in \widetilde{\mathcal{D}}_{n+1}(Y)$ and $\tau \in \widetilde{\mathcal{D}}_{m+1}(X)$,

$$
\begin{aligned}
\varphi(\Omega)_{*}\left(\varphi^{*}(f, \omega) \times(g, \tau)\right)= & \varphi(\Omega)_{*}\left(\varphi^{*} f \times g,(-1)^{n} \varphi^{*} \operatorname{ch}_{n}(f) \bullet \tau+\varphi^{*} \omega \bullet \operatorname{ch}_{m}(g)\right. \\
& \left.+(-1)^{n} d_{\mathcal{D}} \varphi^{*} \omega \bullet \tau+(-1)^{n} \varphi^{*} \operatorname{ch}_{n}(f) \triangle \operatorname{ch}_{m}(g)\right) \\
= & \left(\varphi_{*}\left(\varphi^{*} f \times g\right), \eta\right),
\end{aligned}
$$

where

$$
\begin{aligned}
\eta= & \frac{(-1)^{n}}{(2 \pi \sqrt{-1})^{\operatorname{dim}(X / Y)}}\left(\operatorname{ch}_{n}(f)+d_{\mathcal{D}} \omega\right) \bullet \int_{X(\mathbb{C}) / Y(\mathbb{C})} \operatorname{Td}(\overline{T \varphi}) \wedge \tau \\
& +\omega \bullet\left(\operatorname{ch}_{m}\left(\varphi_{*} g\right)-d_{\mathcal{D}} T(g)\right) \\
& +(-1)^{n} \operatorname{ch}_{n}(f) \Delta\left(\operatorname{ch}_{m}\left(\varphi_{*} g\right)-d_{\mathcal{D}} T(g)\right)-T\left(\varphi^{*} f \times g\right) .
\end{aligned}
$$


On the other hand, we have

$$
\begin{aligned}
(f, \omega) \times \varphi\left(h_{\varphi}\right)_{*}(g, \tau) & =(f, \omega) \times\left(\varphi_{*} g, \varphi_{!} \tau-T(g)\right) \\
& =\left(f \times \varphi_{*} g, \eta^{\prime}\right),
\end{aligned}
$$

where

$$
\begin{aligned}
\eta^{\prime}= & \frac{(-1)^{n}}{(2 \pi \sqrt{-1})^{\operatorname{dim}(X / Y)}}\left(\operatorname{ch}_{n}(f)+d_{\mathcal{D}} \omega\right) \bullet \int_{X(\mathbb{C}) / Y(\mathbb{C})} \operatorname{Td}(\overline{T \varphi}) \wedge \tau \\
& -(-1)^{n}\left(\operatorname{ch}_{n}(f)+d_{\mathcal{D}} \omega\right) \bullet T(g) \\
& +\omega \bullet \operatorname{ch}_{n}\left(\varphi_{*} g\right)+(-1)^{n} \operatorname{ch}_{n}(f) \triangle \operatorname{ch}_{m}\left(\varphi_{*} g\right) .
\end{aligned}
$$

Hence we have

$$
\eta-\eta^{\prime} \equiv(-1)^{n+1} \operatorname{ch}_{n}(f) \triangle d_{\mathcal{D}} T(g)-T\left(\varphi^{*} f \times g\right)+(-1)^{n} \operatorname{ch}_{n}(f) \bullet T(g)
$$

modulo $\operatorname{Im} d_{\mathcal{D}}$. Thus the projection formula in higher arithmetic $K$-theory is reduced to the following proposition:

Proposition 7.7. $\quad$ For an exact hermitian $n$-cube $\mathcal{F}$ on $Y$ and an exact hermitian $m$-cube $\mathcal{G}$ made of $\varphi$-acyclic vector bundles on $X$, we have

$$
\begin{aligned}
d_{\mathcal{D}}\left(\operatorname{ch}_{n}(\mathcal{F}) \Delta T(\mathcal{G})\right)= & -T\left(\varphi^{*} \mathcal{F} \otimes \mathcal{G}\right)+(-1)^{n} \operatorname{ch}_{n}(\mathcal{F}) \bullet T(\mathcal{G}) \\
& +\operatorname{ch}_{n+1}(\partial \mathcal{F}) \triangle T(\mathcal{G})+(-1)^{n-1} \operatorname{ch}_{n}(\mathcal{F}) \triangle d_{\mathcal{D}} T(\mathcal{G})
\end{aligned}
$$

Proof. We will prove the following identities:

$$
\begin{aligned}
d_{\mathcal{D}}\left(\operatorname{ch}_{n}(\mathcal{F}) \triangle T_{1}(\mathcal{G})\right)= & -T_{1}\left(\varphi^{*} \mathcal{F} \times \mathcal{G}\right)+(-1)^{n} \operatorname{ch}_{n}(\mathcal{F}) \bullet T_{1}(\mathcal{G}) \\
& +\operatorname{ch}_{n-1}(\partial \mathcal{F}) \Delta T_{1}(\mathcal{G})+(-1)^{n+1} \operatorname{ch}_{n}(\mathcal{F}) \Delta d_{\mathcal{D}} T_{1}(\mathcal{G}), \\
d_{\mathcal{D}}\left(\operatorname{ch}_{n}(\mathcal{F}) \triangle T_{2}(\mathcal{G})\right)= & -T_{2}\left(\varphi^{*} \mathcal{F} \times \mathcal{G}\right)+(-1)^{n} \operatorname{ch}_{n}(\mathcal{F}) \bullet T_{2}(\mathcal{G}) \\
& +\operatorname{ch}_{n-1}(\partial \mathcal{F}) \Delta T_{2}(\mathcal{G})+(-1)^{n+1} \operatorname{ch}_{n}(\mathcal{F}) \triangle d_{\mathcal{D}} T_{2}(\mathcal{G}) .
\end{aligned}
$$

These identities can be proved in the same way, so we will prove only the latter one.

For $t<s$, let $\pi_{1}:\left(\mathbb{P}^{1}\right)^{s} \rightarrow\left(\mathbb{P}^{1}\right)^{t}$ denote the projection $\pi_{1}\left(x_{1}, \ldots, x_{s}\right)=$ $\left(x_{1}, \ldots, x_{t}\right)$ and $\pi_{2}:\left(\mathbb{P}^{1}\right)^{s} \rightarrow\left(\mathbb{P}^{1}\right)^{t}$ denote the projection $\pi_{2}\left(x_{1}, \ldots, x_{s}\right)=$ $\left(x_{s-t+1}, \ldots, x_{s}\right)$. Then Proposition 5.5 implies that

$$
\begin{aligned}
& d\left(\operatorname{ch}_{n}(\mathcal{F}) \triangle T_{2}(\mathcal{G})\right) \\
& =\frac{(-1)^{n+m+1}}{(2 \pi \sqrt{-1})^{n+m-1} n !(m+1) !} \int_{\left(\mathbb{P}^{1}\right)^{n+m}} \pi_{1}^{*} \operatorname{ch}_{0}\left(\operatorname{tr}_{n} \lambda \mathcal{F}\right)
\end{aligned}
$$




$$
\begin{aligned}
& \wedge d\left(\sum_{\substack{1 \leq i \leq n \\
1 \leq j \leq m+1}}(-1)^{i+j} \pi_{1}^{*} S_{n}^{i} \Delta \pi_{2}^{*} S_{m+1}^{j}(\mathcal{G})\right) \\
& =\frac{(-1)^{n+m+1}}{(2 \pi \sqrt{-1})^{n+m}(n+m+1) !} \int_{\left(\mathbb{P}^{1}\right)^{n+m}} \pi_{1}^{*} \operatorname{ch}_{0}\left(\operatorname{tr}_{n} \lambda \mathcal{F}\right) \sum_{k=1}^{n+m+1}(-1)^{k} \\
& S_{n+m+1}^{k}\left(\pi_{2}^{*} T\left(\operatorname{tr}_{m} \lambda \mathcal{G}\right), \log \left|t_{1}\right|^{2}, \ldots, \log \left|t_{n+m}\right|^{2}\right) \\
& +\frac{(-1)^{n+m+1}}{2(2 \pi \sqrt{-1})^{n+m-1}(n-1) !(m+1) !} \int_{\left(\mathbb{P}^{1}\right)^{n+m-1}} \pi_{1}^{*} \operatorname{ch}_{0}\left(\operatorname{tr}_{n-1} \lambda \partial \mathcal{F}\right) \\
& \wedge \sum_{\substack{1 \leq i \leq n-1 \\
1 \leq j \leq m+1}}(-1)^{i+j} a_{i, j}^{n-1, m+1} \pi_{1}^{*} S_{n-1}^{i} \wedge \pi_{2}^{*} S_{m+1}^{j}(\mathcal{G}) \\
& +\frac{(-1)^{m+1}}{2(2 \pi \sqrt{-1})^{n+m} n ! m !} \int_{\left(\mathbb{P}^{1}\right)^{n+m}} \pi_{1}^{*} \operatorname{ch}_{0}\left(\operatorname{tr}_{n} \lambda \mathcal{F}\right) \\
& \wedge \sum_{\substack{1 \leq i \leq n \\
1 \leq j \leq m}}(-1)^{i+j} a_{i, j}^{n, m} \pi_{1}^{*} S_{n}^{i} \wedge \pi_{2}^{*}\left(\partial \bar{\partial} T\left(\operatorname{tr}_{m} \lambda \mathcal{G}\right) \wedge S_{m}^{j}\right) \\
& +\frac{(-1)^{m+1}}{2(2 \pi \sqrt{-1})^{n+m-1} n ! m !} \int_{\left(\mathbb{P}^{1}\right)^{n+m-1}} \pi_{1}^{*} \operatorname{ch}_{0}\left(\operatorname{tr}_{n} \lambda \mathcal{F}\right) \\
& \wedge \sum_{\substack{1 \leq i \leq n \\
1 \leq j \leq m}}(-1)^{i+j} a_{i, j}^{n, m} \pi_{1}^{*} S_{n}^{i} \wedge \pi_{2}^{*} S_{m}^{j}(\partial \mathcal{G}) \\
& +\frac{(-1)^{m}}{2(2 \pi \sqrt{-1})^{n+m} n !(m+1) !} \int_{\left(\mathbb{P}^{1}\right)^{n+m}} \pi_{1}^{*} \operatorname{ch}_{0}\left(\operatorname{tr}_{n} \lambda \mathcal{F}\right) \\
& \wedge \sum_{\substack{1 \leq i \leq n \\
1 \leq j \leq m+1}}(-1)^{i+j} \pi_{1}^{*} S_{n}^{i} \bullet \pi_{2}^{*} S_{m+1}^{j}(\mathcal{G}) .
\end{aligned}
$$

By Proposition 7.6, we have

$$
\begin{aligned}
\pi_{1}^{*} & \operatorname{ch}_{0}\left(\operatorname{tr}_{n} \lambda \mathcal{F}\right) S_{n+m+1}^{k}\left(\pi_{2}^{*} T\left(\operatorname{tr}_{m} \lambda \mathcal{G}\right), \log \left|t_{1}\right|^{2}, \ldots, \log \left|t_{n+m}\right|^{2}\right) \\
& =S_{n+m+1}^{k}\left(\pi_{1}^{*} \operatorname{ch}_{0}\left(\operatorname{tr}_{n} \lambda \mathcal{F}\right) \wedge \pi_{2}^{*} T\left(\operatorname{tr}_{m} \lambda \mathcal{G}\right), \log \left|t_{1}\right|^{2}, \ldots, \log \left|t_{n+m}\right|^{2}\right) \\
& =S_{n+m+1}^{k}\left(T\left(\operatorname{tr}_{n+m} \lambda\left(\varphi^{*} \mathcal{F} \otimes \mathcal{G}\right), \log \left|t_{1}\right|^{2}, \ldots, \log \left|t_{n+m}\right|^{2}\right)\right. \\
& =S_{n+m+1}^{k}\left(\varphi^{*} \mathcal{F} \otimes \mathcal{G}\right) .
\end{aligned}
$$

Moreover,

$$
d_{\mathcal{D}} T_{2}(\mathcal{G})=\frac{(-1)^{m+1}}{(2 \pi \sqrt{-1})^{m}(m+1) !} \int_{\left(\mathbb{P}^{1}\right)^{m}} \sum_{j=1}^{m}(-1)^{j+1}\left(\partial S_{m+1}^{j}(\mathcal{G})-\bar{\partial} S_{m+1}^{j+1}(\mathcal{G})\right)
$$




$$
\begin{aligned}
= & \frac{(-1)^{m+1}}{(2 \pi \sqrt{-1})^{m} m !} \int_{\left(\mathbb{P}^{1}\right)^{m}} \partial \bar{\partial} T\left(\operatorname{tr}_{m} \lambda \mathcal{G}\right) \wedge\left(\sum_{j=1}^{m}(-1)^{j} S_{m}^{j}\right) \\
& +\frac{(-1)^{m+1}}{(2 \pi \sqrt{-1})^{m-1} m !} \int_{\left(\mathbb{P}^{1}\right)^{m-1}} \sum_{j=1}^{m}(-1)^{j} S_{m}^{j}(\partial \mathcal{G}) .
\end{aligned}
$$

Hence

$$
\begin{aligned}
& d_{\mathcal{D}}\left(\operatorname{ch}_{n}(\mathcal{F}) \triangle T_{2}(\mathcal{G})\right)=-d\left(\operatorname{ch}_{n}(\mathcal{F}) \triangle T_{2}(\mathcal{G})\right) \\
& =\frac{(-1)^{n+m}}{(2 \pi \sqrt{-1})^{n+m}(n+m+1) !} \int_{\left(\mathbb{P}^{1}\right)^{n+m}} \sum_{k=1}^{n+m+1}(-1)^{k} S_{n+m+1}^{k}\left(\varphi^{*} \mathcal{F} \otimes \mathcal{G}\right) \\
& +\frac{(-1)^{n+m}}{2(2 \pi \sqrt{-1})^{n+m-1}(n-1) !(m+1) !} \\
& \int_{\left(\mathbb{P}^{1}\right)^{n+m-1}} \sum_{\substack{1 \leq i \leq n-1 \\
1 \leq j \leq m+1}}(-1)^{i+j} a_{i, j}^{n-1, m+1} \pi_{1}^{*}\left(\operatorname{ch}_{0}\left(\operatorname{tr}_{n-1} \lambda \partial \mathcal{F}\right) S_{n-1}^{i}\right) \wedge \pi_{2}^{*} S_{m+1}^{j}(\mathcal{G}) \\
& -\frac{(-1)^{m}}{2(2 \pi \sqrt{-1})^{n} n !} \sum_{\substack{1 \leq i \leq n \\
1 \leq j \leq m}}(-1)^{i+j} a_{i, j}^{n, m}\left(\int_{\left(\mathbb{P}^{1}\right)^{n}} \operatorname{ch}_{0}\left(\operatorname{tr}_{n} \lambda \mathcal{F}\right) S_{n}^{i}\right) \\
& \wedge\left(-\frac{1}{(2 \pi \sqrt{-1})^{m} m !} \int_{\left(\mathbb{P}^{1}\right)^{m}} \partial \bar{\partial} T\left(\operatorname{tr}_{m} \lambda \mathcal{G}\right) S_{m}^{j}-\frac{1}{(2 \pi \sqrt{-1})^{m-1} m !} \int_{\left(\mathbb{P}^{1}\right)^{m-1}} S_{m}^{j}(\partial \mathcal{G})\right) \\
& +\frac{(-1)^{m+1}}{2(2 \pi \sqrt{-1})^{n+m} n !(m+1) !} \\
& \int_{\left(\mathbb{P}^{1}\right)^{n+m}} \sum_{\substack{1 \leq i \leq n \\
1 \leq j \leq m+1}}(-1)^{i+j} \pi_{1}^{*}\left(\operatorname{ch}_{0}\left(\operatorname{tr}_{n} \lambda \mathcal{F}\right) S_{n}^{i}\right) \bullet \pi_{2}^{*} S_{m+1}^{j}(\mathcal{G}) \\
& =-T_{2}\left(\varphi^{*} \mathcal{F} \otimes \mathcal{G}\right)+\operatorname{ch}_{n-1}(\partial \mathcal{F}) \Delta T_{2}(\mathcal{G}) \\
& +(-1)^{n+1} \operatorname{ch}_{n}(\mathcal{F}) \Delta d_{\mathcal{D}} T_{2}(\mathcal{G})+(-1)^{n} \operatorname{ch}_{n}(\mathcal{F}) \bullet T_{2}(\mathcal{G}) \text {. }
\end{aligned}
$$

Let us consider the case of $n=0$ and $m>0$. Let $(\bar{E}, \omega)$ be a pair of a hermitian vector bundle $\bar{E}$ on $Y$ and $\omega \in \widetilde{\mathcal{D}}_{1}(Y)$ and let $(g, \tau)$ be a pair of a pointed cellular map $g: S^{m} \rightarrow \mid \widehat{G}(\varphi$-ac $) \mid$ and $\tau \in \widetilde{\mathcal{D}}_{m+1}(X)$. Then we have

$$
\begin{aligned}
\widehat{\varphi}(\Omega)_{*}( & \left.\widehat{\varphi}^{*}(\bar{E}, \omega) \times(g, \tau)\right) \\
= & \left(\varphi_{*}\left(\varphi^{*} \bar{E} \otimes g\right), \varphi_{!}\left(\varphi^{*} \omega \bullet\left(\operatorname{ch}_{m}(g)+d_{\mathcal{D}} \tau\right)\right)+\varphi_{!}\left(\varphi^{*} \operatorname{ch}_{0}(\bar{E}) \bullet \tau\right)\right. \\
& -T\left(\varphi^{*} \bar{E} \otimes g\right) \\
= & \left(\varphi_{*}\left(\varphi^{*} \bar{E} \otimes g\right), \omega \bullet \varphi_{!}\left(\operatorname{ch}_{m}(g)+d_{\mathcal{D}} \tau\right)\right)+\operatorname{ch}_{0}(\bar{E}) \bullet\left(\varphi_{!} \tau-T(g)\right) .
\end{aligned}
$$


On the other hand, we have

$$
\begin{aligned}
(\bar{E}, \omega) & \times \widehat{\varphi}(\Omega)_{*}(g, \tau) \\
& =\left(\bar{E} \otimes \varphi_{*} g, \operatorname{ch}_{0}(\bar{E}) \bullet\left(\varphi_{!} \tau-T(g)\right)+\omega \bullet \operatorname{ch}_{m}\left(\varphi_{*} g\right)+\omega \bullet\left(\varphi_{!} \tau-T(g)\right)\right. \\
& =\left(\bar{E} \otimes \varphi_{*} g, \operatorname{ch}_{0}(\bar{E}) \bullet\left(\varphi_{!} \tau-T(g)\right)+\omega \bullet d_{\mathcal{D}} \varphi_{!} \tau+\omega \bullet \varphi_{!} \operatorname{ch}_{m}(g)\right) .
\end{aligned}
$$

Hence we have

$$
\widehat{\varphi}(\Omega)_{*}\left(\widehat{\varphi}^{*}([(\bar{E}, \omega)]) \times[(g, \tau)]\right)=[(\bar{E}, \omega)] \times \widehat{\varphi}(\Omega)_{*}([(g, \tau)]) .
$$

In the case of $n>0$ and $m=0$, we can prove the projection formula for the pairing $\widehat{K}_{n} \times \widehat{\mathcal{K}}_{0} \rightarrow \widehat{K}_{n}$ in the same way. Hence we have the following theorem:

Theorem 7.8. Let $\varphi: X \rightarrow Y$ be a projective smooth morphism of proper arithmetic varieties. Let $h_{\varphi}$ be an $F_{\infty}$-invariant smooth hermitian metric on $T \varphi(\mathbb{C})$ such that $\left(\varphi(\mathbb{C}), h_{\varphi}\right)$ is a Kähler fibration. Then for $y \in \widehat{K}_{n}(Y)$ and $x \in \widehat{K}_{m}(X)$,

$$
\widehat{\varphi}\left(h_{\varphi}\right)_{*}\left(\widehat{\varphi}^{*} y \times x\right)=y \times \widehat{\varphi}\left(h_{\varphi}\right)_{*}(x) .
$$

\section{Appendix A. Some Identities Satisfied by Binomial Coefficients}

Lemma A.1. (1) For $0 \leq k \leq i$, we have

$$
\begin{aligned}
(n-i) & \sum_{\alpha=0}^{k-1}\left(\begin{array}{c}
n+m-i-j+1 \\
n-\alpha
\end{array}\right)\left(\begin{array}{c}
i+j-1 \\
\alpha
\end{array}\right)+i \sum_{\alpha=0}^{k}\left(\begin{array}{c}
n+m-i-j \\
n-\alpha
\end{array}\right)\left(\begin{array}{c}
i+j \\
\alpha
\end{array}\right) \\
& =(n+m) \sum_{\alpha=0}^{k-1}\left(\begin{array}{c}
n+m-i-j \\
n-1-\alpha
\end{array}\right)\left(\begin{array}{c}
i+j-1 \\
\alpha
\end{array}\right)+(i-k)\left(\begin{array}{c}
n+m-i-j \\
n-k
\end{array}\right)\left(\begin{array}{c}
i+j-1 \\
k
\end{array}\right) .
\end{aligned}
$$

In particular, we have

$$
\begin{gathered}
(n-i) \sum_{\alpha=0}^{i-1}\left(\begin{array}{c}
n+m-i-j+1 \\
n-\alpha
\end{array}\right)\left(\begin{array}{c}
i+j-1 \\
\alpha
\end{array}\right)+i \sum_{\alpha=0}^{i}\left(\begin{array}{c}
n+m-i-j \\
n-\alpha
\end{array}\right)\left(\begin{array}{c}
i+j \\
\alpha
\end{array}\right) \\
=(n+m) \sum_{\alpha=0}^{i-1}\left(\begin{array}{c}
n+m-i-j \\
n-1-\alpha
\end{array}\right)\left(\begin{array}{c}
i+j-1 \\
\alpha
\end{array}\right) .
\end{gathered}
$$

(2) For $0 \leq k \leq i$, we have

$$
\begin{aligned}
(m-j) & \sum_{\alpha=k}^{i-1}\left(\begin{array}{c}
n+m-i-j+1 \\
n-\alpha
\end{array}\right)\left(\begin{array}{c}
i+j-1 \\
\alpha
\end{array}\right)+j \sum_{\alpha=k}^{i-1}\left(\begin{array}{c}
n+m-i-j \\
n-\alpha
\end{array}\right)\left(\begin{array}{c}
i+j \\
\alpha
\end{array}\right) \\
& =(n+m) \sum_{\alpha=k}^{i-1}\left(\begin{array}{c}
n+m-i-j \\
n-\alpha
\end{array}\right)\left(\begin{array}{c}
i+j-1 \\
\alpha
\end{array}\right)-(i-k)\left(\begin{array}{c}
n+m-i-j \\
n-k
\end{array}\right)\left(\begin{array}{c}
i+j-1 \\
k-1
\end{array}\right) .
\end{aligned}
$$


In particular, we have

$$
\begin{gathered}
(m-j) \sum_{\alpha=0}^{i-1}\left(\begin{array}{c}
n+m-i-j+1 \\
n-\alpha
\end{array}\right)\left(\begin{array}{c}
i+j-1 \\
\alpha
\end{array}\right)+j \sum_{\alpha=0}^{i-1}\left(\begin{array}{c}
n+m-i-j \\
n-\alpha
\end{array}\right)\left(\begin{array}{c}
i+j \\
\alpha
\end{array}\right) \\
=(n+m) \sum_{\alpha=0}^{i-1}\left(\begin{array}{c}
n+m-i-j \\
n-\alpha
\end{array}\right)\left(\begin{array}{c}
i+j-1 \\
\alpha
\end{array}\right) .
\end{gathered}
$$

Proof. We will prove them by induction on $k$. When $k=0$, the claim (1) is trivial. If the claim (1) holds for $k-1$, then

$$
\begin{aligned}
& (n-i) \sum_{\alpha=0}^{k-1}\left(\begin{array}{c}
n+m-i-j+1 \\
n-\alpha
\end{array}\right)\left(\begin{array}{c}
i+j-1 \\
\alpha
\end{array}\right)+i \sum_{\alpha=0}^{k}\left(\begin{array}{c}
n+m-i-j \\
n-\alpha
\end{array}\right)\left(\begin{array}{c}
i+j \\
\alpha
\end{array}\right) \\
& =(n+m) \sum_{\alpha=0}^{k-2}\left(\begin{array}{c}
n+m-i-j \\
n-1-\alpha
\end{array}\right)\left(\begin{array}{c}
i+j-1 \\
\alpha
\end{array}\right)+(i-k+1)\left(\begin{array}{c}
n+m-i-j \\
n-k+1
\end{array}\right)\left(\begin{array}{c}
i+j-1 \\
k-1
\end{array}\right) \\
& +(n-i)\left(\begin{array}{c}
n+m-i-j+1 \\
n-k+1
\end{array}\right)\left(\begin{array}{c}
i+j-1 \\
k-1
\end{array}\right)+i\left(\begin{array}{c}
n+m-i-j \\
n-k
\end{array}\right)\left(\begin{array}{c}
i+j \\
k
\end{array}\right) \\
& =(n+m) \sum_{\alpha=0}^{k-2}\left(\begin{array}{c}
n+m-i-j \\
n-1-\alpha
\end{array}\right)\left(\begin{array}{c}
i+j-1 \\
\alpha
\end{array}\right)+(n-k+1)\left(\begin{array}{c}
n+m-i-j \\
n-k+1
\end{array}\right)\left(\begin{array}{c}
i+j-1 \\
k-1
\end{array}\right) \\
& +n\left(\begin{array}{c}
n+m-i-j \\
n-k
\end{array}\right)\left(\begin{array}{c}
i+j-1 \\
k-1
\end{array}\right)+i\left(\begin{array}{c}
n+m-i-j \\
n-k
\end{array}\right)\left(\begin{array}{c}
i+j-1 \\
k
\end{array}\right) \\
& =(n+m) \sum_{\alpha=0}^{k-1}\left(\begin{array}{c}
n+m-i-j \\
n-1-\alpha
\end{array}\right)\left(\begin{array}{c}
i+j-1 \\
\alpha
\end{array}\right)-(i+j-k)\left(\begin{array}{c}
n+m-i-j \\
n-k
\end{array}\right)\left(\begin{array}{c}
i+j-1 \\
k-1
\end{array}\right) \\
& +i\left(\begin{array}{c}
n+m-i-j \\
n-k
\end{array}\right)\left(\begin{array}{c}
i+j-1 \\
k
\end{array}\right) \\
& =(n+m) \sum_{\alpha=0}^{k-1}\left(\begin{array}{c}
n+m-i-j \\
n-1-\alpha
\end{array}\right)\left(\begin{array}{c}
i+j-1 \\
\alpha
\end{array}\right)+(i-k)\left(\begin{array}{c}
n+m-i-j \\
n-k
\end{array}\right)\left(\begin{array}{c}
i+j-1 \\
k
\end{array}\right) .
\end{aligned}
$$

Hence the claim (1) holds for $k$.

The claim (2) for $k=i$ is trivial. If (2) holds for $k+1$, then

$$
\begin{aligned}
& (m-j) \sum_{\alpha=k}^{i-1}\left(\begin{array}{c}
n+m-i-j+1 \\
n-\alpha
\end{array}\right)\left(\begin{array}{c}
i+j-1 \\
\alpha
\end{array}\right)+j \sum_{\alpha=k}^{i-1}\left(\begin{array}{c}
n+m-i-j \\
n-\alpha
\end{array}\right)\left(\begin{array}{c}
i+j \\
\alpha
\end{array}\right) \\
& =(n+m) \sum_{\alpha=k+1}^{i-1}\left(\begin{array}{c}
n+m-i-j \\
n-\alpha
\end{array}\right)\left(\begin{array}{c}
i+j-1 \\
\alpha
\end{array}\right)-(i-k-1)\left(\begin{array}{c}
n+m-i-j \\
n-k-1
\end{array}\right)\left(\begin{array}{c}
i+j-1 \\
k
\end{array}\right) \\
& \quad+(m-j)\left(\begin{array}{c}
n+m-i-j+1 \\
n-k
\end{array}\right)\left(\begin{array}{c}
i+j-1 \\
k
\end{array}\right)+j\left(\begin{array}{c}
n+m-i-j \\
n-k
\end{array}\right)\left(\begin{array}{c}
i+j \\
k
\end{array}\right) \\
& =(n+m) \sum_{\alpha=k+1}^{i-1}\left(\begin{array}{c}
n+m-i-j \\
n-\alpha
\end{array}\right)\left(\begin{array}{c}
i+j-1 \\
\alpha
\end{array}\right)
\end{aligned}
$$




$$
\begin{aligned}
& +(m-j-i+k+1)\left(\begin{array}{c}
n+m-i-j \\
n-k-1
\end{array}\right)\left(\begin{array}{c}
i+j-1 \\
k
\end{array}\right)+m\left(\begin{array}{c}
n+m-i-j \\
n-k
\end{array}\right)\left(\begin{array}{c}
i+j-1 \\
k
\end{array}\right) \\
& +j\left(\begin{array}{c}
n+m-i-j \\
n-k
\end{array}\right)\left(\begin{array}{c}
i+j-1 \\
k-1
\end{array}\right) \\
= & (n+m) \sum_{\alpha=k+1}^{i-1}\left(\begin{array}{c}
n+m-i-j \\
n-\alpha
\end{array}\right)\left(\begin{array}{c}
i+j-1 \\
\alpha
\end{array}\right)+(n+m-k)\left(\begin{array}{c}
n+m-i-j \\
n-k
\end{array}\right)\left(\begin{array}{c}
i+j-1 \\
k
\end{array}\right) \\
& +j\left(\begin{array}{c}
n+m-i-j \\
n-k
\end{array}\right)\left(\begin{array}{c}
i+j-1 \\
k-1
\end{array}\right) \\
= & (n+m) \sum_{\alpha=k}^{i-1}\left(\begin{array}{c}
n+m-i-j \\
n-\alpha
\end{array}\right)\left(\begin{array}{c}
i+j-1 \\
\alpha
\end{array}\right)-(i-k)\left(\begin{array}{c}
n+m-i-j \\
n-k
\end{array}\right)\left(\begin{array}{c}
i+j-1 \\
k-1
\end{array}\right),
\end{aligned}
$$

hence the claim (2) holds for $k$.

Lemma A.2. We have

$$
\begin{gathered}
\left(\begin{array}{c}
n+m+l \\
n
\end{array}\right)^{-1} \sum_{i=0}^{n}\left(\begin{array}{c}
n+m-i-j \\
n-i
\end{array}\right)\left(\begin{array}{c}
i+j-1 \\
i
\end{array}\right) \sum_{\alpha=0}^{i+j-1}\left(\begin{array}{c}
n+m+l-i-j-k+1 \\
n+m-\alpha
\end{array}\right)\left(\begin{array}{c}
i+j+k-1 \\
\alpha
\end{array}\right) \\
=\sum_{\alpha=0}^{j-1}\left(\begin{array}{c}
m+l-j-k+1 \\
m-\alpha
\end{array}\right)\left(\begin{array}{c}
j+k-1 \\
\alpha
\end{array}\right) .
\end{gathered}
$$

Proof. Let $F_{n}$ denote the left hand side of the above. Then we have

$$
\begin{aligned}
F_{n}= & \left(\begin{array}{c}
n+m+l \\
n
\end{array}\right)^{-1} \\
& \times \sum_{i=1}^{n}\left(\left(1-\frac{i-1}{n}\right)\left(\begin{array}{c}
n+m-i-j+1 \\
n-i+1
\end{array}\right)\left(\begin{array}{c}
i+j-2 \\
i-1
\end{array}\right) \sum_{\alpha=0}^{i+j-2}\left(\begin{array}{c}
n+m+l-i-j-k+2 \\
n+m-\alpha
\end{array}\right)\left(\begin{array}{c}
i+j+k-2 \\
\alpha
\end{array}\right)\right. \\
& \left.+\frac{i}{n}\left(\begin{array}{c}
n+m-i-j \\
n-i
\end{array}\right)\left(\begin{array}{c}
i+j-1 \\
i
\end{array}\right) \sum_{\alpha=0}^{i+j-1}\left(\begin{array}{c}
n+m+l-i-j-k+1 \\
n+m-\alpha
\end{array}\right)\left(\begin{array}{c}
i+j+k-1 \\
\alpha
\end{array}\right)\right) \\
= & \frac{1}{n}\left(\begin{array}{c}
n+m+l \\
n
\end{array}\right)-1 \\
& \times\left(\begin{array}{c}
n+m-i-j \\
n-i
\end{array}\right)\left(\begin{array}{c}
i+j-2 \\
i-1
\end{array}\right) \\
& \left.+(i+j-1) \sum_{\alpha=0}^{n+1}\left(\begin{array}{c}
n+m+l-i-j-k+1 \\
n+m-\alpha
\end{array}\right)\left(\begin{array}{c}
i+j+k-1 \\
\alpha
\end{array}\right)\right) .
\end{aligned}
$$

By Lemma A.1, we have

$$
F_{n}=\frac{n+m+l}{n}\left(\begin{array}{c}
n+m+l \\
n
\end{array}\right)^{-1} \sum_{i=1}^{n}\left(\begin{array}{c}
n+m-i-j \\
n-i
\end{array}\right)\left(\begin{array}{c}
i+j-2 \\
i-1
\end{array}\right)
$$




$$
\begin{aligned}
& \times \sum_{\alpha=0}^{i+j-2}\left(\begin{array}{c}
n+m+l-i-j-k+1 \\
n+m-1-\alpha
\end{array}\right)\left(\begin{array}{c}
i+j+k-1 \\
\alpha
\end{array}\right) \\
= & \left(\begin{array}{c}
n+m+l-1 \\
n-1
\end{array}\right)-1 \sum_{i=0}^{n-1}\left(\begin{array}{c}
n+m-i-j-1 \\
n-i-1
\end{array}\right)\left(\begin{array}{c}
i+j-1 \\
i
\end{array}\right) \sum_{\alpha=0}^{i+j-1}\left(\begin{array}{c}
n+m+l-i-j-k \\
n+m-1-\alpha
\end{array}\right)\left(\begin{array}{c}
i+j+k \\
\alpha
\end{array}\right) \\
= & F_{n-1} .
\end{aligned}
$$

Hence $F_{n}=F_{0}$, that is,

$$
F_{n}=\sum_{\alpha=0}^{j-1}\left(\begin{array}{c}
m+l-j-k+1 \\
m-\alpha
\end{array}\right)\left(\begin{array}{c}
j+k-1 \\
\alpha
\end{array}\right) .
$$

Lemma A.3. If $0 \leq i \leq n$ and $1 \leq j \leq m$, we have

$$
\sum_{\alpha=0}^{i}\left(\begin{array}{c}
n+m-\alpha-j \\
n-\alpha
\end{array}\right)\left(\begin{array}{c}
\alpha+j-1 \\
\alpha
\end{array}\right)=\sum_{\alpha=0}^{i}\left(\begin{array}{c}
n+m-i-j \\
n-\alpha
\end{array}\right)\left(\begin{array}{c}
i+j \\
\alpha
\end{array}\right) .
$$

In particular, we have

$$
\sum_{\alpha=0}^{n}\left(\begin{array}{c}
n+m-\alpha-j \\
n-\alpha
\end{array}\right)\left(\begin{array}{c}
\alpha+j-1 \\
\alpha
\end{array}\right)=\sum_{\alpha=0}^{n}\left(\begin{array}{c}
m-j \\
n-\alpha
\end{array}\right)\left(\begin{array}{c}
n+j \\
\alpha
\end{array}\right)=\left(\begin{array}{c}
n+m \\
n
\end{array}\right) .
$$

Proof. We prove the lemma by induction on $i$. When $i=0$, the statement of the lemma is clear. If the identity holds for $i-1$, then

$$
\begin{aligned}
\sum_{\alpha=0}^{i}\left(\begin{array}{c}
n+m-\alpha-j \\
n-\alpha
\end{array}\right)\left(\begin{array}{c}
\alpha+j-1 \\
\alpha
\end{array}\right)= & \sum_{\alpha=0}^{i-1}\left(\begin{array}{c}
n+m-i-j+1 \\
n-\alpha
\end{array}\right)\left(\begin{array}{c}
i+j-1 \\
\alpha
\end{array}\right)+\left(\begin{array}{c}
n+m-i-j \\
n-i
\end{array}\right)\left(\begin{array}{c}
i+j-1 \\
i
\end{array}\right) \\
= & \sum_{\alpha=0}^{i-1}\left(\begin{array}{c}
n+m-i-j \\
n-\alpha
\end{array}\right)\left(\begin{array}{c}
i+j-1 \\
\alpha
\end{array}\right) \\
& +\sum_{\alpha=0}^{i-1}\left(\begin{array}{c}
n+m-i-j \\
n-1-\alpha
\end{array}\right)\left(\begin{array}{c}
i+j-1 \\
\alpha
\end{array}\right)+\left(\begin{array}{c}
n+m-i-j \\
n-i
\end{array}\right)\left(\begin{array}{c}
i+j-1 \\
i
\end{array}\right) \\
= & \sum_{\alpha=0}^{i}\left(\begin{array}{c}
n+m-i-j \\
n-\alpha
\end{array}\right)\left(\begin{array}{c}
i+j-1 \\
\alpha
\end{array}\right)+\sum_{\alpha=1}\left(\begin{array}{c}
n+m-i-j \\
n-\alpha
\end{array}\right)\left(\begin{array}{c}
i+j-1 \\
\alpha-1
\end{array}\right) \\
= & \sum_{\alpha=0}^{i}\left(\begin{array}{c}
n+m-i-j \\
n-\alpha
\end{array}\right)\left(\begin{array}{c}
i+j \\
\alpha
\end{array}\right) .
\end{aligned}
$$




\section{References}

[1] Beilinson, A. A., Higher regulators and values of $L$-functions, J. Soviet Math., 30 (1985), 2036-2070.

[2] Bismut, J.-M. and Köhler, K., Higher analytic torsion forms for direct images and anomary formulas, J. Algebraic Geom., 1 (1992), 647-684.

[3] Borel, A., Cohomologie de $S L_{n}$ et valeurs de fonctions zêta, Ann. Scuola Norm. Sup. Pisa Cl. Sci., 4 (1977), 613-636.

[4] Burgos, J. I., Arithmetic Chow rings and Deligne-Beilinson cohomology, J. Algebraic Geom., 6 (1997), 335-377.

[5] Hermitian vector bundles and characteristic classes, in The arithmetic and geometry of algebraic cycles, Amer. Math. Soc., Providence, 2000, pp. 155-182.

[6] Burgos, J. I. and Wang, S., Higher Bott-Chern forms and Beilinson's regulator, Invent. Math., 132 (1998), 261-305.

[7] Deligne, P., Le déterminant de la cohomologie, Contemp. Math., 67 (1987), 93-178.

[8] Gillet, H. and Grayson, D., The loop space of a Q-construction, Illinois J. Math., 31 (1987), 574-597.

[9] Gillet, H. and Soulé, C., Characteristic classes for algebraic vector bundles with hermitian metric, Ann. of Math., 131 (1990), 163-238.

[10] - Analytic torsion and the arithmetic Todd genus, Topology, 30 (1991), 21-54.

[11] Goerss, P. and Jardine, J., Simplicial homotopy theory, Progr. Math., 174, Birkhäuser, Basel, 1999.

[12] Künnemann, K., Arakelov Chow groups of abelian schemes, arithmetic Fourier transform, and analogues of the standard conjectures of Lefschetz type, Math. Ann., 300 (1994), 365-392.

[13] Roessler, D., Analytic torsion for cubes of vector bundles and Gillet's Riemann-Roch theorem, J. Algebraic Geom., 8 (1999), 497-518.

[14] Soulé, C., Lectures on Arakelov geometry, Cambridge Univ. Press, Cambridge, 1992.

[15] Waldhausen, F., Algebraic K-theory of spaces, in Algebraic and geometric topology, Lecture Notes in Math., Springer, Berlin, 1126, (1980), 318-419. 